This is the author's final, peer-reviewed manuscript as accepted for publication. The publisher-formatted version may be available through the publisher's web site or your institution's library.

\title{
One-dimensional inverse scattering and spectral problems
}

\author{
A.G. Ramm
}

\section{How to cite this manuscript}

If you make reference to this version of the manuscript, use the following information:

Ramm, A.G. (2004). One-dimensional inverse scattering and spectral problems.

Retrieved from http://krex.ksu.edu

\section{Published Version Information}

\section{Citation:}

Ramm, A.G. (2004). One-dimensional inverse scattering and spectral problems. CUBO, A Mathematical Journal, 6(1), 313-426.

\section{Copyright:}

Digital Object Identifier (DOI):

Publisher's Link: http://dme.ufro.cl/cubo/index.php 
A.G.Ramm, One-dimensional inverse scattering and spectral problems, CUBO a Math. Journal, 6, N1, (2004), 313-426 


\title{
One-dimensional inverse scattering and spectral problems ${ }^{12}$
}

\author{
A.G. Ramm \\ Mathematics Department, Kansas State University, \\ Manhattan, KS 66506-2602, USA \\ ramm@math.ksu.edu \\ http://www.math.ksu.edu/〜 ramm
}

${ }^{1}$ key words: property $\mathrm{C}$ for ODE, inverse spectral and scattering problems, inverse problems for PDE and ODE, spectral and scattering theory.

${ }^{2}$ Math subject classification: 35R30, 34B25, 34A55, 81F05, 81F15 


\begin{abstract}
Inverse scattering and spectral one-dimensional problems are discussed systematically in a selfcontained way. Many novel results due to the author are presented. The classical results are often presented in a new way. Several highlights of the new results include:

1) Analysis of the invertibility of the steps in the Gel'fand-Levitan and Marchenko inversion procedures,

2) Theory of the inverse problem with $I$-function as the data and its applications;

3) Proof of the property $\mathrm{C}$ for ordinary differential operators, numerous applications of property C;

4) Inverse problems with "incomplete" data;

5) Spherically symmetric inverse scattering problem with fixed-energy data: analysis of the NewtonSabatier (NS) scheme for inversion of fixed-energy phase shifts is given. This analysis shows that the NS scheme is fundamentally wrong, and is not a valid inversion method.

6) Complete presentation of the Krein inverse scattering theory is given. Consistency of this theory is proved.

7) Quarkonium systems;

8) A study of the properties of $I$-function;

9) Some new inverse problems for the heat and wave equations are studied.
\end{abstract}

10) A study of inverse scattering problem for an inhomogeneous Schrödinger equation; 


\section{Contents}

1 Introduction $\quad 3$

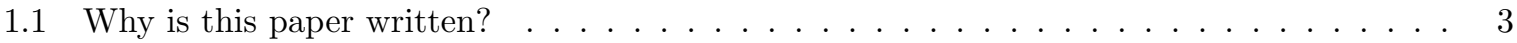

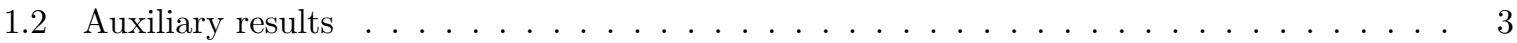

1.3 Statement of the inverse scattering and inverse spectral problems. . . . . . . . . . 7

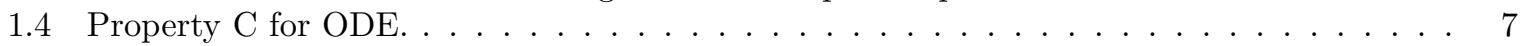

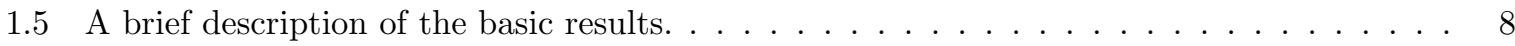

2 Property C for ODE $\quad 12$

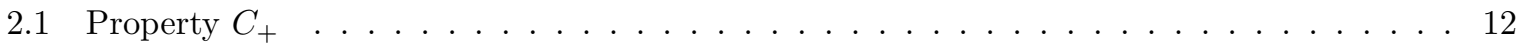

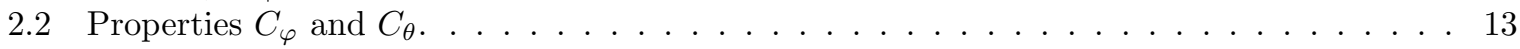

3 Inverse problem with $I$-function as the data 16

3.1 Uniqueness theorem . . . . . . . . . . . . . . . . . . . . . 16

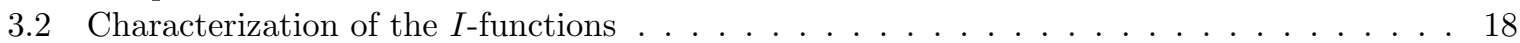

3.3 Inversion procedures. . . . . . . . . . . . . . . . . . . . . 19

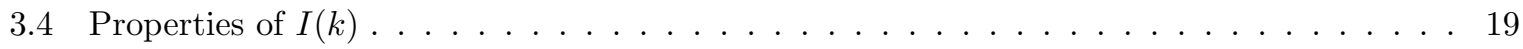

4 Inverse spectral problem $\quad \mathbf{2 7}$

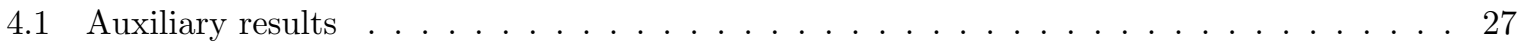

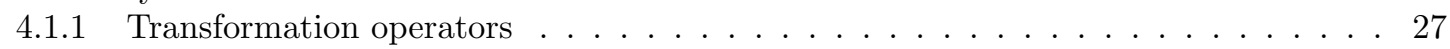

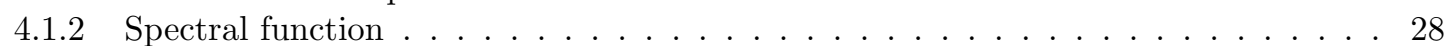

4.2 Uniqueness theorem . . . . . . . . . . . . . . . . . . . . . 29

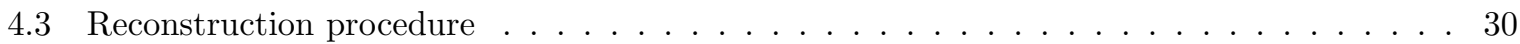

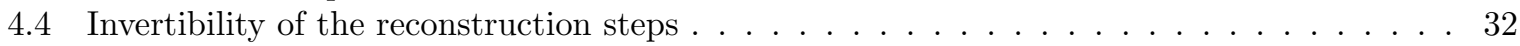

4.5 Characterization of the class of spectral functions of the Sturm-Liouville operators . . . . 33

4.6 Relation to the inverse scattering problem . . . . . . . . . . . . . . . 34

$5 \quad$ Inverse scattering on half-line $\quad 36$

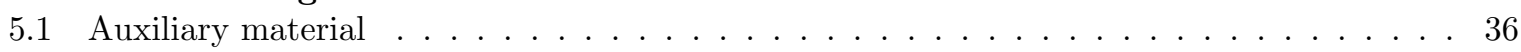

5.1 .1 Transformation operators . . . . . . . . . . . . . . . . 36

5.1.2 Statement of the direct scattering problem on half-axis. Existence and uniqueness

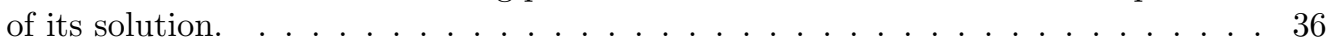

5.1 .3 Higher angular momenta. . . . . . . . . . . . . . . . . . . . 37

5.1 .4 Eigenfunction expansion . . . . . . . . . . . . . . . . . . 39

5.2 Statement of the inverse scattering problem on the half-line. Uniqueness theorem . . . . . 40

5.3 Reconstruction procedure . . . . . . . . . . . . . . . . . 41

5.4 Invertibility of the steps of the reconstruction procedure . . . . . . . . . . . . 44

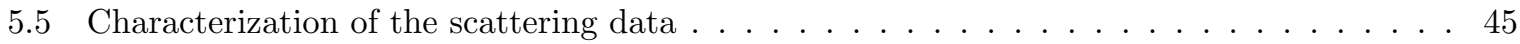

5.6 A new equation of Marchenko-type . . . . . . . . . . . . . . . . . 46

5.7 Inequalities for the transformation operators and applications . . . . . . . . . . . 47 


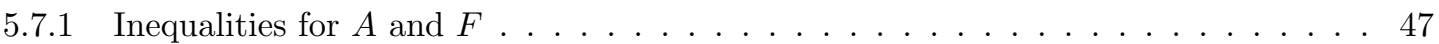

5.7 .2 Characterization of the scattering data revisited . . . . . . . . . . . . . 50

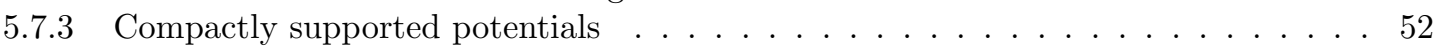

5.7 .4 Square integrable potentials . . . . . . . . . . . . . . . . . . 53

6 Inverse scattering problem with fixed-energy phase shifts as the data $\quad 54$

6.1 Introduction . . . . . . . . . . . . . . . . . . . . . 54

6.2 Existence and uniqueness of the transformation operators independent of angular momentum 55

6.3 Uniqueness theorem. . . . . . . . . . . . . . . . . . . . . . . . . . 61

6.4 Why is the Newton-Sabatier (NS) procedure fundamentally wrong? . . . . . . . . . . . . 61

6.5 Formula for the radius of the support of the potential in terms of scattering data . . . . 66

7 Inverse scattering with "incomplete data" $\quad \mathbf{7 0}$

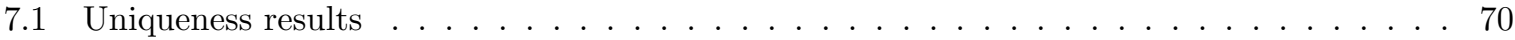

7.2 Uniqueness results: compactly supported potentials . . . . . . . . . . . . . . . 72

7.3 Inverse scattering on the full line by a potential vanishing on a half-line $\ldots \ldots \ldots \ldots$

8 Recovery of quarkonium systems $\quad \mathbf{7 4}$

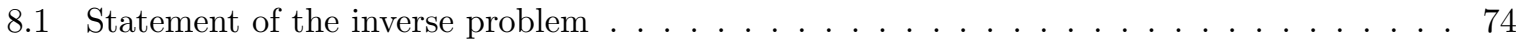

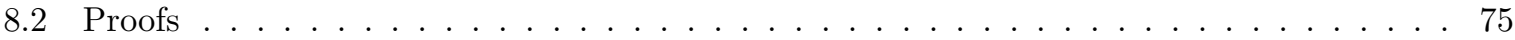

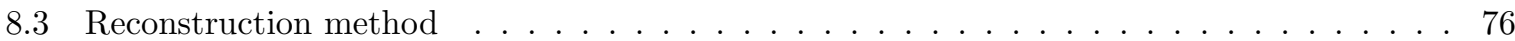

9 Krein's method in inverse scattering $\quad \mathbf{7 8}$

9.1 Introduction and description of the method $\ldots \ldots \ldots \ldots \ldots \ldots$

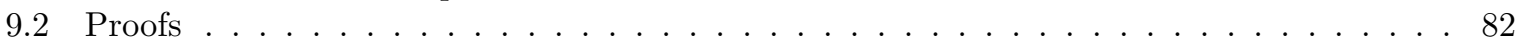

9.3 Numerical aspects of the Krein inversion procedure. . . . . . . . . . . . . . . . 88

9.4 Discussion of the ISP when the bound states are present. . . . . . . . . . . . . . 88

9.5 Relation between Krein's and GL's methods. . . . . . . . . . . . . . . . . 88

10 Inverse problems for the heat and wave equations. $\quad 90$

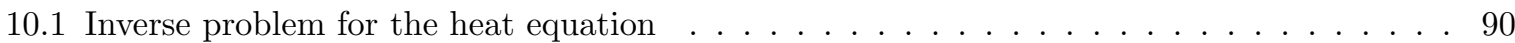

10.2 What are the "correct" measurements? . . . . . . . . . . . . . . . . . . . . 91

10.3 Inverse problem for the wave equation $\ldots \ldots \ldots \ldots \ldots \ldots \ldots \ldots \ldots \ldots \ldots$

11 Inverse problem for an inhomogeneous Schrödinger equation $\quad 93$

$\begin{array}{ll}\text { Bibliography } & 96\end{array}$ 


\section{Chapter 1}

\section{Introduction}

\subsection{Why is this paper written?}

There are excellent books $[\mathrm{M}]$ and $[\mathrm{L}]$, where inverse spectral and scattering problems are discussed in detail. The author decided to write this paper for the following reasons: 1) He gives a new approach to the uniqueness of the solutions to these problems. This approach is based on property $C$ for SturmLiouville operators; 2) the inverse problem with $I$-function as the data is studied and applied to many inverse problems; 3) a detailed analysis of the invertibility of the steps in Marchenko and Gel'fandLevitan (GL) inversion procedures is given; 4) inverse problems with "incomplete" data are studied; 5) a detailed presentation of Krein's inversion method with proofs is given apparently for the first time; 6) a number of new results for various inverse problems are presented. These include, in particular, a) analysis of the Newton-Sabatier (NS) inversion scheme for finding a potential given the corresponding fixed-energy phase shifts: it is proved that the NS scheme is fundamentally wrong and is not an inversion method; b) a method for finding confining potential (a quarkonium system) from a few experimental data; c) solution of several new inverse problems for the heat- and wave equations; d) a uniqueness theorem for finding a potential $q$ from a part of the corresponding fixed-energy phase shifts; and many other results which are taken from [R], [R1]-[R29].

Due to the space limitations, several important questions are not discussed: inverse scattering on the full line, iterative methods for finding potential $q$ : a) from two spectra [R],[R5], b) from $S$-matrix alone when $q$ is compactly supported [R9], approximate methods for finding $q$ from fixed-energy phase shifts [R14],[R15], property of resonances [R], [R29], inverse scattering for systems of equations, etc.

\subsection{Auxiliary results}

Let $q(x) \in L_{1,1}, L_{1, m}=\left\{q: q(x)=\overline{q(x)}, \int_{0}^{\infty}(1+x)^{m}|q(x)| d x<\infty\right.$, and $\left.q \in L_{\text {loc }}^{2}\left(\mathbb{R}_{+}\right)\right\}$, where $L_{\text {loc }}^{2}\left(\mathbb{R}_{+}\right)$ consists of functions belonging to $L^{2}(0, a)$ for any $a<\infty$, and overline stands for complex conjugate.

Consider the differential expression $\ell u=-u^{\prime \prime}+q(x) u$ with domain of definition $D\left(l_{0}\right)=\{u: u(0)=$ $\left.0, u \in C_{0}^{2}(0, \infty)\right\}$, where $C_{0}^{2}(0, \infty)$ is the set of $C^{2}\left(\mathbb{R}_{+}\right)$-functions vanishing in a neighborhood of infinity, $\mathbb{R}_{+}:=[0, \infty)$. If $H$ is the Hilbert space $L^{2}\left(\mathbb{R}_{+}\right)$, then $\ell_{0}$ is densely defined symmetric linear operator in $H$, essentially self-adjoint, that is, the closure $\ell$ of $\ell_{0}$ in $H$ is selfadjoint. It is possible to construct a selfadjoint operator $\ell$ without assuming that $q \in L_{l o c}^{2}\left(\mathbb{R}_{+}\right)$. Such a theory is technically more difficult, because it is not even obvious a priori that the set $D\left(\ell_{0}\right):=\left\{u: u \in C_{0}^{2}\left(\mathbb{R}_{+}\right), \ell u \in L^{2}\left(\mathbb{R}_{+}\right)\right\}$is dense in $H$ (in fact, it is dense). Such a theory is presented in [Nai]. If one drops the assumption $q \in L_{\text {loc }}^{2}$, then $D\left(\ell_{0}\right)$ is not a domain of definition of $\ell$ since there are functions $u \in \mathcal{D}\left(\ell_{0}\right)$ for which $\ell u \notin L^{2}\left(\mathbb{R}_{+}\right)$. In the future we mean by $\ell$ a self-adjoint operator generated by the differential expression $\ell$ and the boundary condition $u(0)=0$. 
This operator has absolutely continuous spectrum, which fills $(0, \infty)$, and discrete, finite, negative spectrum $\left\{-k_{j}^{2}\right\}_{1 \leq j \leq J}$, where $-k_{j}^{2}$ are the eigenvalues of $\ell$, all of them are simple,

$$
\ell \varphi_{j}:=-\varphi_{j}^{\prime \prime}+q \varphi_{j}=-k_{j}^{2} \varphi_{j}, \quad \varphi_{j}(0)=0, \quad \varphi_{j}^{\prime}(0)=1,
$$

where $\varphi_{j}$ are corresponding eigenfunctions which are real-valued functions, and

$$
\frac{1}{c_{j}}:=\int_{0}^{\infty} \varphi_{j}^{2} d x
$$

The functions $\varphi(x, k)$ and $\theta(x, k)$ are defined as the unique solutions to the problems:

$$
\begin{aligned}
& \ell \varphi=k^{2} \varphi, x>0 ; \quad \varphi(0, k)=0, \varphi^{\prime}(0, k)=1, \\
& \ell \theta=k^{2} \theta, x>0 ; \quad \theta(0, k)=1, \quad \theta^{\prime}(0, k)=0 .
\end{aligned}
$$

These functions are well defined for any $q(x) \in L_{l o c}^{1}\left(\mathbb{R}_{+}\right)$. Their existence and uniqueness can be proved by using the Volterra equations for $\varphi$ and $\theta$. If $q \in L_{1,1}$, then the Jost solution $f(x, k)$ exists and is unique. This solution is defined by the problem:

$$
\ell f:=-f^{\prime \prime}+q f=k^{2} f, f(x, k)=\exp (i k x)+o(1) \text { as } x \rightarrow+\infty ; f(0, k):=f(k) .
$$

Existence and uniqueness of $f$ is proved by means of the Volterra equation:

$$
f(x, k)=\exp (i k x)+\int_{x}^{\infty} \frac{\sin [k(t-x)]}{k} q(t) f(t, k) d t .
$$

If $q \in L_{1,1}$ then this equation implies that $f(x, k)$ is an analytic function of $k$ in $\mathbb{C}_{+}=\{k: \operatorname{Im} k>0\}$, $f(x, k)=\overline{f(x,-k)}$ for $k>0$. The Jost function is defined as $f(k):=f(0, k)$. It has exactly $J$ simple roots $i k_{j}, k_{j}>0$, where $-k_{j}^{2}, 1 \leq j \leq J$, are the negative eigenvalues of $\ell$. The number $k=0$ can be a zero of $f(k)$. If $f(0)=0$, then $\dot{f}(0) \neq 0$, where $\dot{f}(k):=\frac{d f}{d k}$. Existence of $\dot{f}(0)$ is a fine result under the only assumption $q \in L_{1,1}$ ( see Theorem 3.1.3 below, and $[\mathrm{R}]$ ) and an easy one if $q \in L_{1,2}:=\left\{q: q=\bar{q}, \int_{0}^{\infty}\left(1+x^{2}\right)|q(x)| d x<\infty\right\}$. The phase shift $\delta(k)$ is defined by the formula

$$
f(k)=|f(k)| \exp (-i \delta(k)), \quad \delta(\infty)=0, \quad f(\infty)=1,
$$

where the last equation in (1.2.18) follows from (1.2.6). Because $q(x)=\overline{q(x)}$, one has $\delta(-k)=-\delta(k)$ for $k \in \mathbb{R}$. One defines the $S$-matrix by the formula

$$
S(k):=\frac{f(-k)}{f(k)}, \quad k \in \mathbb{R} .
$$

The function $S(k)$ is not defined for complex $k$ if $q \in L_{1,1}$, but if $|q(x)| \leq c_{1} \exp \left(-c_{2}|x|^{\gamma}\right), \gamma>1$, then $f(k)$ is an entire function of $k$ and $S(k)$ is meromorphic in $\mathbb{C}$. If $q(x)=0$ for $x>a$, then $f(k)$ is an entire function of exponential type $\leq 2 a$ (see Section 5.1).

If $q \in L_{1,1}$, then at $k^{2}=-k_{j}^{2}, k_{j}>0$, the Jost solution $f_{j}(x):=f\left(x, i k_{j}\right)$ is proportional to $\varphi_{j}(x):=\varphi\left(x, i k_{j}\right), f_{j}$ and $\varphi_{j}$ both belong to $L^{2}\left(\mathbb{R}_{+}\right)$. The integral equation for $\varphi$ is:

$$
\varphi(x, k)=\frac{\sin (k x)}{k}+\int_{0}^{x} \frac{\sin k(x-s)}{k} q(s) \varphi(s, k) d s .
$$

One has:

$$
\varphi(x, k)=\frac{f(x, k) f(-k)-f(x,-k) f(k)}{2 i k},
$$


because the right-hand side of (1.2.10) solves equation (1.2.5) and satisfies conditions (1.2.3) at $x=0$. The first condition (1.2.3) is obvious, and the second one follows from the Wronskian formula:

$$
f^{\prime}(0, k) f(-k)-f^{\prime}(0,-k) f(k)=2 i k .
$$

If $k=i k_{j}$ then $f_{j}(x) \in L^{2}\left(\mathbb{R}_{+}\right)$, as one can derive easily from equation (1.2.6). In fact, $\left|f_{j}(x)\right| \leq c e^{-k_{j} x}$, $x \geq 0$. If $k>0$, then $f(x,-k)=f(x, k)$. If $q=\bar{q}$ then $f_{j}(x)$ is a real-valued function. The function $f(x, k)$ is analytic in $\mathbb{C}_{+}$but is, in general, not defined for $k \in \mathbb{C}_{-}:=\{k: \operatorname{Im} k<0\}$. In particular, (1.2.11), in general, is valid on the real axis only. However, if $|q(x)| \leq c_{1} \exp \left(-c_{2}|x|^{\gamma}\right), \gamma>1$, then $f(k)$ is defined on the whole complex plane of $k$, as was mentioned above. Let us denote $f(x, k):=f_{+}(x, k)$ for $k \in \mathbb{C}_{+}$and let $f_{-}(x, k)$ be the second, linearly independent, solution to equation (1.2.5) for $k \in \mathbb{C}_{+}$. If $f_{+} \in L^{2}\left(\mathbb{R}_{+}\right)$, then $f_{-} \notin L^{2}\left(\mathbb{R}_{+}\right)$. One can write a formula, similar to (1.2.10), for $k \in \mathbb{C}_{+}$:

$$
\varphi(x, k)=c(k)\left[f_{-}(0, k) f(x, k)-f(0, k) f_{-}(x, k)\right],
$$

where $c(k)=$ const $\neq 0$. For $\varphi\left(x, i k_{j}\right) \in L^{2}\left(\mathbb{R}_{+}\right)$, it is necessary and sufficient that $f\left(i k_{j}\right)=0$. In fact

$$
f\left(i k_{j}\right)=0, \quad \dot{f}\left(i k_{j}\right) \neq 0, \quad 1 \leq j \leq J,
$$

where $\dot{f}=\frac{d f}{d k}$. To prove the second relation in (1.2.13), one differentiates (1.2.5) with respect to $k$ and gets

$$
\dot{f}^{\prime \prime}+k^{2} \dot{f}-q \dot{f}=-2 k f .
$$

Existence of the derivative $\dot{f}$ with respect to $k$ in $\mathbb{C}_{+}$follows easily from equation (1.2.6). Multiply (1.2.14) by $f$ and (1.2.5) by $\dot{f}$, subtract and integrate over $\mathbb{R}_{+}$, then by parts, put $k=i k_{j}$, and get:

$$
-2 i k_{j} \int_{0}^{\infty} f_{j}^{2} d x=\left.\left(f \dot{f}^{\prime}-f^{\prime} \dot{f}\right)\right|_{0} ^{\infty}=f^{\prime}\left(0, i k_{j}\right) \dot{f}\left(i k_{j}\right) .
$$

Thus

$$
\int_{0}^{\infty} f_{j}^{2} d x=\frac{f^{\prime}\left(0, i k_{j}\right) \dot{f}\left(i k_{j}\right)}{-2 i k_{j}}:=\frac{1}{s_{j}}>0 .
$$

It follows from (1.2.15) that $\dot{f}\left(i k_{j}\right) \neq 0$. The numbers $s_{j}>0$ are called the norming constants:

$$
s_{j}=-\frac{2 i k_{j}}{f^{\prime}\left(0, i k_{j}\right) \dot{f}\left(i k_{j}\right)}, \quad 1 \leq j \leq J .
$$

Definition 1.2.1. Scattering data is the triple:

$$
\mathcal{S}:=\left\{S(k), k_{j}, s_{j}, 1 \leq j \leq J\right\}, \quad S(k):=\frac{f(-k)}{f(k)}, \quad k_{j}>0, \quad s_{j}>0 .
$$

The Jost function $f(k)$ may vanish at $k=0$. If $f(0)=0$, then the point $k=0$ is called a resonance. If $|q(x)| \leq c_{1} \exp \left(-c_{2}|x|^{\gamma}\right), \gamma>1$, then the zeros of $f(k)$ in $\mathbb{C}_{-}$are called resonances. As we have seen above, there are finitely many zeros of $f(k)$ in $\mathbb{C}_{+}$, these zeros are simple, their number $J$ is the number of negative eigenvalues $-k_{j}^{2}, 1 \leq j \leq J$, of the selfadjoint Dirichlet operator $\ell$. If $q \in L_{1,1}$ then the negative spectrum of $\ell$ is finite $[\mathrm{M}]$.

The phase shift $\delta(k)$, defined in (1.2.18), is related to $S(k)$ :

$$
S(k)=e^{2 i \delta(k)},
$$

so that $S(k)$ and $\delta(k)$ are interchangeable in the scattering data. One has $f_{j}(x)=f^{\prime}\left(0, i k_{j}\right) \varphi_{j}(x)$, because $\frac{f\left(x, i k_{j}\right)}{f^{\prime}\left(0, i k_{j}\right)}$ solves (1.2.3). Therefore

$$
\int_{0}^{\infty} \varphi_{j}^{2} d x=\frac{1}{s_{j}\left[f^{\prime}\left(0, i k_{j}\right)\right]^{2}}:=\frac{1}{c_{j}} .
$$


Thus

$$
c_{j}=-\frac{2 i k_{j} f^{\prime}\left(0, i k_{j}\right)}{\dot{f}\left(i k_{j}\right)}, \quad 1 \leq j \leq J
$$

In Section 4.1 the notion of spectral function $\rho(\lambda)$ is defined. It will be proved in Section 5.1 for $q \in L_{1,1}$ that the formula for the spectral function is:

$$
d \rho(\lambda)=\left\{\begin{array}{cc}
\frac{\sqrt{\lambda} d \lambda}{\pi|f(\sqrt{\lambda})|^{2}}, \quad \lambda \geq 0, \\
\sum_{j=1}^{J} c_{j} \delta\left(\lambda+k_{j}^{2}\right) d \lambda, & \lambda<0,
\end{array}\right.
$$

where $c_{j}$ are defined in (1.2.19)-(1.2.20). The spectral function is defined in Section 4.1 for any $q \in$ $L_{\text {loc }}^{1}\left(\mathbb{R}_{+}\right), q=\bar{q}$. Such a $q$ may grow at infinity. On the other hand, the scattering theory is constructed for $q \in L_{1,1}$.

Let us define the index of $S(k)$ :

$$
\mathcal{J}:=\operatorname{ind} S(k):=\frac{1}{2 \pi} \Delta_{\mathbb{R}} \arg S(k)=\frac{1}{2 \pi i} \int_{-\infty}^{\infty} d \ln S(k) .
$$

This definition implies that ind $S(k)=$ ind $f(-k)-$ ind $f(k)=-2$ ind $f(k)$. Therefore:

$$
\text { ind } S(k)=\left\{\begin{aligned}
-2 J & \text { if } f(0) \neq 0 \\
-2 J-1 & \text { if } f(0)=0
\end{aligned}\right.
$$

because a simple zero $k=0$ contributes $\frac{1}{2}$ to the index, and the index of an analytic in $\mathbb{C}_{+}$function $f(k)$, such that $f(\infty)=1$, equals to the number of zeros of $f(k)$ in $\mathbb{C}_{+}$plus half of the number of its zeros on the real axis, provided that all the zeros are simple. This follows from the argument principle.

In Section 4.2 and Section 5.2 the existence and uniqueness of the transformation (transmutation) operators will be proved. Namely,

$$
\varphi(x, k)=\varphi_{0}(x, k)+\int_{0}^{x} K(x, y) \varphi_{0}(y) d y:=(I+K) \varphi_{0}, \quad \varphi_{0}:=\frac{\sin (k x)}{k}
$$

and

$$
f(x, k)=e^{i k x}+\int_{x}^{\infty} A(x, y) e^{i k y} d y:=(I+A) f_{0}, \quad f_{0}:=e^{i k x},
$$

and the properties of the kernels $A(x, y)$ and $K(x, y)$ are discussed in Section 5.2 and Section 4.2 correspondingly. The transformation operator $I+K$ transforms the solution $\varphi_{0}$ to the equation (1.2.3) with $q=0$ into the solution $\varphi$ of (1.2.3), satisfying the same as $\varphi_{0}$ boundary conditions at $x=0$. The transformation operator $I+A$ transforms the solution $f_{0}$ to equation (1.2.5) with $q=0$ into the solution $f$ of (1.2.5) satisfying the same as $f_{0}$ "boundary conditions at infinity".

One can prove (see $[\mathrm{M}]$ and Sec. 5.7) the following estimates

$$
\begin{array}{r}
|A(x, y)| \leq c \sigma\left(\frac{x+y}{2}\right), \quad c=\text { const }>0, \quad \sigma(x):=\int_{x}^{\infty}|q(t)| d t, \\
\left|A_{y}(x, y)+\frac{1}{4} q\left(\frac{x+y}{2}\right)\right|+\left|A_{x}(x, y)+\frac{1}{4} q\left(\frac{x+y}{2}\right)\right| \leq c \sigma(x) \sigma\left(\frac{x+y}{2}\right),
\end{array}
$$

and $A(x, y)$ solves the equation:

$$
A(x, y)=\frac{1}{2} \int_{\frac{x+y}{2}}^{\infty} q d s+\int_{\frac{x+y}{2}}^{\infty} d s \int_{0}^{\frac{y-x}{2}} d t q(s-t) A(s-t, s+t) .
$$

By $H^{m}=H^{m}\left(\mathbb{R}_{+}\right)$we denote Sobolev spaces $W^{m, 2}$. The kernel $A(x, y)$ is the unique solution to (1.2.28), and also of the problem (5.1.1)-(5.1.3). 


\subsection{Statement of the inverse scattering and inverse spectral problems.}

ISP: Inverse Scattering problem (ISP) consists of finding $q \in L_{1,1}$ from the corresponding scattering data $\mathcal{S}$ (see (1.2.6)).

A study of ISP consists of the following:

1) One proves that ISP has at most one solution (see Theorem 5.2.1).

2) One finds necessary and sufficient conditions for $\mathcal{S}$ to be scattering data corresponding to a $q \in L_{1,1}$ (characterization of the scattering data problem).

3) One gives a reconstruction method for calculating $q \in L_{1,1}$ from the corresponding $\mathcal{S}$.

In Chapter 5 these three problems are solved.

ISpP: Inverse spectral problem consists of finding $q$ from the corresponding spectral function.

A study of ISpP consists of the similar steps:

1) One proves that ISpP has at most one solution in an appropriate class of $q$ : if $q_{1}$ and $q_{2}$ from this class generate the same $\rho(\lambda)$, then $q_{1}=q_{2}$.

2) One finds necessary and sufficient conditions on $\rho(\lambda)$ which guarantee that $\rho(\lambda)$ is a spectral function corresponding to some $q$ from the above class.

3) One gives a reconstruction method for finding $q(x)$ from the corresponding $\rho(\lambda)$.

\subsection{Property $\mathrm{C}$ for ODE.}

Denote by $\ell_{m}$ operators $\ell$ corresponding to potentials $q_{m} \in L_{1,1}$, and by $f_{m}(x, k)$ the corresponding Jost solutions, $m=1,2$.

Definition 1.4.1. We say that a pair $\left\{\ell_{1}, \ell_{2}\right\}$ has property $C_{+}$iff the set $\left\{f_{1}(x, k) f_{2}(x, k)\right\}_{\forall k>0}$ is complete (total) in $L^{1}\left(\mathbb{R}_{+}\right)$.

This means that if $h \in L^{1}\left(\mathbb{R}_{+}\right)$then

$$
\left\{\int_{0}^{\infty} h(x) f_{1}(x, k) f_{2}(x, k) d x=0 \quad \forall k>0\right\} \Rightarrow h=0 .
$$

We prove in Section 2.1 that a pair $\left\{\ell_{1}, \ell_{2}\right\}$ does have property $C_{+}$if $q_{m} \in L_{1,1}$. Let $\ell \varphi:=-\varphi^{\prime \prime}+$ $q(x) \varphi$, and let $\varphi_{j}$ correspond to $q=q_{j}$,

$$
\ell \varphi-k^{2} \varphi=0, \quad \varphi(0, k)=0 ; \quad \varphi^{\prime}(0, k)=1 ; \quad \ell \theta-k^{2} \theta=0, \quad \theta(0, k)=1, \quad \theta^{\prime}(0, k)=0 .
$$

Definition 1.4.2. We say that a pair $\left\{\ell_{1}, \ell_{2}\right\}$ has property $C_{\varphi}$ iff the set $\left\{\varphi_{1}(\cdot, k) \varphi_{2}(\cdot, k)\right\}$ is complete in $L^{1}(0, b)$ for any $b>0, b<\infty$.

This means that if $h \in L^{1}(0, b)$, then:

$$
\left\{\int_{0}^{b} h(x) \varphi_{1}(x, k) \varphi_{2}(x, k) d x\right\}_{\forall k>0} \Rightarrow h=0 .
$$

In Theorem 2.2.2 we prove that there is a $h \neq 0$ for which

$$
\int_{0}^{\infty} h(x) \varphi_{1}(x, k) \varphi_{2}(x, k) d x=0 \quad \forall k>0
$$


for a suitable $q_{1} \neq q_{2}, q_{1}, q_{2} \in L_{1,1}$. Therefore Property $C_{\varphi}$ with $b=\infty$ does not hold, in general.

Property $C_{\theta}$ is defined similarly to Property $C_{\varphi}$, with functions $\theta_{j}(x, k)$ replacing $\varphi_{j}(x, k)$.

In Chapter 2 we prove that properties $C_{+}, C_{\varphi}$ and $C_{\theta}$ hold, and give many applications of these properties throughout this work.

\subsection{A brief description of the basic results.}

The basic results of this work include:

1) Proof of properties $C_{+}, C_{\varphi}$ and $C_{\theta}$. Demonstration of many applications of these properties.

2) Analysis of the invertibility of the steps in the inversion procedures of Gel'fand-Levitan (GL) for solving inverse spectral problem:

$$
\rho \Rightarrow L \Rightarrow K \Rightarrow q
$$

where

$$
q=2 \frac{d K(x, x)}{d x}
$$

the kernel $L=L(x, y)$ is:

$$
\begin{gathered}
L(x, y)=\int_{-\infty}^{\infty} \varphi_{0}(x, \lambda) \varphi_{0}(y, \lambda) d \sigma(\lambda), \quad d \sigma(\lambda):=d\left[\rho(\lambda)-\rho_{0}(\lambda)\right], \\
d \rho_{0}=\left\{\begin{array}{cl}
\frac{\sqrt{\lambda} d \lambda}{\pi}, & \lambda \geq 0, \\
0, & \lambda<0 .
\end{array}\right.
\end{gathered}
$$

$\rho_{0}=\frac{2 \lambda^{3 / 2}}{3 \pi}, \rho_{0}$ is the spectral function of $\ell$ with $q=0$, and $K$ solves the Gel'fand-Levitan equation

$$
K(x, y)+\int_{0}^{x} K(x, s) L(s, y) d s+L(x, y)=0, \quad 0 \leq y \leq x .
$$

Our basic result is a proof of the invertibility of all the steps in (1.5.1):

$$
\rho \Leftrightarrow L \Leftrightarrow K \Leftrightarrow q,
$$

which holds under a weak assumption on $\rho$. Namely, assume that

$$
\rho \in \mathcal{G},
$$

where $\mathcal{G}$ is the set of nondecreasing functions $\rho$ of bounded variation on every interval $(-\infty, b), b<\infty$, such that the following two assumptions, $\left.A_{1}\right)$ and $A_{2}$ ) hold.

Denote $L_{0}^{2}\left(\mathbb{R}_{+}\right)$the set of $L^{2}\left(\mathbb{R}_{+}\right)$functions vanishing in a neighborhood of infinity. Let $h \in L_{0}^{2}\left(\mathbb{R}_{+}\right)$ and $H(\lambda):=\int_{0}^{\infty} h(x) \varphi_{0}(x, \lambda) d x$.

Assumption $\left.A_{1}\right)$ is:

$$
\text { If } h \in L_{0}^{2}\left(\mathbb{R}_{+}\right) \text {and } \int_{-\infty}^{\infty} H^{2}(\lambda) d \rho(\lambda)=0 \text {, then } h=0 .
$$

Let

$$
\mathcal{H}:=\left\{H(\lambda): h \in C_{0}^{\infty}\left(\mathbb{R}_{+}\right)\right\}, \quad H(\lambda):=\int_{0}^{\infty} h(x) \varphi_{0}(x, \lambda) d x
$$


$\rho_{1}$ and $\rho_{2}$ belong to $\mathcal{P}$, and $\nu:=\rho_{1}-\rho_{2}$ (see Section 4.2).

Assumption $A_{2}$ ) is:

$$
\text { If } \int_{-\infty}^{\infty} H^{2}(\lambda) d \nu=0 \quad \forall H \in \mathcal{H} \text {, then } \nu=0 .
$$

In order to insure the one-to-one correspondence between spectral functions $\rho$ and selfadjoint operators $\ell$, we assume that $q$ is such that the corresponding $\ell$ is "in the limit point at infinity case". This means that the equation $(\ell-z) u=0, \operatorname{Im} z>0$ has exactly one nontrivial solution in $L^{2}\left(\mathbb{R}_{+}\right)$, $\ell u=-u^{\prime \prime}+q(x) u$. If $q \in L_{1,1}$ then $\ell$ is "in the limit point at infinity case".

3) Analysis of the invertibility of the Marchenko inversion procedure for solving ISP:

$$
\mathcal{S} \Rightarrow F \Rightarrow A \Rightarrow q,
$$

where

$$
\begin{gathered}
F(x):=\frac{1}{2 \pi} \int_{-\infty}^{\infty}[1-S(k)] e^{i k x} d x+\sum_{j=1}^{J} s_{j} e^{-k_{j} x}:=F_{s}(x)+F_{d}(x), \\
q(x)=-2 \frac{d A(x, x)}{d x},
\end{gathered}
$$

and $A(x, y)$ solves the Marchenko equation

$$
A(x, y)+\int_{x}^{\infty} A(x, s) F(s+y) d s+F(x+y)=0, \quad 0 \leq x \leq y<\infty .
$$

Our basic result is a proof of the invertibility of the steps in (1.5.10):

$$
\mathcal{S} \Leftrightarrow F \Leftrightarrow A \Leftrightarrow q
$$

under the assumption $q \in L_{1,1}$. We also derive a new equation for

$$
A(y):=\left\{\begin{aligned}
A(0, y), & y \geq 0 \\
0, & y<0 .
\end{aligned}\right.
$$

This equation is:

$$
F(y)+A(y)+\int_{0}^{\infty} A(s) F(s+y) d s=A(-y), \quad-\infty<y<\infty .
$$

The function $A(y)$ is of interest because

$$
f(k)=1+\int_{0}^{\infty} A(y) e^{i k y} d y:=1+\widetilde{A}(k) .
$$

Therefore the knowledge of $A(y)$ is equivalent to the knowledge of $f(k)$.

In Section 5.5 we give necessary and sufficient conditions for $\mathcal{S}$ to be the scattering data corresponding to $q \in L_{1,1}$. We also prove that if

$$
|q(x)| \leq c_{1} \exp \left(-c_{2}|x|^{\gamma}\right), \quad \gamma>1,
$$

and, in particular, if

$$
q(x)=0 \text { for } x>a,
$$

then $S(k)$ alone determines $q(x)$ uniquely, because it determines $k_{j}, s_{j}$ and $J$ uniquely under the assumption (1.5.17) or (1.5.18). 
4) We give a very short and simple proof of the uniqueness theorem which says that the $I$-function,

$$
I(k):=\frac{f^{\prime}(0, k)}{f(k)}, \quad \forall k>0,
$$

determines $q \in L_{1,1}$ uniquely. The $I$-function is equal to Weyl's $m$-function if $q \in L_{1,1}$.

We give many applications of the above uniqueness theorem. In particular, we give short and simple proofs of the uniqueness theorems of Marchenko which say that $\mathcal{S}$ determines $q \in L_{1,1}$ uniquely, and $\rho(\lambda)$ determines $q$ uniquely. We prove that if (1.5.18) (or (1.5.17)) holds, then either of the four functions $S(k), \delta(k), f(k), f^{\prime}(0, k)$, determines $q(x)$ uniquely. This result is applied in Chapter 10 to the heat and wave equations. It allows one to study some new inverse problems. For example, let

$$
\begin{gathered}
u_{t t}=u_{x x}-q(x) u, \quad x>0, \quad t>0, \\
u=u_{t}=0 \text { at } t=0 . \\
u(0, t)=\delta(t) \text { or } u^{\prime}(0, t)=\delta(t) .
\end{gathered}
$$

Assume

$$
q=0 \text { for } x>1, \quad q=\bar{q}, \quad q \in L^{1}(0,1),
$$

and let the extra data (measured data) be

$$
u(1, t)=a(t) \quad \forall t>0 .
$$

The inverse problem is: given these data, find $q(x)$.

Another example: Let

$$
\begin{gathered}
u_{t}=u_{x x}-q(x) u, \quad 0 \leq x \leq 1, \quad t>0, \quad q \in L^{1}[0,1], \\
u(x, 0)=0 \\
u(0, t)=0, \quad u(1, t)=a(t), \quad a(t) \in L^{1}\left(\mathbb{R}_{+}\right), \quad a \neq 0 .
\end{gathered}
$$

The extra data are

$$
u_{x}(1, t)=b(t) \quad \forall t>0 .
$$

The inverse problem is: given these data, find $q(x)$.

Using the above uniqueness results, we prove that these two inverse problems have at most one solution. The proof gives also a constructive procedure for finding $q$.

5) We have already mentioned uniqueness theorems for some inverse problems with "incomplete data". "Incomplete data" means the data which are a proper subset of the classical data, but "incompleteness" of the data is compensated by the additional assumptions on $q$. For example, the classical scattering data are the triple (1.2.17), but if (1.5.18) or (1.5.17) is assumed, then the "incomplete data" alone, such as $S(k)$, or $\delta(k)$, or $f(k)$, or $f^{\prime}(0, k), \forall k>0$, determine $q$ uniquely. Another general result of this nature, that we prove in Chapter 7 , is the following one.

Consider, for example, the problem

$$
\ell \varphi_{j}=\lambda_{j} \varphi_{j}, \quad 0 \leq x \leq 1 ; \quad \varphi_{j}(0)=\varphi_{j}(1)=0 .
$$

Other boundary conditions can also be considered.

Assume that the following data are given.

$$
\left\{\lambda_{m(j)} \forall j ; q(x), b \leq x \leq 1\right\}, \quad q(x) \in L^{1}[0,1], q=\bar{q},
$$


where $0<b<1$, and

$$
m(j)=\frac{j}{\sigma}\left(1+\epsilon_{j}\right),\left|\epsilon_{j}\right|<1, \epsilon_{j} \rightarrow 0 \text { as } j \rightarrow \infty, \sigma=\text { const }, 0<\sigma \leq 2 .
$$

Assume also

$$
\sum_{j=1}^{\infty}\left|\epsilon_{j}\right|<\infty
$$

We prove

Theorem 1.5.1. Data (1.5.30)-(1.5.31) determine uniquely $q(x)$ on the interval $0 \leq x \leq b$ if $\sigma>2 b$. If (1.5.32) is assumed additionally, then $q$ is uniquely determined if $\sigma \geq 2 b$.

The $\sigma$ gives the "part of the spectra" sufficient for the unique recovery of $q$ on $[0, b]$. For example, if $b=\frac{1}{2}$ and (1.5.32) holds, then $\sigma=1$, so "one spectrum" determines uniquely $q$ on $\left[0, \frac{1}{2}\right]$. If $b=\frac{1}{4}$, then $\sigma=\frac{1}{2}$, so "half of the spectrum" determines uniquely $q$ on $\left[0, \frac{1}{4}\right]$. If $b=\frac{1}{5}$, then " $\frac{2}{5}$ of the spectrum" determine uniquely $q$ on $\left[0, \frac{1}{5}\right]$. If $b=1$, then $\sigma=2$, and "two spectra" determine $q$ uniquely on the whole interval $[0,1]$. The last result belongs to Borg $[\mathrm{B}]$. By "two spectra" one means $\left\{\lambda_{j}\right\} \bigcup\left\{\mu_{j}\right\}$, where $\mu_{j}$ are the eigenvalues of the problem:

$$
\ell u_{j}=\mu_{j} u_{j}, \quad u_{j}(0)=0, \quad u_{j}^{\prime}(1)+h u_{j}(1)=0 .
$$

In fact, two spectra determine not only $q$ but the boundary conditions as well $[\mathrm{M}]$.

6) Our basic results on the spherically symmetric inverse scattering problem with fixed-energy data are the following.

The first result: If $q=q(r)=0$ for $r>a, a>0$ is an arbitrary large fixed number, $r:=|x|, x \in \mathbb{R}^{3}$, $q=\bar{q}$, and $\int_{0}^{a} r^{2}|q(r)|^{2} d r<\infty$, then the data $\left\{\delta_{\ell}\right\}_{\forall \ell \in \mathcal{L}}$ determine $q(r)$ uniquely. Here $\delta_{\ell}$ is the phase shift at a fixed energy $k^{2}>0, \ell$ is the angular momentum, and $\mathcal{L}$ is any fixed set of positive integers such that

$$
\sum_{\ell \in \mathcal{L}} \frac{1}{\ell}=\infty
$$

The second result is: If $q=q(x), x \in \mathbb{R}^{3}, q=0$ for $|x|>a, q \in L^{2}\left(B_{a}\right)$, where $B_{a}:=\{x:|x| \leq a\}$, then the knowledge of the scattering amplitude $A\left(\alpha^{\prime}, \alpha\right)$ at a fixed energy $k^{2}>0$ and all $\alpha^{\prime} \in \widetilde{S}_{j}^{2}$ determine $q(x)$ uniquely [R], [R7]. Here $\widetilde{S}_{j}^{2}, j=1,2$, are arbitrary small open subsets in $S^{2}$ and $S^{2}$ is the unit sphere in $\mathbb{R}^{3}$. The scattering amplitude is defined in Section 6.1.

The third result is: The Newton-Sabatier inversion procedure (see [CS], [N]) is fundamentally wrong.

7) Following [R16] we present, apparently for the first time, a detailed exposition (with proofs) of the Krein inversion theory for solving inverse scattering problem and prove the consistency of this theory.

8) We give a method for recovery of a quarkonium system (a confining potential) from a few experimental measurements.

9) We study various properties of the $I$-function.

10) We study an inverse scattering problem for inhomogeneous Schrödinger equation. 


\section{Chapter 2}

\section{Property C for ODE}

\subsection{Property $C_{+}$}

By ODE in this section, the equation

$$
\left(\ell-k^{2}\right) u:=-u^{\prime \prime}+q(x) u-k^{2} u=0
$$

is meant. Assume $q \in L_{1,1}$. Then the Jost solution $f(x, k)$ is uniquely defined. In Section 1.4 Definition 1.4.1, property $C_{+}$is explained. Let us prove

Theorem 2.1.1. If $q \in L_{1,1}, j=1,2$ then property $C_{+}$holds.

Proof. We use (1.2.25) and (1.2.26). Denote $A(x, y):=A_{1}(x, y)+A_{2}(x, s)$. Let

$$
\begin{aligned}
0= & \int_{0}^{\infty} d x h(x) f_{1}(x, k) f_{2}(x, k) \\
= & \int_{0}^{\infty} d x h(x)\left[e^{2 i k x}+\int_{x}^{\infty} A(x, y) e^{i k y} d y\right. \\
& \left.+\int_{x}^{\infty} \int_{x}^{\infty} d y d z A_{1}(x, y) A_{2}(x, z) e^{i k(y+z)}\right]
\end{aligned}
$$

for some $h \in L^{1}\left(\mathbb{R}_{+}\right)$. Set $y+z=s, y-z=\sigma$ and get

$$
\int_{x}^{\infty} \int_{x}^{\infty} A_{1}(x, y) A_{2}(x, z) e^{i k(y+z)} d y d z=\int_{2 x}^{\infty} T(x, s) e^{i k s} d s,
$$

where

$$
T(x, s)=\frac{1}{2} \int_{-(s-2 x)}^{s-2 x} A_{1}\left(x, \frac{s+\sigma}{2}\right) A_{2}\left(x, \frac{s-\sigma}{2}\right) d \sigma .
$$

Thus, $f_{1} f_{2}=\left(I+V^{*}\right) e^{2 i k x}$, where $V^{*}$ is the adjoint to a Volterra operator, $V^{*} f:=2 \int_{x / 2}^{\infty} A(x, 2 s) f(s) d s+$ $2 \int_{x}^{\infty} T(x, 2 s) f(s) d s$.

Using (2.1.3) and (2.1.4) one rewrites (2.1.2) as

$$
0=\int_{0}^{\infty} d s e^{2 i k s}\left[h(s)+2 \int_{0}^{s} A(x, 2 s-x) h(x) d x+2 \int_{0}^{s} T(x, 2 s) h(x) d x\right], \forall k \quad>\quad 0 .
$$

The right-hand side is an analytic function of $k$ in $C_{+}$vanishing for all $k>0$. Thus, it vanishes identically in $C_{+}$and, consequently, for $k<0$. Therefore

$$
h(s)+2 \int_{0}^{s} A(x, 2 s-x) h(x) d x+2 \int_{0}^{s} T(x, 2 s) h(x) d x=0, \forall s>0 .
$$


Since $A(x, y)$ and $T(x, y)$ are bounded continuous functions, the Volterra equation (2.1.6) has only the trivial solution $h=0$.

Define functions $g_{ \pm}$and $f_{ \pm}$as the solutions to equation (1.2.5) with the following asymptotics:

$$
\begin{aligned}
& g_{ \pm}=\exp ( \pm i k x)+o(1), \quad x \rightarrow-\infty, \\
& f_{ \pm}=\exp ( \pm i k x)+o(1), \quad x \rightarrow+\infty,
\end{aligned}
$$

Let us denote $f_{+}=f$ and $g_{+}=g$.

Definition 2.1.2. The pair $\left\{\ell_{1}, \ell_{2}\right\}$ has property $C_{-}$iff the set $\left\{g_{1} g_{2}\right\}_{\forall k>0}$ is complete in $L^{1}\left(\mathbb{R}_{-}\right)$.

Similar definition can be given with $\left(g_{-, j}\right)$ replacing $g_{j}, j=1,2$.

As above, one proves:

Theorem 2.1.3. If $q_{j} \in L_{1,1}\left(\mathbb{R}_{-}\right), j=1,2$, then property $C_{-}$holds for $\left\{\ell_{1}, \ell_{2}\right\}$.

By $L_{1,1}(\mathbb{R})$ we mean the set

$$
L_{1,1}(\mathbb{R}):=\left\{q: q=\bar{q}, \int_{-\infty}^{\infty}(1+|x|)|q(x)| d x<\infty\right\} .
$$

\subsection{Properties $C_{\varphi}$ and $C_{\theta}$.}

We prove only property $C_{\varphi}$. Property $C_{\theta}$ is proved similarly. Property $C_{\varphi}$ is defined in Section 1.4.

Theorem 2.2.1. If $q_{j} \in L_{1,1}, j=1,2$, then property $C_{\varphi}$ holds for $\left\{\ell_{1}, \ell_{2}\right\}$.

Proof. Our proof is similar to the proof of Theorem 2.1.1. Using (1.2.24) and denoting $\phi=k \varphi, K:=$ $K_{1}+K_{2}$, one writes

$$
\begin{aligned}
\phi_{1} \phi_{2}= & \sin ^{2}(k x)+\int_{0}^{x} K(x, y) \sin (k x) \sin (k y) d y \\
& +\frac{1}{2} \int_{0}^{x} \int_{0}^{x} K_{1}(x, y) K_{2}(x, s)\{\cos [k(y-s)]-\cos [k(y+s)]\} d y d s .
\end{aligned}
$$

Assume:

$$
0=\int_{0}^{b} h(x) \phi_{1}(x, k) \phi_{2}(x, k) d x \quad \forall k>0
$$

Then

$$
\begin{aligned}
0 & =\int_{0}^{b} d x h(x)-\int_{0}^{b} d x h(x) \cos (2 k x) \\
& +\int_{0}^{b} d s \cos (k s) \int_{s}^{b} d x h(x) K(x, x-s) \\
& -\int_{0}^{2 b} d s \cos (k s) \int_{\frac{s}{2}}^{\min (b, s)} d x h(x) K(x, s-x)+I,
\end{aligned}
$$

where

$$
I:=\int_{0}^{b} d x h(x) \int_{0}^{x} \int_{0}^{x} K_{1}(x, y) K_{2}(x, s)\{\cos [k(y-s)]-\cos [k(y+s)]\} d y d s .
$$

Let $y-s:=t, y+s:=v$. Then

$$
\int_{0}^{x} \int_{0}^{x} K_{1} K_{2} \cos [k(y-s)] d y d s=\int_{0}^{x} d s \cos (k s) B_{1}(x, s),
$$


where

$$
\begin{gathered}
B_{1}(x, s):=\quad \frac{1}{2} \int_{|s|}^{2 x-|s|}\left[K_{1}\left(x, \frac{s+v}{2}\right) K_{2}\left(x, \frac{v-s}{2}\right)+K_{1}\left(x, \frac{v-s}{2}\right) K_{2}\left(x, \frac{v+s}{2}\right)\right] d v, \\
\int_{0}^{x} \int_{0}^{x} K_{1} K_{2} \cos [k(y+s)] d y d s=\int_{0}^{2 x} B_{2}(x, s) \cos (k s) d s, \\
B_{2}(x, s):=\frac{1}{2} \int_{-\omega(s)}^{\omega(s)} K_{1}\left(x, \frac{t+s}{2}\right) K_{2}\left(x, \frac{s-t}{2}\right) d t,
\end{gathered}
$$

and $\omega=s$ if $0 \leq s \leq x ; \omega=2 x-s$ if $x \leq s \leq 2 x$.

Therefore

$$
I=\int_{0}^{b} d s \cos (k s) \int_{s}^{b} d x h(x) B_{1}(x, s)-\int_{0}^{2 b} d s \cos (k s) \int_{\frac{s}{2}}^{b} d x h(x) B_{2}(x, s) .
$$

From (2.2.3) and (2.2.4), taking $k \rightarrow \infty$, one gets:

$$
\int_{0}^{b} h(x) d x=0
$$

and (using completeness of the system $\cos (k s), 0<k<\infty$, in $L^{2}(0, b)$ ) the following equation:

$$
\begin{gathered}
0=-\frac{h\left(\frac{s}{2}\right)}{2}+\int_{s}^{b} K(x, x-s) h(x) d x-\int_{s / 2}^{\min (b, s)} d x h(x) K(x, s-x) \\
+\int_{s}^{b} d x h(x) B_{1}(x, s)-\int_{s / 2}^{b} d x h(x) B_{2}(x, s) .
\end{gathered}
$$

The kernels $K, B_{1}$, and $B_{2}$ are bounded and continuous functions. Therefore, if $b<\infty$ and $h(x)=0$ for $x>b,(2.2 .5)$ implies:

$$
|h(y)| \leq c \int_{2 y}^{b}|h(x)| d x+c \int_{y}^{b}|h(x)| d x,
$$

where $c>0$ is a constant which bounds the kernels $2 K, 2 B_{1}$ and $2 B_{2}$ from above and $2 y=s$. From the above inequality one gets

$$
\max _{b-\epsilon \leq y \leq b}|h(y)| \leq c \epsilon \max _{b-\epsilon \leq y \leq b}|h(y)|,
$$

where $\epsilon, 0<\epsilon<b$, is sufficiently small so that $c \epsilon<1$ and $b-\epsilon<2 b-2 \epsilon$. Then inequality (2.2.6) implies $h(x)=0$ if $b-\epsilon<x<b$. Repeating this argument, one proves, in finitely many steps, that $h(x)=0$, $0<x<b$.

Theorem 2.2.1 is proved.

The proof of Theorem 2.2.1 is not valid if $b=\infty$. The result is not valid either if $b=\infty$. Let us give a counterexample.

Theorem 2.2.2. There exist $q_{1}, q_{2} \in L_{1,1}$ and an $h \neq 0$, such that

$$
\int_{0}^{\infty} h(x) \varphi_{1}(x, k) \varphi_{2}(x, k) d x=0 \quad \forall k>0 .
$$


Proof. Let $q_{1}$ and $q_{2}$ are two potenetials in $L_{1,1}$ such that $S_{1}(k)=S_{2}(k) \forall k>0, \ell_{1}$ and $\ell_{2}$ have one negative eigenvalue $-k_{1}^{2}$, which is the same for $\ell_{1}$ and $\ell_{2}$, but $s_{1} \neq s_{2}$, so that $q_{1} \neq q_{2}$. Let $h:=q_{2}-q_{1}$. Let us prove that (2.2.7) holds. One has $\ell_{1} \varphi_{1}=k^{2} \varphi_{1}, \ell_{2} \varphi_{2}=k^{2} \varphi_{2}$. subtract from the first equation the second and get:

$$
-\varphi^{\prime \prime}-k^{2} \varphi+q_{1} \varphi=h \varphi_{2}, \quad \varphi:=\varphi_{1}-\varphi_{2}, \quad \varphi(0, k)=\varphi^{\prime}(0, k)=0 .
$$

Multiply $(2.2 .8)$ by $\varphi_{1}$, integrate over $(0, \infty)$ and then by parts to get

$$
\int_{0}^{\infty} h \varphi_{2} \varphi_{1} d x=\left.\left(\varphi \varphi_{1}^{\prime}-\varphi^{\prime} \varphi_{1}\right)\right|_{0} ^{\infty}=0, \quad \forall k>0 .
$$

At $x=0$ we use conditions (2.2.8), and at $x=\infty$ the phase shifts corresponding to $q_{1}$ and $q_{2}$ are the same (because $S_{1}(k)=S_{2}(k)$ ) and therefore the right-hand side of (2.2.9) vanishes. Theorem 2.2.2 is proved. 


\section{Chapter 3}

\section{Inverse problem with $I$-function as the data}

\subsection{Uniqueness theorem}

Consider equation (1.2.5) and assume $q \in L_{1,1}$ Then $f(x, k)$ is analytic in $\mathbb{C}_{+}$. Define the $I$-function:

$$
I(k)=\frac{f^{\prime}(0, k)}{f(k)} .
$$

From (3.1.1) it follows that $I(k)$ is meromorphic in $\mathbb{C}_{+}$with the finitely many simple poles $i k_{j}, 1 \leq j \leq J$. Indeed, $i k_{j}$ are simple zeros of $f(k)$ and $f^{\prime}\left(0, i k_{j}\right) \neq 0$ as follows from (1.2.4). Using (1.2.20), one gets

$$
a_{j}:=\operatorname{Res}_{k=i k_{j}} I(k)=\frac{f^{\prime}\left(0, i k_{j}\right)}{\dot{f}\left(i k_{j}\right)}=-\frac{c_{j}}{2 i k_{j}}, \quad k_{j}>0 ; \quad a_{0}=\frac{f^{\prime}(0,0)}{\dot{f}(0)},
$$

where $\operatorname{Im} a_{j}>0,1 \leq j \leq J$, and $\operatorname{Im} a_{0} \geq 0, a_{0} \neq 0$ iff $f(0)=0$. We prove that if $q \in L_{1,1}$ and $f(0)=0$ then $\dot{f}(0)$ exists and $\dot{f}(0) \neq 0$ (Theorem 3.1.3 below). This is a subtle result.

Lemma 3.1.1. The $I(k)$ equals to the Weyl function $m(k)$.

Proof. The $m(k)$ is a function such that $\theta(x, k)+m(k) \varphi(x, k) \in L^{2}\left(\mathbb{R}_{+}\right)$if $\operatorname{Im} k>0$. Clearly

$$
f(x, k)=c(k)[\theta(x, k)+m(k) \varphi(x, k)],
$$

where $c(k) \neq 0, \operatorname{Im} k>0$. Thus $I(k)=\frac{\theta^{\prime}(0, k)+m(k) \varphi^{\prime}(0, k)}{\theta(0, k)+m(k) \varphi(0, k)}=m(k)$ because of (1.2.3) and (1.2.4).

Our basic uniqueness theorem is:

Theorem 3.1.2. If $q_{j} \in L_{1,1}, j=1,2$, generate the same $I(k)$, then $q_{1}=q_{2}$.

Proof. Let $p:=q_{2}-q_{1}, f_{j}$ be the Jost solution (1.2.5) corresponding to $q_{j}, w:=f_{1}-f_{2}$. Then one has

$$
-w^{\prime \prime}+q_{1} w-k^{2} w=p f_{2}, \quad|w|+\left|w^{\prime}\right|=o(1), \quad x \rightarrow+\infty .
$$

Multiply (3.1.3) by $f_{1}$, integrate over $\mathbb{R}_{+}$, then by parts, using (3.1.3), and get

$$
\begin{aligned}
\int_{0}^{\infty} p f_{2} f_{1} d x=w^{\prime}(0) f_{1}(0)- & w(0) f_{1}^{\prime}(0) \\
& =f_{1}^{\prime}(0, k) f_{2}(k)-f_{2}^{\prime}(0, k) f_{1}(k)=f_{1}(k) f_{2}(k)\left[I_{1}(k)-I_{2}(k)\right]=0 .
\end{aligned}
$$

By property $C_{+}$(Theorem 2.1.1), $p(x)=0$. 
Remark: If $q_{j} \in L_{1,1}, j=1,2$, and $\left(^{*}\right)\left|I_{1}(k)-I_{2}(k)\right| \leq c e^{-2 a \operatorname{Im} k}$, where $k=|k| e^{i \arg k}, \forall|k|>$ $0,0<\arg k<\pi$, then $q_{1}(x)=q_{2}(x)$ for almost all $x \in(0, a)$. This result is proved in [GS1] for $q_{j} \in L_{l o c}^{1}$. Our proof is based on (3.1.4), from which, using $\left(^{*}\right)$, one gets $\left({ }^{*}\right) \int_{0}^{\infty} p f_{2} f_{1} d x=O\left(e^{-2 a \operatorname{Im} k}\right)$. Note that $f_{1} f_{2}=\left(I+V^{*}\right) e^{2 i k x}$, where $V^{*}$ is the adjoint to a Volterra operator (see the formula below (2.1.4)). Thus, $\left(^{* *}\right)$ can be written as $\left({ }^{* * *}\right) \int_{0}^{\infty} p_{1} e^{2 i k x} d x=O\left(e^{-2 a \operatorname{Im} k}\right)$, where $p_{1}:=(I+V) p$. Formulas (6.5.6) (see Chapter 6 below) and (***) imply $p_{1}=0$ for almost all $x \in(0, a)$. Since $V$ is a Volterra operator, it follows that $p=0$ for almost all $x \in(0, a)$, as claimed.

Theorem 3.1.3. If $q \in L_{1,1}$ and $f(0)=0$, then $\dot{f}(0)$ exists and $\dot{f}(0) \neq 0$.

Proof. Let us prove that $f(k)=i k \widetilde{A}_{1}(k), \widetilde{A}_{1}(0) \neq 0, \widetilde{A}_{1}:=\int_{0}^{\infty} e^{i k t} A_{1}(t) d t$, and $A_{1} \in L^{1}\left(\mathbb{R}_{+}\right)$. Let $A_{1}(t):=\int_{t}^{\infty} A(s) d s, \widetilde{A}:=f(k)-1$, and $A(y)=A(0, y)$, where $A(x, y)$ is defined in (1.2.25) and $A(y) \in$ $L^{1}\left(\mathbb{R}_{+}\right)$by (1.2.26). Integrating by parts, one gets $\widetilde{A}(k)=-\left.\exp (i k t) A_{1}(t)\right|_{0} ^{\infty}+i k \widetilde{A}_{1}=i k \widetilde{A}_{1}-1$. Thus $f(k)=i k \widetilde{A}_{1}$. The basic difficulty is to prove that $A_{1} \in L^{1}\left(\mathbb{R}_{+}\right)$. If this is done, then $\lim _{k \rightarrow 0} \frac{f(k)}{k}=\dot{f}(0)$ exists and $\dot{f}(0)=i \widetilde{A}_{1}(0)$. To prove that $\dot{f}(0) \neq 0$, one uses the Wronskian formula (3.2.2) with $x=0$ : $f(-k) f^{\prime}(0, k)-f(k) f^{\prime}(0,-k)=2 i k$. Divide by $k$ and let $k \rightarrow 0$. Since existence of $\dot{f}(0)$ is proved, one gets $-\dot{f}(0) f^{\prime}(0,0)=i$, so $\dot{f}(0) \neq 0$. We have used here the existence of the $\operatorname{limit}_{\lim } \lim _{k \rightarrow 0} f^{\prime}(0, k)=f^{\prime}(0,0)$. The existence of it follows from (1.2.25):

$$
f^{\prime}(0, k)=i k-A(0,0)+\int_{0}^{\infty} A_{x}(0, y) e^{i k y} d y
$$

and

$$
f^{\prime}(0,0)=-A(0,0)+\int_{0}^{\infty} A_{x}(0, y) d y
$$

From (1.2.27) one sees that $A_{x}(0, y) \in L^{1}\left(\mathbb{R}_{+}\right)$. Thus, to complete the proof, one has to prove $A_{1} \in$ $L^{1}\left(\mathbb{R}_{+}\right)$. To prove this, use (1.5.13) with $x=0$ and (1.5.11). Since $f\left(i k_{j}\right)=0$, one has $\widetilde{A}\left(i k_{j}\right)=-1$. Therefore (1.5.13) with $x=0$ yields:

$$
A(y)+\int_{0}^{\infty} A(t) F_{s}(t+y) d t+F_{s}(y)=0, \quad y \geq 0 .
$$

Integrate (3.1.7) over $(x, \infty)$ to get:

$$
A_{1}(x)+\int_{0}^{\infty} A(t) \int_{x}^{\infty} F_{s}(t+y) d y d t+\int_{x}^{\infty} F_{s}(y) d y=0, \quad x \geq 0,
$$

where $F_{s}(y) \in L^{1}\left(\mathbb{R}_{+}\right)$. Integrating by parts yields:

$$
\int_{0}^{\infty} A(t) \int_{x}^{\infty} F_{s}(t+y) d y d t=A_{1}(0) \int_{x}^{\infty} F_{s}(y) d y-\int_{x}^{\infty} A_{1}(t) F_{s}(x+t) d t
$$

Because $0=f(0)=1+\int_{0}^{\infty} A(y) d y$, one has $A_{1}(0)=-1$. Therefore (3.1.8) and (3.1.9) imply:

$$
A_{1}(x)-\int_{x}^{\infty} A_{1}(t) F_{s}(x+t) d t=0, \quad x \geq 0 .
$$

From this equation and from the inclusion $F_{s}(t) \in L^{1}\left(\mathbb{R}_{+}\right)$, one derives $A_{1} \in L^{1}\left(\mathbb{R}_{+}\right)$as follows. Choose a $T(t) \in C_{0}^{\infty}\left(\mathbb{R}_{+}\right)$such that $\left\|F_{s}-T\right\|_{L^{1}\left(\mathbb{R}_{+}\right)} \leq 0.5$, and let $Q:=F_{s}-T$. Then (3.1.10) can be written as:

$$
A_{1}(x)-\int_{x}^{\infty} Q(x+t) A_{1}(t) d t=a(x):=\int_{x}^{\infty} T(x+t) A_{1}(t) d t, \quad x \geq 0 .
$$

Since $T \in C_{0}^{\infty}\left(\mathbb{R}_{+}\right)$and $A \in L^{1}\left(\mathbb{R}_{+}\right)$, it follows that $A_{1}$ is bounded. Thus $a \in L^{1}\left(\mathbb{R}_{+}\right)$. The operator $Q A_{1}:=\int_{x}^{\infty} Q(x+t) A_{1}(t) d t$ has norm $\|Q\|_{L^{1}\left(\mathbb{R}_{+}\right) \rightarrow L^{1}\left(\mathbb{R}_{+}\right)} \leq 0.5$. Therefore equation (3.1.3) is uniquely solvable in $L^{1}\left(\mathbb{R}_{+}\right)$and $A_{1} \in L^{1}\left(\mathbb{R}_{+}\right)$. Theorem 3.1.3 is proved. 


\subsection{Characterization of the $I$-functions}

One has

$$
\operatorname{Im} I(k)=\frac{1}{2 i}\left(\frac{f^{\prime}(0, k)}{f(k)}-\frac{\overline{f^{\prime}(0, k)}}{\overline{f(k)}}\right)=\frac{k}{|f(k)|^{2}},
$$

where the Wronskian formula was used with $x=0$ :

$$
\overline{f(x, k)} f^{\prime}(x, k)-f(x, k) \overline{f^{\prime}(x, k)}=2 i k .
$$

From (1.2.21) and (3.2.1) with $k=\sqrt{\lambda}$, one gets:

$$
\frac{1}{\pi} \operatorname{Im} I(\sqrt{\lambda}) d \lambda=d \rho, \quad \lambda \geq 0
$$

The $I(k)$ determines uniquely the points $i k_{j}, 1 \leq j \leq J$, as the (simple) poles of $I(k)$ on the imaginary axis, and the numbers $c_{j}$ by (3.1.2). Therefore $I(k)$ determines uniquely the spectral function $\rho(\lambda)$ by formula (1.2.21). The characterization of the class of spectral functions $\rho(\lambda)$, given in Section 4.6 , induces a characterization of the class of $I$-functions.

The other characterization of the $I$-functions one obtains by establishing a one-to-one correspondence between the $I$-function and the scattering data $\mathcal{S}$ (1.2.17). Namely, the numbers $k_{j}$ and $J, 1 \leq j \leq J$, are obtained from $I(k)$ since $i k_{j}$ are the only poles of $I(k)$ in $\mathbb{C}_{+}$, the numbers $s_{j}$ are obtained by the formula (see (1.2.16) and (3.1.2)):

$$
s_{j}=-\frac{2 i k_{j}}{a_{j}\left[\dot{f}\left(i k_{j}\right)\right]^{2}},
$$

if $f(k)$ is found from $I(k)$. Finally, $f(k)$ can be uniquely recovered from $I(k)$ by solving a Riemann problem. To derive this problem, define

$$
w(k):=\prod_{j=1}^{J} \frac{k-i k_{j}}{k+i k_{j}} \quad \text { if } \quad I(0)<\infty,
$$

and

$$
w_{0}(k):=\frac{k}{k+i \kappa} w(k), \quad \text { if } I(0)=\infty, \quad \kappa \neq k_{j} \quad \forall j, \quad \kappa>0 .
$$

Assumption (3.2.5), means that $f(0) \neq 0$, and (3.2.6) means $f(0)=0$.

Define

$$
\begin{aligned}
h(k):=w^{-1}(k) f(k), & I(0)<\infty \\
h_{0}(k):=w_{0}^{-1}(k) f(k), & I(0)=\infty .
\end{aligned}
$$

Write $(3.2 .1)$ as $f(k)=\frac{k}{\operatorname{Im} I(k)} \frac{1}{f(-k)}$, or

$$
h_{+}(k)=g(k) h_{-}(k), \quad-\infty<k<\infty,
$$

where $h_{+}(k):=h(k)$ is analytic in $\mathbb{C}_{+}, h_{+}(k) \neq 0$ in $\mathbb{C}_{+}$, the closure of $\mathbb{C}_{+}, h(\infty)=1$ in $\overline{\mathbb{C}_{+}}, h_{-}(k):=$ $h(-k)$ has similar properties in $\mathbb{C}_{-}$,

$$
g(k)=\frac{k}{\operatorname{Im} I(k)} \text { if } I(0)<\infty, \quad g(k)=\frac{k}{\operatorname{Im} I(k)} \frac{k^{2}+1}{k^{2}} \text { if } I(0)=\infty,
$$

$g(k)>0$ for $k>0, g(k)$ is bounded in a neighborhood of $k=0$ and has a finite limit at $k=0$. From (3.2.9) and the properties of $h$, one gets:

$$
h(k)=\exp \left(\frac{1}{2 \pi i} \int_{-\infty}^{\infty} \frac{\ln g(t)}{t-k} d t\right)
$$


and

$$
f(k)=w(k) h(k), \quad \operatorname{Im} k \geq 0 .
$$

In Section 3.4 we prove:

$$
\frac{1}{2 \pi} \int_{-\infty}^{\infty}[I(k)-i k] e^{-i k t} d k=-\frac{r_{0}}{2}-\sum_{j=1}^{J} r_{j} e^{k_{j} t}, \quad t<0,
$$

where $r_{j}=-i a_{j}$. Taking $t \rightarrow-\infty$ in (3.2.13), one finds step by step all the numbers $r_{j}, k_{j}$ and $J$. If $I(0)<\infty$, then $r_{0}=0$. Thus the data (1.2.17) are algorithmically recovered from $I(k)$ known for all $k>0$.

A characterization of $\mathcal{S}$ is given in Section 5.5, and thus an implicit characterization of $I(k)$ is also given.

\subsection{Inversion procedures.}

Both procedures in Section 3.2, which allow one to construct either $\rho(\lambda)$ or $\mathcal{S}$ from $I(k)$ can be considered as inversion procedures $I \Rightarrow q$ because in Chapter 4 and Chapter 5 reconstruction procedures are given for recovery of $q(x)$ from either $\rho(\lambda)$ or $\mathcal{S}$. All three data, $I(k), \rho(\lambda)$ and $\mathcal{S}$ are equivalent. Thus, our inversion schemes are:

$$
\begin{gathered}
I(k) \Rightarrow \rho(\lambda) \Rightarrow q(x), \\
I(k) \Rightarrow \mathcal{S} \Rightarrow q(x),
\end{gathered}
$$

where (1.5.1) gives the details of the step $\rho(\lambda) \Rightarrow q(x)$, and (1.5.10) gives the details of the step $\mathcal{S} \Rightarrow q(x)$.

\subsection{Properties of $I(k)$}

In this section, we derive the following formula for $I(k)$ :

Theorem 3.4.1. One has

$$
I(k)=i k+\sum_{j=0}^{J} \frac{a_{j}}{k-i k_{j}}+\widetilde{a}(k), \quad \widetilde{a}(k)=\int_{0}^{\infty} a(t) e^{i k t} d t,
$$

where $k_{0}$, Im $a_{0}>0$ if and only if $f(0)=0, a_{j}$ are the constants defined in (3.1.2), Im $a_{j}>0,1 \leq j \leq J$, $a(t) \in L^{1}\left(\mathbb{R}_{+}\right)$if $f(0) \neq 0$ and $q \in L_{1,1}, a(t) \in L^{1}\left(\mathbb{R}_{+}\right)$if $f(0)=0$ and $q \in L_{1,3}\left(\mathbb{R}_{+}\right)$.

We prove this result in several steps which are formulated as lemmas. Using (1.2.25) one gets

$$
\begin{aligned}
I(k)= & \frac{i k-A(0,0)+\int_{0}^{\infty} A_{x}(0, y) e^{i k y} d y}{1+\widetilde{A}(k)}, \\
& A(y):=A(0, y), \widetilde{A}(k):=\int_{0}^{\infty} A(y) e^{i k y} d y .
\end{aligned}
$$

One has (cf. (3.2.5))

$$
\begin{gathered}
f(k)=1+\widetilde{A}(k):=f_{0}(k) w(k) \frac{k}{k+i \kappa}, w(k):=\prod_{j=1}^{J} \frac{k-i k_{j}}{k+i k_{j}}, \kappa \neq k_{j} \quad \forall j \\
f_{0}(k) \neq 0 \text { in } \mathbb{C}_{+}, \quad f_{0}(\infty)=1,
\end{gathered}
$$

$f_{0}(k)$ is analytic in $\mathbb{C}_{+}$, the factor $\frac{k}{k+i}$ in (3.4.3) is present if and only if $f(0)=0$, and $w(k) \frac{k}{k+i \kappa}:=w_{0}(k)$. 
Lemma 3.4.2. If $f(0) \neq 0$ and $q \in L_{1,1}\left(\mathbb{R}_{+}\right)$then

$$
f_{0}(k)=1+\tilde{b}_{0}(k), \quad b_{0}(x) \in W^{1,1}\left(\mathbb{R}_{+}\right), \quad\left\|b_{0}\right\|_{W^{1,1}\left(\mathbb{R}_{+}\right)}:=\int_{0}^{\infty}\left(\left|b_{0}\right|+\left|b_{0}^{\prime}\right|\right) d x<\infty .
$$

Proof. It is sufficient to prove that, for any $j, 1 \leq j \leq J$, the function

$$
\frac{k+i k_{j}}{k-i k_{j}} f(k)=1+\int_{0}^{\infty} g_{j}(t) e^{i k t} d t, \quad g_{j} \in W^{1,1}\left(\mathbb{R}_{+}\right) .
$$

Since $\frac{k+i k_{j}}{k-i k_{j}}=1+\frac{2 i k_{j}}{k-i k_{j}}$, and since $A(y) \in W^{1,1}\left(\mathbb{R}_{+}\right)$provided that $q \in L_{1,1}\left(\mathbb{R}_{+}\right)$(see (1.2.26)-(1.2.27)), it is sufficient to check that

$$
\frac{f(k)}{k-i k_{j}}=\int_{0}^{\infty} g(t) e^{i k t} d t, \quad g \in W^{1,1}\left(\mathbb{R}_{+}\right)
$$

Note that

$$
\frac{k-i k_{j}}{k+i k_{j}}=\int_{-\infty}^{\infty} e^{i k t}\left[\delta(t)-2 k_{j} e^{-k_{j} t} \theta(t)\right] d t, \quad \theta(t):= \begin{cases}1, & t \geq 0 \\ 0, & t<0 .\end{cases}
$$

One has $f\left(i k_{j}\right)=0$, thus

$$
\begin{aligned}
\frac{f(k)}{k-i k_{j}} & =\frac{f(k)-f(i k)}{k-i k_{j}}=\int_{0}^{\infty} d y A(y) \frac{e^{i\left(k-i k_{j}\right) y}-1}{k-i k_{j}} e^{-k_{j} y} d y \\
& =\int_{0}^{\infty} d y A(y) e^{-k_{j} y} i \int_{0}^{y} e^{i\left(k-i k_{j}\right) s} d s=\int_{0}^{\infty} e^{i k s} h_{j}(s) d s,
\end{aligned}
$$

where

$$
h_{j}(s):=i \int_{s}^{\infty} A(y) e^{-k_{j}(y-s)} d y=i \int_{0}^{\infty} A(t+s) e^{-k_{j} t} d t .
$$

From (3.4.8) one obtains (3.4.7) since $A(y) \in W^{1,1}\left(\mathbb{R}_{+}\right)$.

Lemma 3.4.2 is proved.

Lemma 3.4.3. If $f(0)=0$ and $q \in L_{1,2}\left(\mathbb{R}_{+}\right)$, then (3.4.5) holds.

Proof. The proof goes as above with one difference: if $f(0)=0$ then $k_{0}=0$ is present in formula (3.4.1) and in formula (3.4.8) with $k_{0}=0$ one has

$$
h_{0}(s)=i \int_{0}^{\infty} A(t+s) d t
$$

Thus, using (1.2.26), one gets

$$
\begin{aligned}
\int_{0}^{\infty}\left|h_{0}(s)\right| d s \leq c \int_{0}^{\infty} d s \int_{0}^{\infty} d t \int_{\frac{t+s}{2}}^{\infty}|q(u)| d u \\
=2 c \int_{0}^{\infty} d s \int_{\frac{s}{2}}^{\infty} d v \int_{v}^{\infty}|q(u)| d u \leq 2 c \int_{0}^{\infty} d s \int_{\frac{s}{2}}^{\infty}|q(u)| u d u \\
=4 c \int_{0}^{\infty} u^{2}|q(u)| d u<\infty \quad \text { if } \quad q \in L_{1,2}\left(\mathbb{R}_{+}\right),
\end{aligned}
$$

where $c>0$ is a constant. Similarly one checks that $h_{0}^{\prime}(s) \in L^{1}\left(\mathbb{R}_{+}\right)$if $q \in L_{1,2}\left(\mathbb{R}_{+}\right)$.

Lemma 3.4.3 is proved.

Lemma 3.4.4. Formula (3.4.1) holds. 
Proof. Write

$$
\frac{1}{f(k)}=\frac{\frac{k+i}{k} \prod_{j=1}^{J} \frac{k+i k_{j}}{k-i k_{j}}}{f_{0}(k)}
$$

Clearly

$$
\frac{k+i}{k} \prod_{j=1}^{J} \frac{k+i k_{j}}{k-i k_{j}}=1+\sum_{j=0}^{J} \frac{c_{j}}{k-i k_{j}}, \quad k_{0}:=0, \quad k_{j}>0 .
$$

By the Wiener-Levy theorem [GRS, §17], one has

$$
\frac{1}{f_{0}(k)}=1+\int_{0}^{\infty} b(t) e^{i k t} d t, \quad b(t) \in W^{1,1}\left(\mathbb{R}_{+}\right),
$$

where $f_{0}(k)$ is defined in (3.4.3). Actually, the Wiener-Levy theorem yields $b(t) \in L^{1}\left(\mathbb{R}_{+}\right)$. However, since $b_{0} \in W^{1,1}\left(\mathbb{R}_{+}\right)$, one can prove that $b(t) \in W^{1,1}\left(\mathbb{R}_{+}\right)$. Indeed, $\widetilde{b}$ and $\widetilde{b}_{0}$ are related by the equation:

$$
\left(1+\widetilde{b}_{0}\right)(1+\widetilde{b})=1, \quad \forall k \in \mathbb{R},
$$

which implies

$$
\widetilde{b}=-\widetilde{b}_{0}-\widetilde{b}_{0} \widetilde{b}
$$

or

$$
b(t)=-b_{0}(t)-\int_{0}^{t} b_{0}(t-s) b(s) d s:=-b_{0}-b_{0} * b,
$$

where $*$ is the convolution operation.

Since $b_{0}^{\prime} \in L^{1}\left(\mathbb{R}_{+}\right)$and $b \in L^{1}\left(\mathbb{R}_{+}\right)$the convolution $b_{0}^{\prime} * b \in L^{1}\left(\mathbb{R}_{+}\right)$. So, differentiating (3.4.10) one sees that $b^{\prime} \in L^{1}\left(\mathbb{R}_{+}\right)$, as claimed.

From the above formulas one gets:

$$
I(k)=\left(i k-A(0)+\widetilde{A}_{1}\right)(1+\widetilde{b})\left(1+\sum_{j=0}^{J} \frac{c_{j}}{k-i k_{j}}\right)=i k+c+\sum_{j=0}^{J} \frac{a_{j}}{k-i k_{j}}+\widetilde{a},
$$

where $c$ is a constant defined in (3.4.13) below, the constants $a_{j}$ are defined in (3.4.14) and the function $\widetilde{a}$ is defined in (3.4.15). We will prove that $c=0$ (see (3.4.17)).

To derive (3.4.11), we have used the formula:

$$
i k \widetilde{b}=i k\left[\left.\frac{e^{i k t}}{i k} b(t)\right|_{0} ^{\infty}-\frac{1}{i k} \int_{0}^{\infty} e^{i k t} b^{\prime}(t) d t\right]=-b(0)-\widetilde{b}^{\prime},
$$

and made the following transformations:

$$
\begin{aligned}
& I(k)=i k-A(0)-b(0)-\widetilde{b}^{\prime}+\widetilde{A}_{1}-A(0) \widetilde{b}+\widetilde{A}_{1} \widetilde{b} \sum_{j=0}^{J} \frac{c_{j} i k}{k-i k_{j}} \\
& -\sum_{j=0}^{J} \frac{c_{j}[A(0)+b(0)]}{k-i k_{j}}+\sum_{j=0}^{J} \frac{\widetilde{g}(k)-\widetilde{g}\left(i k_{j}\right)}{k-i k_{j}} c_{j}+\sum_{j=0}^{J} \frac{\widetilde{g}\left(i k_{j}\right) c_{j}}{k-i k_{j}}
\end{aligned}
$$

where

$$
\widetilde{g}(k):=-\widetilde{b}^{\prime}+\widetilde{A}_{1}-A(0) \widetilde{b}+\widetilde{A}_{1} \widetilde{b} .
$$

Comparing (3.4.12) and (3.4.11) one concludes that

$$
c:=-A(0)-b(0)+i \sum_{j=0}^{J} c_{j}
$$




$$
\begin{gathered}
a_{j}:=-c_{j}\left[k_{j}+A(0)+b(0)-\widetilde{g}\left(i k_{j}\right)\right], \\
\widetilde{a}(k):=\widetilde{g}(k)+\sum_{j=0}^{J} \frac{\widetilde{g}(k)-\widetilde{g}\left(i k_{j}\right)}{k-i k_{j}} c_{j} .
\end{gathered}
$$

To complete the proof of Lemma 3.4.4 one has to prove that $c=0$, where $c$ is defined in (3.4.13). This follows from the asymptotics of $I(k)$ as $k \rightarrow \infty$. Namely, one has:

$$
\widetilde{A}(k)=-\frac{A(0)}{i k}-\frac{1}{i k} \widetilde{A}^{\prime} .
$$

From (3.4.16) and (3.4.2) one gets:

$$
\begin{aligned}
I(k) & =\left(i k-A(0)+\widetilde{A}_{1}\right)\left[1-\frac{A(0)}{i k}+o\left(\frac{1}{k}\right)\right]^{-1} \\
& =\left(i k-A(0)+\widetilde{A}_{1}\right)\left(1+\frac{A(0)}{i k}+o\left(\frac{1}{k}\right)\right)=i k+o(1), \quad k \rightarrow+\infty .
\end{aligned}
$$

From (3.4.17) and (3.4.11) it follows that $c=0$. Lemma 3.4.4 is proved.

Lemma 3.4.5. One has $a_{j}=i r_{j}, r_{j}>0,1 \leq j \leq J$, and $r_{0}=0$ if $f(0) \neq 0$, and $r_{0}>0$ if $f(0)=0$.

Proof. From (3.1.2) one gets:

$$
a_{j}=-\frac{c_{j}}{2 i k_{j}}=i \frac{c_{j}}{2 k_{j}}:=i r_{j}, \quad r_{j}:=\frac{c_{j}}{2 k_{j}}>0, \quad j>0 .
$$

If $j=0$, then

$$
a_{0}=\operatorname{Res}_{k=0} I(k):=\frac{f^{\prime}(0,0)}{\dot{f}(0)} .
$$

Here by $\operatorname{Res}_{k=0} I(k)$ we mean the right-hand side of (3.4.19) since $I(k)$ is, in general, not analytic in a disc centered at $k=0$, it is analytic in $\mathbb{C}_{+}$and, in general, cannot be continued analytically into $\mathbb{C}_{-}$. By Theorem 3.1.3 the right-hand side of (3.4.19) is well defined and

$$
a_{0}=-\frac{i}{[\dot{f}(0)]^{2}}=i r_{0}, \quad r_{0}:=-\frac{1}{[\dot{f}(0)]^{2}} .
$$

From (1.2.25) one gets:

$$
\dot{f}(0)=i \int_{0}^{\infty} A(y) y d y .
$$

Since $A(y)$ is a real-valued function if $q(x)$ is real-valued (this follows from the integral equation (1.2.28), formula (3.4.21) shows that

$$
[\dot{f}(0)]^{2}<0,
$$

and (3.4.20) implies

$$
r_{0}>0 .
$$

Lemma 3.4.5 is proved.

One may be interested in the properties of function $a(t)$ in (3.4.1). These can be obtained from (3.4.15) and (3.4.5) as in the proof of Lemma 3.4.2 and Lemma 3.4.3.

In particular, the statements of Theorem 3.4.1 are obtained. 
Remark 3.4.6. Even if $q(x) \not \equiv 0$ is compactly supported, one cannot claim that $a(t)$ is compactly supported.

Proof. Assume for simplicity that $J=0$ and $f(0) \neq 0$. In this case, if $a(t)$ is compactly supported then $I(k)$ is an entire function of exponential type. It is proved in [R, p.278] that if $q(x) \not \equiv 0$ is compactly supported, $q \in L^{1}\left(\mathbb{R}_{+}\right)$, then $f(k)$ has infinitely many zeros in $\mathbb{C}$. The function $f^{\prime}(0, z) \neq 0$ if $f(z)=0$. Indeed, if $f(z)=0$ and $f^{\prime}(0, z)=0$ then $f(x, z) \equiv 0$ by the uniqueness of the solution of the Cauchy problem for equation (1.2.5) with $k=z$. Since $f(x, z) \not \equiv 0$, one has a contradiction, which proves that $f^{\prime}(0, z) \neq 0$ if $f(z)=0$. Thus $I(k)$ cannot be an entire function if $q(x) \not \equiv 0, q(x) \in L^{1}\left(\mathbb{R}_{+}\right)$and $q(x)$ is compactly supported.

Let us consider the following question:

What are the potentials for which $a(t)=0$ in (3.4.1)?

In other words, let us assume

$$
I(k)=i k+\sum_{j=0}^{J} \frac{i r_{j}}{k-i k_{j}},
$$

and find $q(x)$ corresponding to $I$-function (3.4.24), and describe the decay properties of $q(x)$ as $x \rightarrow+\infty$.

We give two approaches to this problem. The first one is as follows.

By definition

$$
f^{\prime}(0, k)=I(k) f(k), \quad f^{\prime}(0,-k)=I(-k) f(-k), \quad k \in \mathbb{R} .
$$

Using (3.4.25) and (1.2.11) one gets $[I(k)-I(-k)] f(k) f(-k)=2 i k$, or

$$
f(k) f(-k)=\frac{k}{\operatorname{ImI}(k)}, \quad \forall k \in \mathbb{R} .
$$

By (3.4.18) one can write (see (1.2.21)) the spectral function corresponding to the $I$-function (3.4.24) $(\sqrt{\lambda}=k)$ :

$$
d \rho(\lambda)= \begin{cases}\frac{\operatorname{Im} I(\lambda)}{\pi} d \lambda, & \lambda \geq 0 \\ \sum_{j=1}^{J} 2 k_{j} r_{j} \delta\left(\lambda+k_{j}^{2}\right) d \lambda, & \lambda<0\end{cases}
$$

where $\delta(\lambda)$ is the delta-function.

Knowing $d \rho(\lambda)$ one can recover $q(x)$ algorithmically by the scheme (1.5.1).

Consider an example. Suppose $f(0) \neq 0, \quad J=1$,

$$
I(k)=i k+\frac{i r_{1}}{k-i k_{1}}=i k+\frac{i r_{1}\left(k+i k_{1}\right)}{k^{2}+k_{1}^{2}}=i\left(k+\frac{r_{1} k}{k^{2}+k_{1}^{2}}\right)-\frac{r_{1} k_{1}}{k^{2}+k_{1}^{2}} .
$$

Then (3.4.27) yields:

$$
d \rho(\lambda)= \begin{cases}\frac{d \lambda}{\pi}\left(\sqrt{\lambda}+\frac{r_{1} \sqrt{\lambda}}{\lambda+k_{1}^{2}}\right), & \lambda>0, \\ 2 k_{1} r_{1} \delta\left(\lambda+k_{1}^{2}\right) d \lambda, & \lambda<0 .\end{cases}
$$

Thus (1.5.3) yields:

$$
L(x, y)=\frac{1}{\pi} \int_{0}^{\infty} d \lambda \frac{r_{1} \sqrt{\lambda}}{\lambda+k_{1}^{2}} \frac{\sin \sqrt{\lambda} x}{\sqrt{\lambda}} \frac{\sin \sqrt{\lambda} y}{\sqrt{\lambda}}+2 k_{1} r_{1} \frac{\operatorname{sh}\left(k_{1} x\right)}{k_{1}} \frac{\operatorname{sh}\left(k_{1} y\right)}{k_{1}}
$$


and, setting $\lambda=k^{2}$ and taking for simplicity $2 k_{1} r_{1}=1$, one finds:

$$
\begin{aligned}
L_{0}(x, y) & :=\frac{2 r_{1}}{\pi} \int_{0}^{\infty} \frac{d k k^{2}}{k^{2}+k_{1}^{2}} \frac{\sin (k x) \sin (k y)}{k^{2}} \\
& =\frac{2 r_{1}}{\pi} \int_{0}^{\infty} \frac{d k \sin (k x) \sin (k y)}{k^{2}+k_{1}^{2}} \\
& =\frac{r_{1}}{\pi} \int_{0}^{\infty} \frac{d k[\cos k(x-y)-\cos k(x+y)]}{k^{2}+k_{1}^{2}} \\
& =\frac{r_{1}}{2 k_{1}}\left(e^{-k_{1}|x-y|}-e^{-k_{1}(x+y)}\right), \quad k_{1}>0,
\end{aligned}
$$

where the known formula was used:

$$
\frac{1}{\pi} \int_{0}^{\infty} \frac{\cos k x}{k^{2}+a^{2}} d k=\frac{1}{2 a} e^{-a|x|}, \quad a>0, \quad x \in \mathbb{R} .
$$

Thus

$$
L(x, y)=\frac{r_{1}}{2 k_{1}}\left[e^{-k_{1}|x-y|}-e^{-k_{1}(x+y)}\right]+\frac{s h\left(k_{1} x\right)}{k_{1}} \frac{s h\left(k_{1} y\right)}{k_{1}} .
$$

Equation (1.5.4) with kernel (3.4.33) is not an integral equation with degenerate kernel:

$$
\begin{aligned}
K(x, y) & +\int_{0}^{x} K(x, t)\left[\frac{e^{-k_{1}|t-y|}-e^{-k_{1}(t+y)}}{2 k_{1} / r_{1}}+\frac{s h\left(k_{1} t\right)}{k_{1}} \frac{s h\left(k_{1} y\right)}{k_{1}}\right] d t \\
& =-\frac{e^{-k_{1}|x-y|}-e^{-k_{1}(x+y)}}{2 k_{1} / r_{1}}-\frac{\operatorname{sh}\left(k_{1} x\right)}{k_{1}} \frac{\operatorname{sh}\left(k_{1} y\right)}{k_{1}} .
\end{aligned}
$$

This equation can be solved analytically [Ra], but the solution is long. By this reason we do not give the theory developed in [Ra], but give the second approach to a study of the properties of $q(x)$ given $I(k)$ of the form (3.4.28). This approach is based on the theory of the Riemann problem [G].

Equations (3.4.26) and (3.4.28) imply

$$
f(k) f(-k)=\frac{k^{2}+k_{1}^{2}}{k^{2}+\nu_{1}^{2}}, \quad \nu_{1}^{2}:=k_{1}^{2}+r_{1} .
$$

The function

$$
f_{0}(k):=f(k) \frac{k+i k_{1}}{k-i k_{1}} \neq 0 \quad \text { in } \quad \mathbb{C}_{+} .
$$

Write (3.4.35) as

$$
f_{0}(k) \frac{k-i k_{1}}{k+i k_{1}} f_{0}(-k) \frac{k+i k_{1}}{k-i k_{1}}=\frac{k^{2}+k_{1}^{2}}{k^{2}+\nu_{1}^{2}} .
$$

Thus

$$
f_{0}(k)=\frac{k^{2}+k_{1}^{2}}{k^{2}+\nu_{1}^{2}} h, \quad h(k):=\frac{1}{f_{0}(-k)} .
$$

The function $f_{0}(-k) \neq 0$ in $\mathbb{C}_{-}, f_{0}(\infty)=1$ in $\mathbb{C}_{-}$, so $h:=\frac{1}{f_{0}(-k)}$ is analytic in $\mathbb{C}_{-}$.

Consider (3.4.37) as a Riemann problem. One has

$$
\operatorname{ind}_{\mathbb{R}} \frac{k^{2}+k_{1}^{2}}{k^{2}+\nu_{1}^{2}}:=\frac{1}{2 \pi i} \int_{-\infty}^{\infty} d \ln \frac{k^{2}+k_{1}^{2}}{k^{2}+\nu_{1}^{2}}=0
$$

Therefore (see [G]) problem (3.4.37) is uniquely solvable. Its solution is:

$$
f_{0}(k)=\frac{k+i k_{1}}{k+i \nu_{1}}, \quad h(k)=\frac{k-i \nu_{1}}{k-i k_{1}},
$$


as one can check.

Thus, by (3.4.36),

$$
f(k)=\frac{k-i k_{1}}{k+i \nu_{1}} .
$$

The corresponding $S$-matrix is:

$$
S(k)=\frac{f(-k)}{f(k)}=\frac{\left(k+i k_{1}\right)\left(k+i \nu_{1}\right)}{\left(k-i k_{1}\right)\left(k-i \nu_{1}\right)}
$$

Thus

$$
\begin{gathered}
F_{s}(x):=\frac{1}{2 \pi} \int_{-\infty}^{\infty}[1-S(k)] e^{i k x} d k=O\left(e^{-k_{1} x}\right) \text { for } \quad x>0, \\
F_{d}(x)=s_{1} e^{-k_{1} x}
\end{gathered}
$$

and

$$
F(x)=F_{s}(x)+F_{d}(x)=O\left(e^{-k_{1} x}\right) .
$$

Equation (1.5.13) implies $A(x, x)=O\left(e^{-2 k_{1} x}\right)$, so

$$
q(x)=O\left(e^{-2 k_{1} x}\right), \quad x \rightarrow+\infty .
$$

Thus, if $f(0) \neq 0$ and $a(t)=0$ then $q(x)$ decays exponentially at the rate determined by the number $k_{1}, k_{1}=\min _{1 \leq j \leq J} k_{j}$.

If $f(0)=0, J=0$, and $a(t)=0$, then

$$
\begin{gathered}
I(k)=i k+\frac{i r_{0}}{k}, \\
f(k) f(-k)=\frac{k^{2}}{k^{2}+r_{0}}, \quad r_{0}>0 .
\end{gathered}
$$

Let $f_{0}(k)=\frac{(k+i) f(k)}{k}$. Then equation (3.4.46) implies:

$$
f_{0}(k) f_{0}(-k)=\frac{k^{2}+1}{k^{2}+\nu_{0}^{2}}, \quad \nu_{0}^{2}:=r_{0},
$$

and $f_{0}(k) \neq 0$ in $\mathbb{C}_{+}$.

Thus, since ind $\frac{k^{2}+1}{k^{2}+\nu_{0}^{2}}=0, f_{0}(k)$ is uniquely determined by the Riemann problem (3.4.47).

One has:

$$
f_{0}(k)=\frac{k+i}{k+i \nu_{0}}, \quad f_{0}(-k)=\frac{k-i}{k-i \nu_{0}},
$$

and

$$
\begin{aligned}
f(k) & =\frac{k}{k+i \nu_{0}}, \quad S(k)=\frac{f(-k)}{f(k)}=\frac{k+i \nu_{0}}{k-i \nu_{0}}, \\
F_{s}(x) & =\frac{1}{2 \pi} \int_{-\infty}^{\infty}\left(1-\frac{k+i \nu_{0}}{k-i \nu_{0}}\right) e^{i k x} d k \\
& =\frac{-2 i \nu_{0}}{2 \pi} \int_{-\infty}^{\infty} \frac{e^{i k x} d k}{k-i \nu_{0}}=2 \nu_{0} e^{-\nu_{0} x}, \quad x>0,
\end{aligned}
$$

and $F_{d}(x)=0$.

So one gets:

$$
F(x)=F_{s}(x)=2 \nu_{0} e^{-\nu_{0} x}, \quad x>0 .
$$


Equation (1.5.13) yields:

$$
A(x, y)+2 \nu_{0} \int_{x}^{\infty} A(x, t) e^{-\nu_{0}(t+y)} d t=-2 \nu_{0} e^{-\nu_{0}(x+y)}, \quad y \geq x \geq 0 .
$$

Solving (3.4.50) yields:

$$
A(x, y)=-2 \nu_{0} e^{-\nu_{0}(x+y)} \frac{1}{1+e^{-2 \nu_{0} x}} .
$$

The corresponding potential (1.5.12) is

$$
q(x)=O\left(e^{-2 \nu_{0} x}\right), \quad x \rightarrow \infty .
$$

If $q(x)=O\left(e^{-k x}\right), k>0$, then $a(t)$ in (3.4.1) decays exponentially. Indeed, in this case $b^{\prime}(t), A_{1}(y), b(t)$, $A_{1} * b$ decay exponentially, so $g(t)$ decays exponentially, and, by (3.4.15), the function $\frac{\widetilde{g}(k)-\widetilde{g}\left(i k_{j}\right)}{k-i k_{j}}:=\widetilde{h}$ with $h(t)$ decaying exponentially. We leave the details to the reader. 


\section{Chapter 4}

\section{Inverse spectral problem}

\subsection{Auxiliary results}

\subsubsection{Transformation operators}

If $A_{1}$ and $A_{2}$ are linear operators in a Banach space $X$, and $T$ is a boundedly invertible linear operator such that $A_{1} T=T A_{2}$, then $T$ is called a transformation (transmutation) operator. If $A_{2} f=\lambda f$ then $A_{1} T f=\lambda T f$, so that $T$ sends eigenfunctions of $A_{2}$ into eigenfunctions of $A_{1}$ with the same eigenvalue. Let $\ell_{j}=-\frac{d^{2}}{d x^{2}}+q_{j}(x), j=1,2$, be selfadjoint in $H:=L^{2}(0, \infty)$ operators generated by the Dirichlet boundary condition at $x=0$. Other selfadjoint boundary conditions can be considered also, for example, $u^{\prime}(0)-h u(0)=0, h=$ const $\geq 0$.

Theorem 4.1.1. Transformation operator for a pair $\left\{\ell_{1}, \ell_{2}\right\}$ exists and is of the form $T f=(I+K) f$, where the operator $I+K$ is defined in (1.2.24) and the kernel $K(x, y)$ is the unique solution to the problem:

$$
\begin{gathered}
K_{x x}(x, y)-q_{1}(x) K(x, y)=K_{y y}-q_{2}(y) K \\
K(x, 0)=0 \\
K(x, x)=\frac{1}{2} \int_{0}^{x}\left(q_{1}-q_{2}\right) d y .
\end{gathered}
$$

Proof. Consider for simplicity the case $q_{2}=0, q_{1}=q$. The proof is similar in the case $q_{2} \neq 0$. If $q_{2}=0$, then (4.1.3) can be written as

$$
q(x)=2 \frac{d K(x, x)}{d x}, \quad K(0,0)=0 .
$$

If $\ell_{1} T f=T \ell_{2} f$ and $T f=f+\int_{0}^{x} K(x, y) f d y$, then

$$
\begin{aligned}
-f^{\prime \prime}+ & q(x) f+q T f-[K(x, x) f]^{\prime}-\frac{\partial K(x, x)}{\partial x} f-\int_{0}^{x} K_{x x} f d y \\
& =-f^{\prime \prime}-\int_{0}^{x} K(x, y) f_{y y} d y=-\int_{0}^{x} K_{y y} f d y-\left.K(x, y) f^{\prime}\right|_{0} ^{x}+\left.K_{y} f\right|_{0 .} ^{x}
\end{aligned}
$$

Since $f \in D\left(\ell_{1}\right), f(0)=0$, and $f$ is arbitrary otherwise, (4.1.5) implies (4.1.1),(4.1.2) and (4.1.4). Conversely, if $K(x, y)$ solves $(4.1 .1),(4.1 .2)$ and $(4.1 .4)$, then $I+K$ is the transformation operator. To 
finish the proof of Theorem 4.1.1 we need to prove existence of the solution to (4.1.1), (4.1.2) and (4.1.4). Let $\xi=x+y, \eta=x-y, K(x, y):=B(\xi, \eta)$. Then (4.1.1), (4.1.2) and (4.1.4) can be written as

$$
B_{\xi \eta}=\frac{1}{4} q\left(\frac{\xi+\eta}{2}\right) B, B(\xi, 0)=\frac{1}{2} \int_{0}^{\xi / 2} q(s) d s, B(\xi, \xi)=0 .
$$

Integrate (4.1.6) to get

$$
B_{\xi}(\xi, \eta)=\frac{1}{4} q\left(\frac{\xi}{2}\right)+\frac{1}{4} \int_{0}^{\eta} q\left(\frac{\xi+\eta}{x}\right) B(\xi, \tau) d \tau
$$

Integrate (4.1.7) with respect to $\xi$ over $(\eta, \xi)$ and get

$$
B(\xi, \eta)=\frac{1}{4} \int_{\eta}^{\xi} q\left(\frac{s}{2}\right) d s+\frac{1}{4} \int_{\eta}^{\xi} \int_{0}^{\eta} q\left(\frac{s+\tau}{2}\right) B(s, \tau) d \tau d s .
$$

This is a Volterra integral equation which has a solution, this solution is unique, and it can be obtained by iterations.

Theorem 4.1.1 is proved.

\subsubsection{Spectral function}

Consider the problem (1.2.1). The classical result, going back to Weyl, is:

Theorem 4.1.2. There exists a monotone increasing function $\rho(\lambda)$, possibly nonunique, such that for every $h \in L^{2}(0, \infty)$, there exists $\widetilde{h}(\lambda) \in L^{2}(\mathbb{R} ; d \rho)$ such that

$$
\int_{0}^{\infty}|h|^{2} d x=\int_{-\infty}^{\infty}|\widetilde{h}|^{2} d \rho(\lambda), \quad \widetilde{h}(\lambda):=\lim _{n \rightarrow \infty} \int_{0}^{n} f(x) \varphi(x, \sqrt{\lambda}) d x,
$$

where the limit is understood in $L^{2}(\mathbb{R}, d \rho)$ sense. If the potential $q$ in (1.2.1) generates the Dirichlet operator $\ell$ in the limit point at infinity case, then $\rho(\lambda)$ is uniquely defined by $q$, otherwise $\rho(\lambda)$ is defined by $q$ nonuniquely.

The spectral function of $\ell$ has the following properties:

$$
\int_{-\infty}^{0} e^{x|\lambda|^{1 / 2}} d \rho(\lambda)<\infty, \forall x>0, \quad \rho(\lambda)=c \lambda^{3 / 2}+o\left(\lambda^{3 / 2}\right), \quad c=\text { const } \quad \lambda \rightarrow+\infty .
$$

For the boundary condition $u^{\prime}(0)=h u(0), h=$ const $<\infty$, the asymptotics is $\rho(\lambda)=\frac{2}{\pi} \lambda^{1 / 2}+$ $o\left(\lambda^{1 / 2}\right), \quad \lambda \rightarrow+\infty$.

Theorem 4.1.3. (Weyl). For any $\lambda, \operatorname{Im} \lambda \neq 0$, there exists $m(\lambda)$ such that

$$
W(x, \lambda):=\varphi(x, \lambda)+m(\lambda) \varphi(x, \lambda) \in L^{2}\left(\mathbb{R}_{+}\right) .
$$

The function $m(\lambda)$ is analytic in $\mathbb{C}_{+}$and in $\mathbb{C}_{-}$.

The function $m(\lambda)$ is called Weyl's function, or $m$-function, and $W$ is Weyl's solution. Theorem 4.1.2 and Theorem 4.1.3 are proved in $[\mathrm{M}]$. 


\subsection{Uniqueness theorem}

Let $\rho(\lambda)$ be a non-decreasing function of bounded variation on every compact subset of the real axis. Let $h \in L_{0}^{2}\left(\mathbb{R}_{+}\right)$, where $L_{0}^{2}\left(\mathbb{R}_{+}\right)$is a subset of $L^{2}\left(\mathbb{R}_{+}\right)$functions which vanish near infinity. Let $\varphi_{0}:=\frac{\sin (x \sqrt{\lambda})}{\sqrt{\lambda}}$ and

$$
H(\lambda)=\int_{0}^{\infty} h(x) \varphi_{0}(x, \lambda) d x
$$

Our first assumption $\left.A_{1}\right)$ on $\rho(\lambda)$ is:

$$
\int_{-\infty}^{\infty} H^{2}(\lambda) d \rho(\lambda)=0, \quad \Rightarrow h(x)=0 .
$$

This implication should hold for any $h \in L_{0}^{2}\left(\mathbb{R}_{+}\right)$. It holds, for example, if $d \rho(\lambda) \neq 0$ on a set which has a finite limit point: in this case the entire function of $\lambda, H(\lambda)$, vanishes identically, and thus $h=0$.

Denote by $\mathcal{P}$ a subset of $\rho(\lambda)$ and assume that if $\rho_{1}, \rho_{2} \in \mathcal{P}, \nu:=\rho_{1}-\rho_{2}$, and $\mathcal{H}:=\{H(\lambda): h \in$ $\left.C_{0}^{\infty}\left(\mathbb{R}_{+}\right)\right\}$, where $H(\lambda)$ is defined in (4.2.1), then

$$
\left\{\int_{-\infty}^{\infty} H^{2}(\lambda) d \nu(\lambda)=0 \quad \forall H \in \mathcal{H}\right\} \Rightarrow \nu(\lambda)=0
$$

Our second assumption $\left.A_{2}\right)$ on $\rho(\lambda)$ is:

$$
\rho \in \mathcal{P} \text {. }
$$

Let us start with two lemmas.

Lemma 4.2.1. Spectral functions $\rho(\lambda)$ of an operator $\ell_{q}=-\frac{d^{2}}{d x^{2}}+q(x)$ in the limit-point at infinity case belong to $\mathcal{P}$.

Proof. Let $\rho_{1}, \rho_{2}$ be two spectral functions corresponding to $\ell_{1}$ and $\ell_{2}, \ell_{j}=\ell_{q_{j}}, j=1,2, \nu=\rho_{1}-\rho_{2}$ and (*) $\int_{-\infty}^{\infty} H^{2}(\lambda) d \nu=0 \forall h \in L_{0}^{2}\left(\mathbb{R}_{+}\right)$. Let $I+V$ and $I+W$ be the transformation operators corresponding to $\ell_{1}$ and $\ell_{2}$ respectively, such that

$$
\varphi_{0}=(I+V) \varphi_{1}=(I+W) \varphi_{2},
$$

where $\varphi_{j}$ is the regular solution (1.2.1) corresponding to $q_{j}$. Condition $(*)$ implies

$$
\left\|\left(I+V^{*}\right) h\right\|=\left\|\left(I+W^{*}\right) h\right\| \quad \forall h \in L^{2}(0, b),
$$

where, for example,

$$
V h=\int_{a}^{x} V(x, y) h(y) d y, \quad V^{*} h=\int_{x}^{b} V(y, x) h(y) d y .
$$

It follows from (4.2.6) that

$$
I+V^{*}=U\left(I+W^{*}\right),
$$

where $U$ is a unitary operator in $L^{2}(0, b)$. Indeed, $U$ is an isometry and it is surjective because $I+V^{*}$ is.

To finish the proof, one uses Lemma 4.2.2 below and concludes from (4.2.8) that $V^{*}=W^{*}$, so $V=W, \varphi_{1}=\varphi_{2}$, and $q_{1}=q_{2}:=q$. Since, by assumption, $q$ is in the limit-point at infinity case, there is only one spectral function $\rho$ corresponding to $q$, so $\rho_{1}=\rho_{2}=\rho$.

Lemma 4.2.2. If $U$ is unitary and $V$ and $W$ are Volterra operators, then (4.2.8) implies $V=W$. 
Proof. From (4.2.8) one gets $I+V=(I+W) U^{*}$. Since $U$ is unitary, one has $(I+V)\left(I+V^{*}\right)=$ $(I+W)\left(I+W^{*}\right)$. Because $V$ is a Volterra operator, $(I+V)^{-1}=I+V_{1}$, where $V_{1}$ is also a Volterra (of the same type as $V$ in (4.2.7)). Thus, $\left(I+V_{1}\right)(I+W)=\left(I+V^{*}\right)\left(I+W_{1}^{*}\right)$, or

$$
V_{1}+W+V_{1} W=V^{*}+W_{1}^{*}+V^{*} W_{1}^{*}
$$

The left-hand side in (4.2.9) is a Volterra operator of the type $V$ in (4.2.7), while its right-hand side is a Volterra operator of the type $V^{*}$. Since they are equal, each of them must be equal to zero. Thus, $V_{1}(I+W)=-W$, or $(I+V)^{-1}(I+W)=I$, or $V=W$.

Theorem 4.2.3. (Marchenko) The spectral function determines $\ell_{q}$ uniquely.

Proof. If $\ell_{q_{1}}$ and $\ell_{q_{2}}$ have the same spectral function $\rho(\lambda)$, then

$$
\|h\|^{2}=\int_{-\infty}^{\infty}\left|H_{1}(\lambda)\right|^{2} d \rho=\int_{-\infty}^{\infty}\left|H_{2}(\lambda)\right|^{2} d \rho \quad \forall h \in L_{0}^{2}(0, b),
$$

where

$$
H_{j}(\lambda):=\int_{0}^{b} h(x) \varphi_{j}(x, k) d x, \quad k=\sqrt{\lambda}, \quad j=1,2 .
$$

Let $I+K$ be the transformation operator $\varphi_{2}=(I+K) \varphi_{1}$, and $g:=\left(I+K^{*}\right) h$. Then $H_{2}=\left(h, \varphi_{2}\right)=$ $\left(h,(I+K) \varphi_{1}\right)=\left(g, \varphi_{1}\right)$. From (4.2.10) one gets $\|h\|=\left\|\left(I+K^{*}\right) h\right\|$. Thus $I+K^{*}$ is isometric, and, because $K^{*}$ is a Volterra operator, the range of $I+K^{*}$ is the whole space $L^{2}(0, b)$. Therefore $I+K^{*}$ is unitary. This implies $K^{*}=0$. Indeed, $\left(I+K^{*}\right)^{-1}=I+K$ (unitarity) and $\left(I+K^{*}\right)^{-1}=I+V^{*}$ (Volterra property of $K^{*}$ ). Thus $K=V^{*}$, so $K=V^{*}=0$. Therefore $\varphi_{2}=\varphi_{1}$ and $q_{1}=q_{2}$, so $\ell_{q_{1}}=\ell_{q_{2}}$.

Remark 4.2.4. If $\rho_{1}=c \rho_{2}, c=$ const $>0$, then the above argument is applicable and shows that $c$ must be equal to $1, c=1$ and $q_{1}=q_{2}$. Indeed, the above argument yields the unitarity of the operator $\sqrt{c}\left(I+K^{*}\right)$, which implies $c=1$ and $K^{*}=0$.

Here the following lemma is useful:

Lemma 4.2.5. If $b I+Q=0$, where $b=$ const and $Q$ is a compact linear operator, then $b=0$ and $Q=0$.

A simple proof is left to the reader.

\subsection{Reconstruction procedure}

Assume that $\rho(\lambda)$, the spectral function corresponding to $\ell_{q}$, is given. How can one reconstruct $\ell_{q}$, that is, to find $q(x)$ ? We assume for simplicity the Dirichlet boundary condition at $x=0$, but the method allows one to reconstruct the boundary condition without knowing it a priori.

The reconstruction procedure (the Gel'fand-Levitan or GL procedure) is given in (1.5.1)-(1.5.4). Its basic step consists of the derivation of equation (1.5.4) and of a study of this equation.

Let us derive (1.5.4) .

We start with the formula

$$
\int_{-\infty}^{\infty} \varphi(x, \sqrt{\lambda}) \varphi(y, \sqrt{\lambda}) d \rho(\lambda)=\delta(x-y)
$$

and assume that $L(x, y)$ is a continuous function of $x, y$ in $[0, b) \times[0, b)$ for any $b \in(0, \infty)$. 
If $0 \leq y<x$, one gets from (4.3.1) the relation:

$$
\int_{-\infty}^{\infty} \varphi(x, \sqrt{\lambda}) \varphi(y, \sqrt{\lambda}) d \rho(\lambda)=0, \quad 0 \leq y<x .
$$

Using (1.2.24), one gets $\varphi_{0}=(I+K)^{-1} \varphi$. Applying $(I+K)^{-1}$ to $\varphi(y, \sqrt{\lambda})$ in $(4.3 .2)$, one gets

$$
0=\int_{-\infty}^{\infty} \varphi(x, \sqrt{\lambda}) \varphi_{0}(y, \sqrt{\lambda}) d \rho:=I(x, y), \quad 0 \leq y<x .
$$

The right-hand side can be rewritten as:

$$
\begin{aligned}
& I(x, y)= \int_{-\infty}^{\infty}\left(\varphi_{0}+K \varphi_{0}(x) \varphi_{0}(y, \sqrt{\lambda}) d\left(\rho-\rho_{0}\right)\right. \\
&+\int_{-\infty}^{\infty}\left(\varphi_{0}+K \varphi_{0}\right)(x) \varphi_{0}(y, \sqrt{\lambda}) d \rho_{0} \\
&= L(x, y)+\int_{0}^{x} K(x, s) L(s, y) d s+\delta(x-y)+\int_{0}^{x} K(x, s) \delta(s-y) d s \\
&=L(x, y)+\int_{0}^{x} K(x, s) L(s, y) d s+K(x, y), \quad 0 \leq y<x .
\end{aligned}
$$

From (4.3.3) and (4.3.4) one gets, using continuity at $y=x$, equation (1.5.4).

In the above proof the integrals (4.3.2)-(4.3.4) are understood in the distributional sense. If the first inequality (4.1.10) holds, then the above integrals over $(-\infty, n)$ are well defined in the classical sense. If one assumes that the integral in (4.3.5) converges to a function $L(x)$ which is twice differentiable in the classical sense:

$$
L(x):=\lim _{n \rightarrow \infty} L_{n}(x):=\lim _{n \rightarrow \infty} \int_{-\infty}^{n} \frac{1-\cos (x \sqrt{\lambda})}{2 \lambda} d \sigma(\lambda),
$$

then the above proof can be understood in the classical sense, provided that $(*) \sup _{n, x \in(a, b)}\left|L_{n}(x)\right| \leq$ $c(a, b)$ for any $-\infty<a<b<\infty$. If $\rho(\lambda)$ is a spectral function corresponding to $\ell$, then the sequence $L_{n}(x)$ satisfies $(*)$. It is known (see $\left.[\mathrm{L}]\right)$ that the sequence

$$
\Phi_{n}(x, y)=\int_{-\infty}^{n} \varphi(x, \sqrt{\lambda}) \varphi(y, \sqrt{\lambda}) d \rho(\lambda)-\int_{-\infty}^{n} \frac{\sin (x \sqrt{\lambda}) \sin (y \sqrt{\lambda})}{\lambda} d \rho_{0}(\lambda)
$$

satisfies $(*)$ and converges to zero.

Lemma 4.3.1. Assume (4.2.2) and suppose that the function $L(x) \in H_{l o c}^{1}\left(\mathbb{R}_{+}\right)$,

$$
L(x):=\int_{-\infty}^{\infty} \frac{1-\cos (x \sqrt{\lambda})}{2 \lambda} d \sigma(\lambda) .
$$

Then equation (1.5.4) has a solution in $L^{2}(0, b)$ for any $b>0$, and this solution is unique.

Proof. Equation (1.5.4) is of Fredholm-type: its kernel

$$
L(x, y)=L(x+y)-L(x-y), \quad L(x, x)=L(2 x), \quad L(0)=0,
$$

is in $H^{1}(0, b) \times H^{1}(0, b)$ for any $b \in(0, \infty)$. Therefore Lemma 4.3.1 is proved if it is proved that the homogeneous version of (1.5.4) has only the trivial solution. Let

$$
h(y)+\int_{0}^{x} L(s, y) h(s) d s=0, \quad 0 \leq y \leq x, \quad h \in L^{2}(0, x) .
$$


Because $L(x, y)$ is a real-valued function, one may assume that $h(y)$ is real-valued. Multiply (4.3.7) by $h(y)$, integrate over $(0, x)$, and use (4.2.1), (1.5.3) and Parseval's equation to get

$$
0=\|h\|^{2}+\int_{-\infty}^{\infty} H^{2}(\lambda) d \sigma=\|h\|^{2}+\int_{-\infty}^{\infty} H^{2}(\lambda) d \rho-\|h\|^{2}=\int_{-\infty}^{\infty} H^{2}(\lambda) d \rho .
$$

From (4.2.2) and (4.3.8) it follows that $h=0$.

If the kernel $K(x, y)$ is found from equation (1.5.4), then $q(x)$ is found by formula (4.1.4).

\subsection{Invertibility of the reconstruction steps}

Our basic result is:

Theorem 4.4.1. Assume (4.2.2), (4.2.3), and suppose $L(x) \in H_{l o c}^{1}\left(\mathbb{R}_{+}\right)$. Then each of the steps in (1.5.1) is invertible, so that (1.5.5) holds.

Proof. 1. Step. $\rho \Rightarrow L$ is done by formula (1.5.3). Let us prove $L \Rightarrow \rho$. If there are $\rho_{1}$ and $\rho_{2}$ corresponding to the same $L(x, y)$, and $\nu:=\rho_{1}-\rho_{2}$, then

$$
0=\int_{-\infty}^{\infty} \varphi_{0}(x, \sqrt{\lambda}) \varphi_{0}(y, \sqrt{\lambda}) d \nu .
$$

Multiply (4.4.1) by $h(x) h(y), h \in C_{0}^{\infty}\left(\mathbb{R}_{+}\right)$, use (4.2.1) and get

$$
0=\int_{-\infty}^{\infty} H^{2}(\lambda) d \nu(\lambda) \quad \forall H \in \mathcal{H}
$$

By (4.2.3) it follows that $\nu=0$, so $\rho_{1}=\rho_{2}$. Thus $L \Rightarrow \rho$.

2. Step. $L \Rightarrow K$ is done by solving (1.5.4). Lemma 4.3 .1 says that $K$ is uniquely determined by $L$. Let us do the step $K \Rightarrow L$. Put $y=x$ in (1.5.4), use (4.3.5) and (4.3.6) and get:

$$
L(2 x)+\int_{0}^{x} K(x, s)[L(x+s)-L(x-s)] d s=-K(x, x),
$$

or

$$
L(2 x)+\int_{x}^{2 x} K(x, y-x) L(y) d y-\int_{0}^{x} K(x, x-y) L(y) d y=-K(x, x) .
$$

This is a Volterra integral equation for $L(x)$ which has a solution and the solution is unique. Thus the step $K \Rightarrow L$ is done. The functions $L(x)$ and $K(x, x)$ are of the same smoothness.

3. Step. $K \Rightarrow q$ is done by formula (4.1.4), $q(x)$ is one derivative less smooth than $K(x, x)$ and therefore one derivative less smooth than $L(x)$. Thus $q \in L_{l o c}^{2}\left(\mathbb{R}_{+}\right)$. The step $q \Rightarrow K$ is done by solving the Goursat problem (4.1.1), (4.1.2), (4.1.4) (with $q_{2}=0$ ), or, equivalently, by solving Volterra equation (4.1.8), which is solvable and has a unique solution. The corresponding $K(x, y)$ is in $H_{\text {loc }}^{1}\left(\mathbb{R}_{+} \times \mathbb{R}_{+}\right)$if $q \in L_{\text {loc }}^{2}\left(\mathbb{R}_{+}\right)$.

Theorem 4.4.1 is proved.

Let us prove that the $q$ obtained by formula (4.1.4) generates the function $K_{1}(x, y)$ identical to the function $K$ obtained in Step 2. The idea of the proof is to show that both $K$ and $K_{1}$ solve the problem (4.1.1), (4.1.2), (4.1.4) with the same $q_{1}=q$ and $q_{2}=0$. This is clear for $K_{1}$. In order to prove it for $K$, it is sufficient to derive from equation (1.5.4) equations (4.1.1) and (4.1.2) with $q$ given by (4.1.4). Let us do this. Equation (4.1.2) follows from (1.2.5) because $L(x, 0)=0$. Define $D:=\frac{\partial^{2}}{\partial x^{2}}-\frac{\partial^{2}}{\partial y^{2}}:=\partial_{x}^{2}-\partial_{y}^{2}$. 
Apply $D$ to (1.5.4) assuming $L(x, y)$ twice differentiable with respect to $x$ and $y$, in which case $K(x, y)$ is also twice differentiable. (See Remark 4.4.3). By (4.3.6), $D L=0$, so

$$
\begin{aligned}
D K & +\frac{d}{d x}[K(x, x) L(x, y)]+K_{x}(x, x) L(x, y) \\
& +\int_{0}^{x} K_{x x}(x, s) L(s, y) d s-\int_{0}^{x} K(x, s) L_{y y}(s, y) d s=0 .
\end{aligned}
$$

Integrate by parts the last integral, (use (4.1.2)), and get

$$
\begin{aligned}
(D K)(x, y) & +\int_{0}^{x}(D K)(x, s) L(s, y) d s \\
& +\dot{K} L+\left(K_{x}+K_{y}\right) L(x, y)+K\left(L_{x}(x, y)-\left.L_{s}(s, y)\right|_{s=x}\right)=0, \quad 0 \leq y \leq x,
\end{aligned}
$$

where $K=K(x, x), L=L(x, y), \dot{K}=\frac{d K(x, x)}{d x}, K_{x}+K_{y}=\dot{K}$, and $L_{x}(x, y)-\left.L_{s}(s, y)\right|_{s=x}=0$. Subtract from (4.4.5) equation (1.5.4) multiplied by $q(x)$, denote $D K(x, y)-q(x) K(x, y):=v(x, y)$, and get:

$$
v(x, y)+\int_{0}^{x} L(s, y) v(x, s) d s=0, \quad 0 \leq y \leq x,
$$

provided that $-q(x) L(x, y)+2 \dot{K} L(x, y)=0$, which is true because of (4.1.4). Equation (4.4.6) has only the trivial solution by Lemma 4.3.1. Thus $v=0$, and equation (4.1.1) is derived.

We have proved

Lemma 4.4.2. If $L(x, y)$ is twice differentiable continuously or in $L^{2}$-sense then the solution $K(x, y)$ of (1.5.4) solves (4.1.1), (4.1.2) with q given by (4.1.4).

Remark 4.4.3. If a Fredholm equation

$$
(I+A(x)) u=f(x)
$$

in a Banach space $X$ depends on a parameter $x$ continuously in the sense $\lim _{h \rightarrow 0}\|A(x+h)-A(x)\|=$ 0 , $\lim _{h \rightarrow 0}\|f(x+h)-f(x)\|=0$, and at $x=x_{0}$ equation (4.4.7) has $N\left(I+A\left(x_{0}\right)\right)=\{0\}$, where $N(A)=\{u: A u=0\}$, then the solution $u(x)$ exists, is unique, and depends continuously on $x$ in some neighborhood of $x_{0},\left|x-x_{0}\right|<r$. If the data, that is, $A(x)$ and $f(x)$, have $m$ derivatives with respect to $x$, then the solution has the same number of derivatives.

Derivatives are understood in the strong sense for the elements of $X$ and in the operator norm for the operator $A(x)$.

\subsection{Characterization of the class of spectral functions of the Sturm-Liouville operators}

From Theorem 4.4.1 it follows that if (4.2.2) holds and $L(x) \in H_{l o c}^{1}\left(\mathbb{R}_{+}\right)$, then $q \in L_{l o c}^{2}\left(\mathbb{R}_{+}\right)$. Condition (4.2.3) was used only to prove $L \Rightarrow \rho$, so if one starts with a $q \in L_{l o c}^{2}\left(\mathbb{R}_{+}\right)$, then by diagram (1.5.5) one gets $L(x, y)$ by formula (4.3.6), where $L(x) \in H_{l o c}^{1}\left(\mathbb{R}_{+}\right)$. If $(4.2 .3)$ holds, then one gets from $L(x)$ a unique $\rho(\lambda)$.

Recall that assumption $\left.A_{1}\right)$ is (4.2.2). Let $\left.A_{3}\right)$ be the assumption $L(x) \in H_{l o c}^{m+1}\left(\mathbb{R}_{+}\right)$.

Theorem 4.5.1. If $\left.A_{1}\right)$ holds, and $\rho$ is a spectral function of $\ell_{q}, q \in H_{l o c}^{m}\left(\mathbb{R}_{+}\right)$, then assumption $\left.A_{3}\right)$ holds. Conversely, if assumptions $\left.A_{1}\right)$ and $A_{3}$ ) hold, then $\rho$ is a spectral function of $\ell_{q}, q \in H_{l o c}^{m}\left(\mathbb{R}_{+}\right)$.

Proof. If $\left.A_{1}\right)$ holds and $q \in H_{l o c}^{m}\left(\mathbb{R}_{+}\right)$, then $L(x) \in H_{l o c}^{m+1}\left(\mathbb{R}_{+}\right)$by (4.1.4). If $\left.A_{1}\right)$ and $\left.A_{3}\right)$ hold, then $q \in H_{l o c}^{m}\left(\mathbb{R}_{+}\right)$by (1.5.2), because equation (1.5.4) is uniquely solvable, and (1.5.5) holds by Theorem 4.4.1. 


\subsection{Relation to the inverse scattering problem}

Assume in this Section that $q \in L_{1,1}$. Then the scattering data $\mathcal{S}$ are (1.2.17) and the spectral function is $(1.2 .21)$.

Let us show how to get $d \rho$, given $\mathcal{S}$. If $\mathcal{S}$ is given then $s_{j}, k_{j}$ and $J$ are known. If one finds $f(k)$ then $d \rho$ is recovered because

$$
c_{j}=-\frac{4 k_{j}^{2}}{\left[\dot{f}\left(i k_{j}\right)\right]^{2}} \frac{1}{s_{j}}
$$

as follows from (1.2.20) and (1.2.16). To find $f(k)$, consider the Riemann problem

$$
f(k)=S(-k) f(-k), \quad k \in \mathbb{R}, \quad f(\infty)=1,
$$

which can be written as (see (3.4.3)):

$$
\begin{gathered}
f_{0}(k)=S(-k) \frac{w(-k)}{w(k)} f_{0}(-k) \quad \text { if ind } S(k)=-2 J, \\
f_{0}(k)=S(-k) \frac{w(-k)}{w(k)} \frac{k+i \kappa}{k-i \kappa} f_{0}(-k) \quad \text { if ind } S(k)=-2 J-1 .
\end{gathered}
$$

Note that $w(-k)=\frac{1}{w(k)}$ if $k \in \mathbb{R}$. The function $f_{0}(k)$ is analytic in $\mathbb{C}_{+}$and has no zeros in $\mathbb{C}_{+}$, and $f_{0}(-k)$ has similar properties in $\mathbb{C}_{-}$. Therefore problems (4.6.3) and (4.6.4) have unique solutions:

$$
\begin{gathered}
f_{0}(k)=\exp \left\{\frac{1}{2 \pi i} \int_{-\infty}^{\infty} \frac{\log \left[S(-t) w^{-2}(t)\right] d t}{t-k}\right\} \quad \text { if ind } S(k)=-2 J, \quad \operatorname{Im} k>0, \\
f_{0}(k)=\exp \left\{\frac{1}{2 \pi i} \int_{-\infty}^{\infty} \frac{\log \left[S(-t) w^{-2}(t) \frac{t+i \kappa}{t-i \kappa}\right]}{t-k}\right\} d t \\
\quad \text { if } \operatorname{ind} S(k)=-2 J-1, \quad \operatorname{Im} k>0,
\end{gathered}
$$

and

$$
\begin{gathered}
f(k)=f_{0}(k) w(k) \quad \text { if ind } S(k)=-2 J, \quad \operatorname{Im} k>0, \\
f(k)=f_{0}(k) w(k) \frac{k}{k+i \kappa} \quad \text { if ind } S(k)=-2 J-1, \quad \operatorname{Im} k>0 .
\end{gathered}
$$

One can calculate $f(x)$ for $k>0$ by taking $k=k+i 0$ in (4.6.7) or (4.6.8). Thus, to find $d \rho$, given $\mathcal{S}$, one goes through the following steps: 1 ) one finds $\left.J, s_{j}, k_{j}, 1 \leq j \leq J ; 2\right)$ one calculates ind $S(k):=\mathcal{J}$. If $\mathcal{J}=-2 J$, then one calculates $f(k)$ by formulas (4.6.5), (4.6.7), where $w(k)$ is defined in (3.4.3), and $c_{j}$ by formula (4.6.1), and, finally, $d \rho$ by formula (1.2.21).

If $\mathcal{J}=-2 J-1$, then one calculates $f(k)$ by formulas (4.6.6) and (4.6.8), where $\kappa>0$ is an arbitrary number such that $\kappa \neq k_{j}, 1 \leq j \leq J$. If $f(k)$ is found, one calculates $c_{j}$ by formula (4.6.1), and then $d \rho$ by formula (1.2.21). Note that $f_{0}(k)$ in (4.6.6) depends on $\kappa$, but $f(k)$ in (4.6.8) does not.

This completes the description of the step $\mathcal{S} \Rightarrow \rho$.

Let us show how to get $\mathcal{S}$ given $d \rho(\lambda)$.

From formula (1.2.21) one finds $J, k_{j}, c_{j}$ and $|f(k)|$. If $|f(0)| \neq 0$, then $\left|f_{0}(k)\right|=|f(k)|$ if $k \in \mathbb{R}$. Thus, if $|f(0)| \neq 0$, then $\log f_{0}(k)$ is analytic in $\mathbb{C}_{+}$and vanishes at infinity. It can be found in $\mathbb{C}_{+}$from the values of its real part $\log \left|f_{0}(k)\right|$ by Schwarz's formula for the half-plane:

$$
\log f_{0}(k)=\frac{1}{i \pi} \int_{-\infty}^{\infty} \frac{\log \left|f_{0}(t)\right|}{t-k} d t, \quad \operatorname{Im} k>0
$$


If $f(0) \neq 0$, then $f=f_{0} w$, so

$$
f(k)=\exp \left\{\frac{1}{i \pi} \int_{-\infty}^{\infty} \frac{\log \left|f_{0}(t)\right| d t}{t-k}\right\} w(k), \quad \operatorname{Im} k>0 .
$$

If $|f(0)|=0$, then the same formula (4.6.10) remains valid. One can see this because $\frac{f(k)}{w(k)}$ is analytic in $\mathbb{C}_{+}$, has no zeroes in $\mathbb{C}_{+}$, tends to 1 at infinity, and $\left|\frac{f(k)}{w(k)}\right|=|f(k)|$ if $k \in \mathbb{R}$.

Let us summarize the step $d \rho \Rightarrow \mathcal{S}$ : one finds $J, k_{j}, c_{j}$, calculates $f(k)$ by formula (4.6.10), and then $S(k)=\frac{f(-k)}{f(k)}$, and $s_{j}$ are calculated by formula (4.6.1). To calculate $f(k)$ for $k>0$ one takes $k=k+i 0$ in (4.6.10) and gets:

$$
\begin{aligned}
f(k) & =\exp \left\{\frac{1}{i \pi} \int_{-\infty}^{\infty} \frac{\log |f(t)| d t}{t-k}+\log |f(k)|\right\} w(k) \\
& =|f(k)| w(k) \exp \left\{\frac{1}{i \pi} P \int_{-\infty}^{\infty} \frac{\log |f(t)| d t}{t-k}\right\}, \quad k>0 .
\end{aligned}
$$




\section{Chapter 5}

\section{Inverse scattering on half-line}

\subsection{Auxiliary material}

\subsubsection{Transformation operators}

Theorem 5.1.1. If $q \in L_{1,1}$, then there exists a unique operator $I+A$ such that $(1.2 .25)-(1.2 .28)$ hold, and $A(x, y)$ solves the following Goursat problem:

$$
\begin{gathered}
A_{x x}-q(x) A=A_{y y}, 0 \leq x \leq y \leq \infty \\
A(x, x)=\frac{1}{2} \int_{x}^{\infty} q(s) d s \\
\lim _{x+y \rightarrow \infty} A(x, y)=\lim _{x+y \rightarrow \infty} A_{x}(x, y)=\lim _{x+y \rightarrow \infty} A_{y}(x, y)=0 .
\end{gathered}
$$

Proof. Equations (5.1.1) and (5.1.2) are derived similarly to the derivation of the similar equations for $K(x, y)$ in Theorem 4.1.1. Relations (5.1.3) follow from the estimates $(1.2 .26)-(1.2 .27)$, which give more precise information than (5.1.3). Estimates $(1.2 .26)-(1.2 .28)$ can be derived from the Volterra equation (1.2.28) which is solvable by iterations. Equation (1.2.28) can be derived, for example, similarly to the derivation of equation (4.1.8), or by substituting (1.2.25) into (1.2.6).

A detailed derivation of all of the results of Theorem 5.1.1 can be found in $[\mathrm{M}]$.

\subsubsection{Statement of the direct scattering problem on half-axis. Existence and uniqueness of its solution.}

The direct scattering problem on half-line consists of finding the solution $\psi=\psi(r, k)$ to the equation:

$$
\psi^{\prime \prime}+k^{2} \psi-q(r) \psi=0, \quad r>0,
$$

satisfying the boundary conditions at $r=0$ and at $r=\infty$ :

$$
\begin{gathered}
\psi(0)=0, \\
\psi(r)=e^{i \delta} \sin (k r+\delta)+o(1), \quad r \rightarrow+\infty,
\end{gathered}
$$

where $\delta=\delta(k)$ is called the phase shift, and it has to be found. An equivalent formulation of (5.1.6) is:

$$
\psi=\frac{i}{2}\left[e^{-i k r}-S(k) e^{i k r}\right]+o(1), \quad r \rightarrow \infty,
$$


where $S(k)=\frac{f(-k)}{f(k)}=e^{2 i \delta(k)}$. Clearly

$$
\psi(r, k)=\frac{i}{2}[f(r,-k)-S(k) f(r, k)]=a(k) \varphi(r, k), \quad a(k):=\frac{k}{f(k)},
$$

where $\varphi(r, k)$ is defined in (1.2.1), see also (1.2.10). From (5.1.8), (1.2.7) and (5.1.6) one gets

$$
\varphi(r, k)=\frac{|f(k)|}{k} \sin (k r+\delta(k))+o(1), \quad r \rightarrow \infty
$$

Existence and uniqueness of the scattering solution $\psi(r, k)$ follows from (5.1.8) because existence and uniqueness of the regular solution $\varphi(r, k)$ follows from (1.2.1) or from (1.2.9).

\subsubsection{Higher angular momenta.}

If one studies the three-dimensional scattering problem with a spherically-symmetric potential $q(x)=$ $q(r), x \in \mathbb{R}^{3},|x|=r$, then the scattering solution $\psi(r, \alpha, k)$ solves the problem:

$$
\begin{gathered}
{\left[\nabla^{2}+k^{2}-q(r)\right] \psi=0 \text { in } R^{3}} \\
\psi=e^{i k \alpha \cdot x}+A\left(\alpha^{\prime}, \alpha, k\right) \frac{e^{i k r}}{r}+o\left(\frac{1}{r}\right), r:=|x| \rightarrow \infty, \alpha^{\prime}:=\frac{x}{r}, \alpha \in S^{2} .
\end{gathered}
$$

Here $S^{2}$ is the unit sphere in $\mathbb{R}^{3} \alpha \in S^{2}$ is given, $A\left(\alpha^{\prime}, \alpha, k\right)$ is called the scattering amplitude. If $q=q(r)$, then $A\left(\alpha^{\prime}, \alpha, k\right)=A\left(\alpha^{\prime} \cdot \alpha, k\right)$. The converse is a theorem of Ramm [R], p.130. The scattering solution solves the integral equation:

$$
\psi=e^{i k \alpha \cdot x}-\int_{\mathbb{R}^{3}} g(x, y, k) q(y) \psi(y, \alpha, k) d y, \quad g:=\frac{e^{i k|x-y|}}{4 \pi|x-y|} .
$$

It is known that

$$
e^{i k \alpha \cdot x}=\sum_{\ell=0}^{\infty} \frac{4 \pi}{k} i^{\ell} \frac{u_{\ell}(k r)}{r} Y_{\ell}\left(\alpha^{\prime}\right) \overline{Y_{\ell}(\alpha)}, \quad \alpha^{\prime}:=\frac{x}{r}, \quad u_{\ell}:=\sqrt{\frac{\pi r}{2}} J_{\ell+\frac{1}{2}},
$$

$Y_{\ell}(\alpha)$ are orthonormal in $L^{2}\left(S^{2}\right)$ spherical harmonics, $Y_{l}=Y_{\ell m},-\ell \leq m \leq \ell$, and summation over $m$ in (5.1.13) is understood but not shown, and $J_{\ell}(r)$ is the Bessel function.

If $q=q(r)$, then

$$
\psi=\sum_{\ell=0}^{\infty} \frac{4 \pi}{k} i^{\ell} \frac{\psi_{\ell}(r, k)}{r} Y_{\ell}\left(\alpha^{\prime}\right) \overline{Y_{\ell}(\alpha)}
$$

where

$$
\begin{gathered}
\psi_{\ell}^{\prime \prime}+k^{2} \psi_{\ell}-q(r) \psi_{\ell}-\frac{\ell(\ell+1)}{r^{2}} \psi_{\ell}=0, \\
\psi_{\ell}=e^{i \delta_{\ell}} \sin \left(k r-\frac{\ell \pi}{2}+\delta_{\ell}\right)+o(1), \quad r \rightarrow \infty, \\
\psi_{\ell}=O\left(r^{\ell+1}\right), \quad r \rightarrow 0 .
\end{gathered}
$$

Relation (5.1.16) is equivalent to

$$
\psi_{\ell}=\frac{e^{i \frac{\pi}{2}(\ell+1)}}{2}\left[e^{-i k r}-e^{i \pi \ell} S_{\ell} e^{i k r}\right]+o(1), \quad r \rightarrow \infty
$$


similar to (5.1.8), which is (5.1.18) with $\ell=0$. If $q=q(r)$, then the scattering amplitude $A\left(\alpha^{\prime}, \alpha,\right)=$ $A\left(\alpha^{\prime} \cdot \alpha, k\right)$ can be written as

$$
A\left(\alpha^{\prime} \cdot \alpha, k\right)=\sum_{\ell=0}^{\infty} A_{\ell}(k) Y_{\ell}\left(\alpha^{\prime}\right) \overline{Y_{\ell}(\alpha)},
$$

while in the general case $q=q(x)$, one has

$$
A\left(\alpha^{\prime}, \alpha, k\right)=\sum_{\ell=0}^{\infty} A_{l}(\alpha, k) Y_{\ell}\left(\alpha^{\prime}\right) .
$$

If $q=q(r)$ then $S_{\ell}$ in (5.1.18) are related to $A_{\ell}$ in (5.1.19) by the formula

$$
S_{\ell}=1-\frac{k}{2 \pi i} A_{\ell}
$$

In the general case $q=q(x)$, one has a relation between $S$-matrix and the scattering amplitude:

$$
S=I-\frac{k}{2 \pi i} A,
$$

so that (5.1.21) is a consequence of (5.1.22) in the case $q=q(r): S_{\ell}$ are the eigenvalues of $S$ in the eigenbasis of spherical harmonics. Since $S$ is unitary, one has $\left|S_{\ell}\right|=1$, so $S_{\ell}=e^{2 i \delta_{\ell}}$ for some real numbers $\delta_{\ell}$, which are called phase-shifts. These numbers are the same as in (5.1.17) (cf. (5.1.18)). From (5.1.21) one gets

$$
A_{\ell}(k)=\frac{4 \pi}{k} e^{i \delta_{\ell}} \sin \left(\delta_{\ell}\right)
$$

The Green function $g_{\ell}(r, \rho)$, which solves the equation

$$
\left(\frac{d^{2}}{d r^{2}}+k^{2}-\frac{\ell(\ell+1)}{r^{2}}\right) g_{\ell}=-\delta(r-\rho), \quad \frac{\partial g_{\ell}}{\partial r}-i k g_{\ell} \underset{r \rightarrow+\infty}{\rightarrow} 0,
$$

can be written explicitly:

$$
g_{\ell}(r, \rho)=\left\{\begin{array}{llll}
F_{o \ell}^{-1} & \varphi_{o \ell}(k \rho) f_{o \ell}(k r) & r \geq \rho, & F_{o \ell}:=\frac{e^{\frac{i \ell \pi}{2}}}{k^{\ell}}, \\
F_{o \ell}^{-1} & \varphi_{o \ell}(k r) f_{o \ell}(k \rho), & r<\rho, & \varphi_{o \ell}(k r)=\frac{u_{\ell}(k r)}{k^{\ell+1}},
\end{array}\right.
$$

and the function $\psi_{\ell}(r, k)$ solves the equation:

$$
\psi_{\ell}(r, k)=u_{\ell}(k r)-\int_{0}^{\infty} g_{\ell}(r, \rho) q(\rho) \psi_{\ell}(\rho, k) d \rho .
$$

The function $F_{o \ell}(k)$ is the Wronskian $W\left[f_{o \ell}, \varphi_{o \ell}\right], \varphi_{o \ell}(k r)$ is defined in (5.1.25) and $f_{o \ell}$ is the solution to (5.1.15) (with $q=0$ ) with the asymptotics

$$
\begin{aligned}
f_{o \ell}=e^{i k r} & +o(1), \quad r \rightarrow+\infty, \quad f_{o \ell}(k r)=e^{i \frac{(\ell+1) \pi}{2}}\left(u_{\ell}(k r)+i v_{\ell}(k r)\right), \\
v_{\ell} & :=\sqrt{\frac{\pi r}{2}} N_{\ell+\frac{1}{2}}(k r) .
\end{aligned}
$$

Let $\varphi_{\ell}(r, k)$ be the regular solution to (5.1.15) which is defined by the asymptotics as $r \rightarrow 0$ :

$$
\varphi_{\ell}(r, k)=\frac{r^{\ell+1}}{(2 \ell+1) ! !}+o\left(r^{\ell+1}\right), \quad r \rightarrow 0
$$

Then

$$
\begin{aligned}
& \psi_{\ell}(r, k)=a_{\ell}(k) \varphi_{\ell}(r, k), \\
& \varphi_{\ell}(r, k)=\frac{\left|f_{\ell}(0, k)\right|}{k^{\ell+1}} \sin \left(k r-\frac{\ell \pi}{2}+\delta_{\ell}\right)+o(1), r \rightarrow \infty .
\end{aligned}
$$


Lemma 5.1.2. One has:

$$
\sup _{\ell=0,1,2, \ldots}\left|a_{\ell}(k)\right|<\infty
$$

where $k>0$ is a fixed number.

We omit the proof of this lemma.

\subsubsection{Eigenfunction expansion}

We assume that $q \in L_{1,1}$ and $h \in C_{0}^{\infty}\left(\mathbb{R}_{+}\right), \ell h=-h^{\prime \prime}+q(x) h, \lambda=k^{2}$, let $g=\frac{\varphi(x, \sqrt{\lambda}) f(y, \sqrt{\lambda})}{f(\sqrt{\lambda})}, y \geq x \geq 0$, be the resolvent kernel of $\ell:(\ell-\lambda) g=\delta(x-y), g h:=g(\lambda) h:=\int_{0}^{\infty} g(x, y, \lambda) h d y$, and $f_{j}=f\left(y, i k_{j}\right)$. Then $\frac{h}{\lambda}=-g h+\frac{1}{\lambda} \ell g h$. Integrate this with respect to $\lambda \in \mathbb{C}$ over $|\lambda|=N$ and divide by $2 \pi i$ to get

$$
h=-\frac{1}{2 \pi i} \int_{|\lambda|=N} g h d \lambda+\frac{1}{2 \pi i} \int_{|\lambda|=N} \frac{\ell g h}{\lambda} d \lambda:=I_{1}+I_{2} .
$$

The function $g h$ is analytic with respect to $\lambda$ on the complex plane with the cut $(0, \infty)$ except for the points $\lambda=-k_{j}^{2}, 1 \leq j \leq J$, which are simple poles of $g h$, and $\lim _{N \rightarrow \infty} I_{2}=0$, because $|\ell g h|=o(1)$ as $N \rightarrow \infty$. Therefore:

$$
h=\frac{1}{2 \pi i} \int_{0}^{\infty}[g(\lambda+i 0) h-g(\lambda-i 0) h] d \lambda+\sum_{j=1}^{J} \frac{-1}{2 \pi i} \oint_{\left|\lambda+k_{j}^{2}\right|=\delta} g h d \lambda .
$$

One has (cf. (1.2.10)):

$$
\begin{aligned}
& \frac{g(\lambda+i 0)-g(\lambda-i 0)}{2 i}=\varphi(x, k) \frac{f(-k) f(y, k)-f(y,-k) f(k)}{2 i|f(k)|^{2}} \\
& =\frac{k}{|f(k)|^{2}} \varphi(x, k) \varphi(y, k), \quad k=\sqrt{\lambda}>0 .
\end{aligned}
$$

Also

$$
\begin{aligned}
-\frac{1}{2 \pi i} & \oint_{\left|\lambda+k_{j}^{2}\right|=\delta} g h d \lambda=-\operatorname{Res}_{\lambda=-k_{j}^{2}} g h \\
& =-\int_{0}^{\infty} f_{j}(y) h(y) d y \cdot \frac{\varphi\left(x, i k_{j}\right)}{\dot{f}\left(i k_{j}\right)} 2 i k_{j}=s_{j} f_{j}(x) h_{j}, h_{j}:=\int_{0}^{\infty} f_{j} h d y,
\end{aligned}
$$

$s_{j}$ are defined in (1.2.16), and

$$
\varphi\left(x, i k_{j}\right)=\frac{f\left(x, i k_{j}\right)}{f^{\prime}\left(0, i k_{j}\right)}:=\frac{f_{j}(x)}{f^{\prime}\left(0, i k_{j}\right)} .
$$

Therefore

$$
h(x)=\int_{0}^{\infty}\left(\int_{0}^{\infty} \varphi(y, k) h(y) d y\right) \varphi(x, k) \frac{2 k^{2} d k}{\pi|f(k)|^{2}}+\sum_{j=1}^{J} s_{j} f_{j}(x) h_{j} .
$$

This implies (cf. (1.2.21), (1.2.20), (1.2.16)):

$$
\begin{aligned}
\delta(x-y) & =\frac{2}{\pi} \int_{0}^{\infty} \varphi(x, k) \varphi(y, k) \frac{k^{2} d k}{|f(k)|^{2}}+\sum_{j=1}^{J} s_{j} f_{j}(x) f_{j}(y) \\
& =\int_{-\infty}^{\infty} \varphi(x, \sqrt{\lambda}) \varphi(y, \sqrt{\lambda}) d \rho(\lambda) .
\end{aligned}
$$

We have proved the eigenfunction expansion theorem for $h \in C_{0}^{\infty}\left(\mathbb{R}_{+}\right)$. Since this set is dense in $L^{2}\left(\mathbb{R}_{+}\right)$, one gets the theorem for $h \in L^{2}\left(\mathbb{R}_{+}\right)$. 
Theorem 5.1.3. If $q \in L_{1,1}$, then (5.1.33) holds for any $h \in L^{2}\left(\mathbb{R}_{+}\right)$and the integrals converge in $L^{2}\left(\mathbb{R}_{+}\right)$sense. Parseval's equality is:

$$
\|h\|_{L^{2}\left(\mathbb{R}_{+}\right)}^{2}=\sum_{j=1}^{J} s_{j}\left|h_{j}\right|^{2}+\frac{2}{\pi} \int_{0}^{\infty}|\widetilde{h}(k)|^{2} \frac{k^{2} d k}{|f(k)|^{2}}, \quad \widetilde{h}:=\int_{0}^{\infty} h(y) \varphi(y, k) d y .
$$

\subsection{Statement of the inverse scattering problem on the half- line. Uniqueness theorem}

In Section 1.3 the statement of the ISP is given. Let us prove the uniqueness theorem.

Theorem 5.2.1. If $q_{1}, q_{2} \in L_{1,1}$ generate the same data (1.2.17), then $q_{1}=q_{2}$.

Proof. We prove that the data (1.2.17) determine uniquely $I(k)$, and this implies $q_{1}=q_{2}$ by Theorem 3.1.2.

Claim 1. If (1.2.17) is given, then $f(k)$ is uniquely determined.

Assume there are $f_{1}(k)$ and $f_{2}(k)$ corresponding to the data (1.2.17). Then

$$
\frac{f_{1}(k)}{f_{2}(k)}=\frac{f_{1}(-k)}{f_{2}(-k)}, \quad-\infty<k<\infty .
$$

The left-hand side of (5.2.1) is analytic in $\mathbb{C}_{+}$and tends to 1 as $|k| \rightarrow \infty, k \in \mathbb{C}_{+}$, and the right-hand side of (5.2.1) is analytic in $\mathbb{C}_{-}$and tends to 1 as $|k| \rightarrow \infty, k \in \mathbb{C}_{-}$. By analytic continuation $\frac{f_{1}(k)}{f_{2}(k)}$ is an analytic function in $\mathbb{C}$, which tends to 1 as $|k| \rightarrow \infty, k \in \mathbb{C}$. Thus, by Liouville theorem, $\frac{f_{1}(k)}{f_{2}(k)}=1$, so $f_{1}=f_{2}$.

Claim 2. If (1.2.17) is given, then $f^{\prime}(0, k)$ is uniquely defined.

Assume there are $f_{1}^{\prime}(0, k)$ and $f_{2}^{\prime}(0, k)$ corresponding to (1.2.17). By the Wronskian relation (1.2.11), taking into account that $f_{1}(k)=f_{2}(k):=f(k)$ by Claim 1 , one gets

$$
\left[f_{1}^{\prime}(0, k)-f_{2}^{\prime}(0, k)\right] f(-k)-\left[f_{1}^{\prime}(0,-k)-f_{2}^{\prime}(0,-k)\right] f(k)=0 .
$$

Denote $w(k):=f_{1}^{\prime}(0, k)-f_{2}^{\prime}(0, k)$. Then:

$$
\frac{w(k)}{f(k)}=\frac{w(-k)}{f(-k)}, \quad k \in \mathbb{R} .
$$

The function $\frac{w(k)}{f(k)}$ is analytic in $\mathbb{C}_{+}$and tends to zero as $|k| \rightarrow \infty, k \in \mathbb{C}_{+}$, and $\frac{w(-k)}{f(-k)}$ has similar properties in $\mathbb{C}_{-}$. It follows that $\frac{w(k)}{f(k)}=0$, so $f_{1}^{\prime}(0, k)=f_{2}^{\prime}(0, k)$. Let us check that $\frac{w(k)}{f(k)}$ is analytic in $\mathbb{C}_{+}$. One has to check that $w\left(i k_{j}\right)=0$. This follows from(1.2.16): if $f(k), s_{j}$ and $k_{j}$ are given, then $f^{\prime}\left(0, i k_{j}\right)$ are uniquely determined.

Let us check that $w(k) \rightarrow 0$ as $|k| \rightarrow \infty, k \in \mathbb{C}_{+}$. Using (3.1.5) it is sufficient to check that $A(0,0)$ is uniquely determined by $f(k)$, because the integral in (3.1.5) tends to zero as $|k| \rightarrow \infty, k \in \mathbb{C}_{+}$by the Riemann-Lebesgue lemma. From (1.5.16), integrating by parts one gets:

$$
f(k)=1-\frac{A(0,0)}{i k}-\frac{1}{i k} \int_{0}^{\infty} e^{i k y} A_{y}(0, y) d y .
$$

Thus

Claim 2 is proved.

$$
\left.A(0,0)=-\lim _{k \rightarrow \infty}[i k f(k)-1)\right]
$$

Thus, Theorem 5.2.1 is proved. 


\subsection{Reconstruction procedure}

This procedure is described in (1.5.10).

Let us derive equation (1.5.13). Our starting point is formula (5.1.34):

$$
\int_{0}^{\infty} \varphi(x, k) \varphi(y, k) \frac{2 k^{2} d k}{\pi|f(k)|^{2}}+\sum_{j=1}^{J} s_{j} f_{j}(x) f_{j}(y)=0 \quad y>x \geq 0 .
$$

From (1.2.10) and (1.2.25) one gets:

$$
\begin{aligned}
\frac{k \varphi(x, k)}{|f(k)|} & =\sin (k x+\delta)+\int_{x}^{\infty} A(x, y) \sin (k y+\delta) d y \\
& =(I+A) \sin (k x+\delta), \quad \delta=\delta(k) .
\end{aligned}
$$

Apply to (5.3.1) operator $(I+A)^{-1}$, acting on the functions of $y$, and get:

$$
\frac{2}{\pi} \int_{0}^{\infty} \frac{k \varphi(x, k)}{|f(k)|} \sin (k y+\delta) d k+\sum_{j=1}^{J} s_{j} f_{j}(x) e^{-k_{j} y}=0, \quad y>x \geq 0 .
$$

From (5.3.2), (5.3.3), and (1.2.25) with $k=i k_{j}$, one gets:

$$
\begin{aligned}
(I+A)\left(\frac{2}{\pi}\right. & \left.\int_{0}^{\infty} \sin (k x+\delta) \sin (k y+\delta) d k\right) \\
& +(I+A) \sum_{j=1}^{J} s_{j} e^{-k_{j}(x+y)}=0, y>x \geq 0 .
\end{aligned}
$$

One has

$$
\begin{aligned}
& \frac{2}{\pi} \int_{0}^{\infty} \sin (k x+\delta) \sin (k y+\delta) d k=\frac{1}{\pi} \int_{0}^{\infty} \cos [k(x-y)] d k \\
& \quad-\frac{1}{\pi} \int_{0}^{\infty} \cos [k(x+y)+2 \delta(k)] d k=\delta(x, y)-\frac{1}{2 \pi} \int_{-\infty}^{\infty}\left(e^{2 i \delta(k)}-1\right) e^{i k(x+y)} d k \\
& \quad=\delta(x-y)+\frac{1}{2 \pi} \int_{-\infty}^{\infty}[1-S(k)] e^{i k(x+y)} d k
\end{aligned}
$$

From (1.5.11), (5.3.4) and (5.3.5) one gets (1.5.13). By continuity equation (1.5.13), derived for $y>x \geq$ 0 , remains valid for $y \geq x \geq 0$.

Theorem 5.3.1. If $q \in L_{1,1}$ and $F$ is defined by (1.5.11) then equation (1.5.13) has a solution in $L^{1}\left(\mathbb{R}_{x}\right) \cap L^{\infty}\left(\mathbb{R}_{x}\right), \mathbb{R}_{x}:=[x, \infty)$, for any $x \geq 0$, and this solution is unique.

Let us outline the steps of the proof.

Step 1. If $q \in L_{1,1}$, then $F(x)$, defined by (1.5.11) satisfies the following estimates:

$$
|F(2 x)| \leq c \sigma(x)|,| F(2 x)+A(x, x)|\leq c \sigma(x),| F^{\prime}(2 x)-\frac{q(x)}{4} \mid \leq c \sigma^{2}(x),
$$

where $\sigma(x)$ is defined in (1.2.26), and

$$
\begin{gathered}
\|F\|_{L^{2}\left(\mathbb{R}_{+}\right)}+\|F\|_{L^{1}\left(\mathbb{R}_{+}\right)}+\|F\|_{L^{\infty}\left(\mathbb{R}_{+}\right)}+\left\|x F^{\prime}(x)\right\|_{L^{1}\left(\mathbb{R}_{+}\right)}<\infty, \\
\int_{0}^{\infty} \int_{0}^{\infty}|F(s+y)|^{2} d s d y<\infty .
\end{gathered}
$$


Step 2. Equation

$$
\left(I+F_{x}\right) h:=h(y)+\int_{x}^{\infty} h(s) F(s+y) d s=0, \quad y \geq x \geq 0
$$

is of Fredholm type in $L^{1}\left(\mathbb{R}_{x}\right), L^{2}\left(\mathbb{R}_{x}\right)$ and in $L^{\infty}\left(\mathbb{R}_{x}\right)$. It has only the trivial solution $h=0$.

Using estimates (5.3.6) - (5.3.8) and the criteria of compactness in $L^{p}\left(\mathbb{R}_{x}\right), p=1,2, \infty$, one checks that $F_{x}$ is compact in these spaces for any $x \geq 0$. The space $L^{1} \cap L^{\infty} \subset L^{2}$ because $\|h\|_{2} \leq\|h\|_{1}\|h\|_{\infty}$, where $\|h\|_{p}:=\|h\|_{L^{p}\left(\mathbb{R}_{x}\right)}$. We need the following lemma:

Lemma 5.3.2. Let $h$ solve (5.3.9). If $h \in L^{1}:=L^{1}\left(\mathbb{R}_{x}\right)$, then $h \in L^{2}:=L^{2}\left(\mathbb{R}_{x}\right)$. If $h \in L^{1}$, then $h \in L^{\infty}$. If $h \in L^{2}$, then $h \in L^{\infty}$.

Proof. If $h$ solves (5.3.9), then $\|h\|_{\infty} \leq\|h\|_{1} \sup _{y \geq 2 x}|F(y)| \leq c(x)\|h\|_{1}<\infty$, where $c(x) \rightarrow 0$ as $x \rightarrow \infty$. Also $\|h\|_{2}^{2} \leq \int_{x}^{\infty} d y \sigma^{2}\left(\frac{x+y}{2}\right)\|h\|_{1}^{2} \leq c_{1}(x)\|h\|_{1}^{2}<\infty, c_{1}(x) \rightarrow 0$ as $x \rightarrow \infty$. So the first claim is proved. Also $\|h\|_{1} \leq\|h\|_{1} \sup _{s \geq x} \int_{x}^{\infty}|F(s+y)| d y=c_{2}(x)\|h\|_{1}, c_{2}(x) \rightarrow 0$ as $x \rightarrow \infty$. If $h \in L^{2}$, then $\|h\|_{\infty} \leq\|h\|_{2} \sup _{y \geq x}\left(\int_{x}^{\infty}|F(s+y)|^{2} d s\right)^{\frac{1}{2}}=c_{3}(x)\|h\|_{2}, c_{3}(x) \rightarrow 0$ as $x \rightarrow \infty$.

Lemma 5.3.3. If $h \in L^{1}$ solves (5.3.9) and $x \geq 0$, then $h=0$.

Proof. By Lemma 5.3.2, $h \in L^{2} \cap L^{\infty}$. It is sufficient to give a proof assuming $x=0$. The function $F(x)$ is real-valued, so one can assume that $h$ is real-valued. Multiply $(5.3 .9)$ by $h$ and integrate over $(x, \infty)$ to get

$$
\begin{gathered}
\|h\|^{2}+\frac{1}{2 \pi} \int_{-\infty}^{\infty}[1-S(k)] \widetilde{h}^{2}(k) d k+\sum_{j=1}^{J} s_{j}\left(\int_{x}^{\infty} e^{-k_{j} s} h(s) d s\right)^{2}=0, \\
\tilde{h}:=\int_{x}^{\infty} e^{i k s} h(s) d s,
\end{gathered}
$$

where $\|h\|=\|h\|_{L^{2}\left(\mathbb{R}_{+}\right)}$one gets $\int_{-\infty}^{\infty} \widetilde{h}^{2}(k) d k=0$. Also, $\left|\frac{1}{2 \pi} \int_{-\infty}^{\infty} S(k) \widetilde{h}^{2}(k) d k\right| \leq \frac{1}{2 \pi} \int_{-\infty}^{\infty}|\widetilde{h}(k)|^{2} d k=$ $\|h\|^{2}$. Therefore (5.3.10) implies $0=h_{j}:=\int_{x}^{\infty} h e^{-k_{j} s} d s, 1 \leq j \leq J$, and

$$
(\widetilde{h}, \widetilde{h})=(\widetilde{h}, S(-k) \widetilde{h}(-k))
$$

where $(\widetilde{h}, \widetilde{g}):=\int_{-\infty}^{\infty} \widetilde{h}(k) \overline{\widetilde{g}(k)} d k$. Since $h$ is real-valued, one has $\overline{\widetilde{h}}(-k)$. The unitarity of $S$ implies $S^{-1}(k)=S(-k)=\overline{S(k)}, k \in \mathbb{R}$, and $\|S(-k) \widetilde{h}(-k)\|=\|\widetilde{h}(-k)\|$. Because of (5.3.11), one has equality sign in the Cauchy inequality $\left(\widetilde{h}, S(-k) \widetilde{h}(-k) \leq\|\widetilde{h}\|^{2}\right.$. This means that $\widetilde{h}(k)=S(-k) \widetilde{h}(-k)$, and (1.2.17) implies

$$
\frac{\widetilde{h}(k)}{f(k)}=\frac{\widetilde{h}(-k)}{f(-k)}, \quad k \in \mathbb{R} .
$$

Because $h_{j}=0$, one has $\widetilde{h}\left(i k_{j}\right)=0$, and if $f(0) \neq 0$, then $\frac{\widetilde{h}(k)}{f(k)}$ is analytic in $\mathbb{C}_{+}$and vanishes as $|k| \rightarrow \infty$, $k \in \infty, k \in \mathbb{C}_{+}$. Also $\frac{\widetilde{h}(-k)}{f(-k)}$ is analytic in $\mathbb{C}_{-}$and vanishes as $|k| \rightarrow \infty, k \in \mathbb{C}_{-}$. Therefore, by analytic continuation, $\frac{\widetilde{h}(k)}{f(k)}$ is analytic in $\mathbb{C}$ and vanishes as $|k| \rightarrow \infty$. By Liouville theorem, $\frac{\widetilde{h}(k)}{f(k)}=0$, so $\widetilde{h}(k)=0$ and $h=0$. If $f(0)=0$, then, by Theorem 3.1.3, $f(k)=i k \widetilde{A}_{1}(k), \widetilde{A}_{1}(0) \neq 0$, and the above argument works.

Because $F_{x}$ is compact in $L^{2}\left(\mathbb{R}_{x}\right)$, the Fredholm alternative is applicable to (5.3.9), and Lemma 5.3.3 implies that (1.5.13) has a solution in $L^{2}\left(\mathbb{R}_{x}\right)$ for any $x \geq 0$, and this solution is unique. Note that the free term in (1.5.13) is $-F(x+y)$, and this function of $y$ belongs to $L^{2}\left(\mathbb{R}_{x}\right)$ (cf. (5.3.8)). Because $F_{x}$ is compact in $L^{1}\left(\mathbb{R}_{x}\right)$, Lemma 5.3.3 and Lemma 5.3.2 imply existence and uniqueness of the solution 
to (1.5.13) in $L^{1}\left(\mathbb{R}_{x}\right)$ for any $x \geq 0$, and $F(x+y) \in L^{1}\left(\mathbb{R}_{x}\right)$ for any $x \geq 0$. Note that the solution to (1.5.13) in $L^{1}\left(\mathbb{R}_{x}\right)$ is the same as its solution in $L^{2}\left(\mathbb{R}_{x}\right)$. This is established by the argument used in the proof of Lemma 5.3.2.

We give a method for the derivation of the estimates (5.3.6) - (5.3.8). Estimate (5.3.8) is an immediate consequence of the first estimate (5.3.6). Indeed,

$$
\begin{aligned}
\int_{0}^{\infty} & \int_{0}^{\infty}|F(s+y)|^{2} d s d y \leq\left(\int_{0}^{\infty} \max _{s \geq 0}|F(s+y)| d y\right)^{2} \\
& \leq c\left(\int_{0}^{\infty} \int_{\frac{y}{2}}^{\infty}|q| d t d y\right)^{2} \leq c\left(\int_{0}^{\infty} t|q(t)| d t\right)^{2}<\infty .
\end{aligned}
$$

Let us prove the first estimate (5.3.6). Put in (1.5.13) $x=y$ :

$$
A(x, x)+\int_{x}^{\infty} A(x, s) F(s+x) d x+F(2 x)=0 .
$$

Thus

$$
|F(2 x)| \leq|A(x, x)|+\int_{x}^{\infty}|A(x, s) F(s+x)| d x .
$$

From (1.2.26) and (5.3.14) one gets

$$
\begin{aligned}
|F(2 x)| & \leq c \sigma(x)+c \int_{x}^{\infty} \sigma\left(\frac{x+s}{2}\right)|F(s+x)| d s \\
& \leq c \sigma(x)+c \sigma(x) \int_{x}^{\infty}|F(s+x)| d s \leq c \sigma(x),
\end{aligned}
$$

where $c=$ const $>0$ stands for various constants and we have used the estimate

$$
\sup _{x \geq 0} \int_{x}^{\infty}|F(s+x)| d s \leq \int_{0}^{\infty}|F(t)| d t=c<\infty .
$$

This estimate can be derived from (1.2.26). Write (1.5.13) as

$$
A(x, z-x)+\int_{z}^{\infty} A(x, t+x-z) F(t) d t+F(z)=0, \quad z \geq 2 x \geq 0 .
$$

Let us prove that equation (5.3.16) is uniquely solvable for $F$ in $L^{p}\left(\mathbb{R}_{N}\right), p=\infty, p=1$ for all $x \geq \frac{N}{2}$, where $N$ is a sufficiently large number. In fact, we prove that the operator in (5.3.16) has small norm in $L^{p}\left(\mathbb{R}_{N}\right)$ if $N$ is sufficiently large. Its norm in $L^{\infty}\left(\mathbb{R}_{N}\right)$ is not more than

$$
\begin{aligned}
\sup _{z \geq N} & \int_{N}^{\infty}|A(x, t+x-z)| d t \leq c \int_{x+\frac{t-N}{2}}^{\infty}|q(s)| d s \\
& \leq c \int_{N}^{\infty} d t \int_{t}^{\infty}|q(s)| d s=c \int_{N}^{\infty}(s-N)|q(s)| d s<1
\end{aligned}
$$

because $q \in L_{1,1}$. We have used estimate (1.2.26) above. The function $A(x, y) \in L^{\infty}\left(\mathbb{R}_{N}\right)$, so our claim is proved for $p=\infty$. Consider the case $p=1$. One has the following upper estimate for the norm of the operator in (5.3.16) in $L^{1}\left(\mathbb{R}_{N}\right): \sup _{t \geq N} \int_{N}^{t}|A(x, t+x-z)| d z \leq \sup _{t \geq N} \int_{0}^{t-N}|A(x, x+v)| d v \leq$ $\int_{0}^{\infty} d v \int_{x+\frac{v}{2}}^{\infty}|q| d s=2 \int_{x}^{\infty}(s-x)|q| d s \rightarrow 0$ as $x \rightarrow \infty$. Also $\int_{N}^{\infty}|A(x, z-x)| d z<\infty$. Thus equation (5.3.16) is uniquely solvable in $L^{1}\left(\mathbb{R}_{N}\right)$ for all $x \geq \frac{N}{2}$ if $N$ is sufficiently large. In order to finish the proof of the first estimate (5.3.6) it is sufficient to prove that $\|F\|_{L^{\infty}(0, N)} \leq c<\infty$. This estimate is 
obvious for $F_{d}(x)$ (cf. (1.5.11)). Let us prove it for $F_{s}(x)$. Using (3.4.3), (3.4.5), (3.4.5'), (3.4.6), one gets

$$
1-S(k)=\frac{[f(k)-f(-k)](k+i \kappa)}{f_{0}(k) w(k) k}=[\widetilde{A}(k)-\widetilde{A}(-k)](1+\widetilde{b}(k))(1+\widetilde{g})\left(1+\frac{i \kappa}{k}\right),
$$

where all the Fourier transforms are taken of $W^{1,1}\left(\mathbb{R}_{+}\right)$functions. Thus, one can conclude that $F_{s}(x) \in$ $L^{\infty}\left(\mathbb{R}_{+}\right)$if one can prove that $I:=\frac{\widetilde{A}(k)-\widetilde{A}(-k)}{k}$ is the Fourier transform of $L^{\infty}\left(\mathbb{R}_{+}\right)$function. One has $I=\int_{0}^{\infty} d y A(y) \frac{e^{i k y}-e^{-i k y}}{k}$ and

$$
\begin{aligned}
\int_{-\infty}^{\infty} e^{i k x} I(k) d k & =\int_{0}^{\infty} d y A(y) \int_{-\infty}^{\infty} \frac{e^{i k(x+y)}-e^{i k(x-y)}}{k} \\
& =\int_{0}^{\infty} d y A(y) i \pi[1-\operatorname{sgn}(x-y)]=2 i \pi \int_{x}^{\infty} d y A(y) .
\end{aligned}
$$

From (1.2.26) it follows that $\int_{x}^{\infty} A(y) d y \in L^{\infty}\left(\mathbb{R}_{+}\right)$. We have proved that $\|F\|_{L^{\infty}\left(\mathbb{R}_{+}\right)}+\|F\|_{L^{1}\left(\mathbb{R}_{+}\right)}<\infty$. Differentiate (5.3.13) to get

$$
\begin{aligned}
2 F^{\prime}(2 x) & +\dot{A}(x, x)-A(x, x) F(2 x)+\int_{x}^{\infty} A_{x}(x, s) F(s+x) d s \\
& +\int_{x}^{\infty} A(x, s) F^{\prime}(s+x) d s=0, \quad \dot{A}:=\frac{d A(x, x)}{d x}
\end{aligned}
$$

or

$$
F^{\prime}(2 x)=\frac{q(x)}{4}+A(x, x) F(2 x)-\frac{1}{2} \int_{x}^{\infty}\left[A_{x}(x, s)-A_{s}(x, s)\right] F(s+x) d s .
$$

One has $\int_{0}^{\infty} x|q| d x<\infty, \int_{0}^{\infty} x|A(x, x)||F(2 x)| d x \leq \sup _{x \geq 0}(x|A(x, x)|) \cdot \int_{0}^{\infty}|F(2 x)| d x \leq c$. Let us check that $I:=\int_{0}^{\infty} x\left|\int_{x}^{\infty}\left[A_{x}(x, s)-A_{s}(x, s)\right] F(s+x) d s\right| d x<\infty$. Use (1.2.27) and get $I \leq c \int_{0}^{\infty} x \sigma(x) \int_{x}^{\infty} \sigma$ $\left(\frac{x+s}{2}\right)|F(s+x)| d s d x \leq c \int_{0}^{\infty} \sigma(x) d x \int_{0}^{\infty}|F(y)| d y \cdot \sup _{x \geq 0, s \geq x} x \sigma\left(\frac{x+s}{2}\right) \leq c<\infty$. The desired estimate is derived.

The third estimate (5.3.6), $\left|F^{\prime}(2 x)-\frac{q(x)}{4}\right| \leq c \sigma^{2}(x)$ follows from (5.3.20) because $|A(x, x)| \leq$ $c \sigma(x),|F(2 x)| \leq c \sigma(x)$, and $\int_{x}^{\infty}\left|A_{x}(x, s)-A_{s}(x, s)\right|\left|F(s+x) d s \leq c \sigma(x) \int_{x}^{\infty} \sigma\left(\frac{x+s}{2}\right)\right| F(s+x) \mid d s \leq$ $c \sigma^{2}(x) \int_{0}^{\infty}|F(s+x)| d s \leq c \sigma^{2}(x)$. The estimate $|F(2 x)+A(x, x)| \leq c \sigma(x)$ follows similarly from (5.3.13) and (1.2.26). Theorem 5.3.1 is proved.

\subsection{Invertibility of the steps of the reconstruction procedure}

The reconstruction procedure is (1.5.10). 1. The step $\mathcal{S} \Rightarrow F$ is done by formula (1.5.11).

To do the step $F \Rightarrow \mathcal{S}$, one takes $x \rightarrow-\infty$ in (1.5.11) and finds $s_{j}, k_{j}$, and $J$. Thus $F_{d}(x)$ is found and $F_{s}=F-F_{d}$ is found. From $F_{s}(x)$ one finds $1-S(k)$ by the inverse Fourier transform. So $S(k)$ is found and the data $\mathcal{S}$ (see (1.2.17)) is found

2. The step $F \Rightarrow A$ is done by solving equation (1.5.13). By Theorem 5.3.1 this equation is uniquely solvable in $L^{1}\left(\mathbb{R}_{x}\right) \cap L^{\infty}\left(\mathbb{R}_{x}\right)$ for all $x \geq 0$ if $q \in L_{1,1}$, that is, if $F$ came from $\mathcal{S}$ corresponding to $q \in L_{1,1}$.

To do the step $A \Rightarrow F$, one finds $f(k)=1+\int_{0}^{\infty} A(0, y) e^{i k y} d y$, then the numbers $i k_{j}$, the zeros of $f(k)$ in $\mathbb{C}_{+}$, the number $J, 1 \leq j \leq J$, and $S(k)=\frac{f(-k)}{f(k)}$. The numbers $s_{j}$ are found by formula (1.2.16), where

$$
f^{\prime}\left(0, i k_{j}\right)=-k_{j}-A(0,0)+\int_{0}^{\infty} A_{x}(0, y) e^{-k_{j} y} d y .
$$

Thus $A \Rightarrow \mathcal{S}$ and $\mathcal{S} \Rightarrow F$ by formula (1.5.11). 
We also give a direct way to do the step $A \Rightarrow F$.

Write equation (1.5.13) with $z=x+y, v=s+y$, as

$$
\left(I+B_{x}\right) F:=F(z)+\int_{z}^{\infty} A(x, v+x-z) F(v) d v=-A(x, z-x), \quad z \geq 2 x \geq 0 .
$$

The norm of the operator $B_{x}$ in $L_{2 x}^{1}$ is estimated as follows:

$$
\left\|B_{x}\right\| \leq \sup _{v>0} \int_{0}^{v}|A(x, v+x-z)| d z \leq c \sup _{v>0} \int_{0}^{v} \sigma\left(x+\frac{v-z}{2}\right) d z \leq c \int_{x}^{\infty} \sigma(t) d t,
$$

where $\sigma(x)=\int_{x}^{\infty}|q(t)| d t$ and estimate (1.2.26) was used. If $x_{0}$ is sufficiently large then $\left\|B_{x}\right\|<1$ for $x \geq x_{0}$ because $\int_{x}^{\infty} \sigma(t) d t \rightarrow 0$ as $x \rightarrow \infty$ if $q \in L_{1,1}$. Therefore equation (5.4.2) is uniquely solvable in $L_{2 x}^{1}$ for all $x \geq x_{0}$ (by the contraction mapping principle), and so $F(z)$ is uniquely determined for all $z \geq 2 x_{0}$.

Now rewrite (5.4.2) as

$$
F(z)+\int_{z}^{2 x_{0}} A(x, v+x-z) F(v) d v=-A(x, z-x)-\int_{2 x_{0}}^{\infty} A(x, v+x-z) F(v) d v .
$$

This is a Volterra equation for $F(z)$ on the finite interval $\left(0,2 x_{0}\right)$. It is uniquely solvable since its kernel is a continuous function. One can put $x=0$ in (5.4.4) and the kernel $A(0, v-z)$ is a continuous function of $v$ and $z$, and the right-hand side of (5.4.4) at $x=0$ is a continuous function of $z$. Thus $F(z)$ is uniquely recovered for all $z \geq 0$ from $A(x, y), y \geq x \geq 0$. Step $S \Rightarrow F$ is done.

3. The step $A \Rightarrow q$ is done by formula (1.5.12). The converse step $q \Rightarrow A$ is done by solving Volterra equation (1.2.28), or, equivalently, the Goursat problem (5.1.1) - (5.1.3).

We have proved:

Theorem 5.4.1. If $q \in L_{1,1}$ and $\mathcal{S}$ are the corresponding data (1.2.17), then each step in (1.5.10) is invertible. In particular, the potential obtained by the procedure (1.5.10) equals to the original potential $q$.

Remark 5.4.2. If $q \in L_{1,1}$ and $A_{q}:=A_{q}(x, y)$ is the solution to (1.2.28), then $A_{q}$ satisfies equation (1.5.13) and, by the uniqueness of its solution, $A_{q}=A$, where $A$ is the function obtained by the scheme (1.5.10). Therefore, the q obtained by (1.5.10) equals to the original $q$.

Remark 5.4.3. One can verify directly that the solution $A(x, y)$ to (1.5.13) solves the Goursat problem (5.1.1) - (5.1.3). This is done as in Section 4.4, Step 3. Therefore $q(x)$, obtained by the scheme (1.5.10), generates the same $A(x, y)$ which was obtained at the second step of this scheme, and therefore this $q$ generates the original scattering data.

Remark 5.4.4. The uniqueness Theorem 5.2.1 does not imply that if one starts with a $q_{0} \in L_{1,1}$, computes the corresponding scattering data (1.2.17), and applies inversion scheme (1.5.10), then the $q$ obtained by this scheme is equal to $q_{0}$. Logically it is possible that this q generates data $\mathcal{S}_{1}$ which generate by the scheme(1.5.10) potential $q_{1}$, etc. To close this loop one has to check that $q=q_{0}$. This is done in Theorem 5.4.1, because $q_{0}=-2 \frac{d A(x, x)}{d x}=q(x)$.

\subsection{Characterization of the scattering data}

In this Section we give a necessary and sufficient condition for the data (1.2.17) to be the scattering data corresponding to $q \in L_{1,1}$. In Section 5.7 we give such conditions on $\mathcal{S}$ for $q$ to be compactly supported, or $q \in L^{2}\left(\mathbb{R}_{+}\right)$. 
Theorem 5.5.1. If $q \in L_{1,1}$, then the following conditions hold: 1) (1.2.23); 2) $k_{j}>0, s_{j}>0$, $1 \leq j \leq J, S(k)=\overline{S(-k)}=S^{-1}(k), k \geq 0, S(\infty)=1$; 3) (5.3.7) hold. Conversely, if $\mathcal{S}$ satisfies conditions 1) - 3), then $\mathcal{S}$ corresponds to a unique $q \in L_{1,1}$.

Proof. The necessity of conditions 1) - 3) has been proved in Theorem 5.3.1. Let us prove the sufficiency. If conditions 1) - 3) hold, then the scheme (1.5.10) yields a unique potential, as was proved in Remark 5.4.2. Indeed, equation (1.5.13) is of Fredholm type in $L^{1}\left(\mathbb{R}_{x}\right)$ for every $x \geq 0$ if $F$ satisfies (5.3.7). Moreover, equation (5.3.9) has only the trivial solution if conditions 1) - 3) hold. Every solution to (5.3.9) in $L^{1}\left(\mathbb{R}_{x}\right)$ is also a solution in $L^{2}\left(\mathbb{R}_{x}\right)$ and in $L^{\infty}\left(\mathbb{R}_{x}\right)$, and the proof of the uniqueness of the solution to (5.3.9) under the conditions 1) - 3) goes as in Theorem 5.3.1. The role of $f(k)$ is played by the unique solution of the Riemann problem:

$$
f_{+}(k)=S(-k) f_{-}(k), \quad k \in \mathbb{R},
$$

which consists of finding two functions $f_{+}(k)$ and $f_{-}(k)$, satisfying (5.5.1), such that $f_{+}$is an analytic function in $\mathbb{C}_{+}, f_{+}\left(i k_{j}\right)=0, \dot{f}_{+}\left(i k_{j}\right) \neq 0,1 \leq j \leq J, f_{+}(\infty)=1$, and $f_{-}(k)$ is an analytic function in $\mathbb{C}_{-}$such that $f_{-}\left(-i k_{j}\right)=0, \dot{f}_{-}\left(-i k_{j}\right) \neq 0,1 \leq j \leq J, f_{-}(\infty)=1$, and $f_{+}(0)=0$ if ind $S(k)=-2 J-1$, $f_{+}(0) \neq 0$ if ind $S(k)=-2 J$. Existence of a solution to (5.5.1) follows from the non-negativity of ind $S(-k)=-$ ind $S(k)$. Uniqueness of the solution to the above problem is proved as follows. Denote $f_{+}(k):=f(k)$ and $f_{-}(k)=f(-k)$. Assume that $f_{1}$ and $f_{2}$ solve the above problem. Then (5.5.1) implies

$$
\frac{f_{1}(k)}{f_{2}(k)}=\frac{f_{1}(-k)}{f_{2}(-k)}, k \in \mathbb{R}, \quad f_{1}\left(i k_{j}\right)=f_{2}\left(i k_{j}\right)=0, \quad \dot{f}_{1}\left(i k_{j}\right) \neq 0, \quad \dot{f}_{2}\left(i k_{j}\right) \neq 0, \quad f_{1}(\infty)=f_{2}(\infty)=1 .
$$

The function $\frac{f_{1}(k)}{f_{2}(k)}$ is analytic in $\mathbb{C}_{+}$and tends to 1 at infinity in $\mathbb{C}_{+}$, The function $\frac{f_{1}(-k)}{f_{2}(-k)}$ is analytic in $\mathbb{C}_{-}$and tends to 1 at infinity in $\mathbb{C}_{-}$. Both functions agree on $\mathbb{R}$. Thus $\frac{f_{1}(k)}{f_{2}(k)}$ is analytic in $\mathbb{C}$ and tends to 1 at infinity. Therefore $f_{1}(k)=f_{2}(k)$. To complete the proof we need to check that $q$, obtained by (1.5.10), belongs to $L_{1,1}$. In other words, that $q=-2 \frac{d A(x, x)}{d x} \in L_{1,1}$. To prove this, use (5.3.19) and (5.3.20). It is sufficient to check that $F^{\prime}(2 x) \in L_{1,1}, A(x, x) F(2 x) \in L_{1,1}$ and $\int_{x}^{\infty}\left[A_{x}(x, s)-A_{x}(x, s)\right] F(s+x) d s \in$ $L_{1,1}$. The first inclusion follows from $\left\|x F^{\prime}\right\|_{L^{1}\left(\mathbb{R}_{+}\right)}<\infty$. Let us prove that $\lim _{x \rightarrow \infty}[x F(x)]=0$. One has $\int_{0}^{x} s F^{\prime} d s=x F(x)-\int_{0}^{x} F d s$. Because $x F^{\prime} \in L^{1}\left(\mathbb{R}_{+}\right)$and $F \in L^{1}\left(\mathbb{R}_{+}\right)$it follows that the limit $c_{0}:=\lim _{x \rightarrow \infty} x F$ exists. This limit has to be zero: if $F=\frac{c_{0}}{x}+o\left(\frac{1}{x}\right)$ as $x \rightarrow \infty$, and $c_{0} \neq 0$, then $F \notin L^{1}\left(\mathbb{R}_{+}\right)$. Now $\int_{0}^{\infty} x|F(2 x) A(x, x)| d x \leq c \int_{0}^{\infty}|A(x, x)| d x<\infty$. The last inequality follows from (5.3.13): $\quad$ since $F(2 x) \in L^{1}\left(\mathbb{R}_{+}\right)$it is sufficient to check that $\int_{x}^{\infty} A(x, s) F(s+x) d s \in L^{1}\left(\mathbb{R}_{+}\right)$. One has $\int_{0}^{\infty} d x \int_{x}^{\infty}|A(x, s)||F(s+x)| d s \leq \int_{0}^{\infty} d x \sigma_{F}(2 x) \int_{x}^{\infty}|A(x, s)| d s \leq c$. Here

$$
\sigma_{F}(x):=\sup _{y \geq x}|F(y)|, \quad \sigma_{F} \in L^{1}\left(\mathbb{R}_{+}\right) .
$$

Note that $\lim _{x \rightarrow \infty} x \sigma_{F}(x)=0$ because $\sigma_{F}(x)$ is monotonically decreasing and belongs to $L^{1}\left(\mathbb{R}_{+}\right)$.

\subsection{A new equation of Marchenko-type}

The basic result of this Section is:

Theorem 5.6.1. Equation

$$
F(y)+A(y)+\int_{-\infty}^{\infty} A(t) F(t+y) d t=A(-y), \quad-\infty<y<\infty,
$$

holds, where $A(y):=A(0, y), A(y)=0$ for $y<0, A(x, y)$ is defined in (1.2.25) and $F(x)$ is defined in (1.5.11). 
Proof. Take the Fourier transform of (5.6.1) in the sense of distributions and get:

$$
\widetilde{F}(\xi)+\widetilde{A}(\xi)+\widetilde{A}(-\xi) \widetilde{F}(\xi)=\widetilde{A}(-\xi),
$$

where, by (1.5.11),

$$
\widetilde{F}(\xi)=1-S(-\xi)+2 \pi \sum_{j=1}^{J} s_{j} \delta\left(\xi_{i}+i k_{j}\right) .
$$

Use (1.5.16), the equation $S(\xi) f(\xi)=f(-\xi)$, add 1 to both sides of (5.3.8), and get:

$$
f(\xi)+f(-\xi) \widetilde{F}(\xi)=f(-\xi) .
$$

From (5.6.3) and (5.6.4) one gets:

$$
f(\xi)=f(-\xi)\left[S(-\xi)-2 \pi \sum_{j=1}^{J} s_{j} \delta\left(\xi+i k_{j}\right)\right]=f(\xi)-2 \pi \sum_{j=1}^{J} s_{j} \delta\left(\xi+i k_{j}\right) f(-\xi)=f(\xi),
$$

where the equation $\delta\left(\xi+i k_{j}\right) f(-\xi)=0$ was used. This equation holds because $f\left(i k_{j}\right)=0$, and the product $\delta\left(\xi+i k_{j}\right) f(-\xi)$ makes sense because $f(\xi)$ is analytic in $\mathbb{C}_{+}$. Equation (5.6.5) holds obviously, and since each of our steps was invertible, equation (5.6.1) holds.

Remark 5.6.2. Equation (5.6.1) has a unique solution $A(y)$, such that $A(y) \in L^{1}\left(\mathbb{R}_{+}\right)$and $A(y)=0$ for $y<0$.

Proof. Equation (5.6.1) for $y>0$ is identical with (1.5.13) because $A(-y)=0$ for $y>0$. Equation (1.5.13) has a solution in $L^{1}\left(\mathbb{R}_{+}\right)$and this solution is unique, see Theorem 5.3.1. Thus, equation (5.6.1) cannot have more than one solution, because every solution $A(y) \in L^{1}\left(\mathbb{R}_{+}\right), A(y)=0$ for $y<0$, of (5.6.1) solves (1.5.13), and (1.5.13) has no more than one solution. On the other hand, the solution $A(y) \in L^{1}\left(\mathbb{R}_{+}\right)$of (1.5.13) does exist, is unique, and solves (5.6.1), as was shown in the proof of Theorem 5.6.1. This proves Remark 5.6.2.

\subsection{Inequalities for the transformation operators and applica- tions}

\subsubsection{Inequalities for $A$ and $F$}

The scattering data (1.2.17) satisfy the following conditions:

A) $k_{j}, s_{j}>0, S(-k)=\overline{S(k)}=S^{-1}(k), k \in \mathbb{R}, S(\infty)=1$,

B) $\mathcal{J}:=\operatorname{ind} S(k):=\frac{1}{2 \pi} \int_{-\infty}^{\infty} d \log S(k)$ is a nonpositive integer,

C) $F \in L^{p}, p=1$ and $p=\infty, x F^{\prime} \in L^{1}, L^{p}:=L^{p}(0, \infty)$.

If one wants to study the characteristic properties of the scattering data, that is, a necessary and sufficient condition on these data to guarantee that the corresponding potential belongs to a prescribed functional class, then conditions A) and B) are always necessary for a real-valued $q$ to be in $L_{1,1}$, the usual class in the scattering theory, or in some other class for which the scattering theory is constructed, and a condition of the type C) determines actually the class of potentials $q$. Conditions A) and B) are consequences of the selfadjointness of the Hamiltonian, finiteness of its negative spectrum, and of the unitarity of the $S$ - matrix. Our aim is to derive some inequalities for $F$ and $A$ from equation (1.5.13). This allows one to describe the set of $q$, defined by (1.5.13).

Let us assume:

$$
\sup _{y \geq x}|F(y)|:=\sigma_{F}(x) \in L^{1}, \quad F^{\prime} \in L_{1,1} .
$$


The function $\sigma_{F}$ is monotone decreasing, $|F(x)| \leq \sigma_{F}(x)$. Equation (1.5.13) is of Fredholm type in $L_{x}^{p}:=L^{p}(x, \infty) \forall x \geq 0$ and $p=1$. The norm of the operator $F:=F_{x}$ in (1.5.13) can be estimated :

$$
\left\|F_{x}\right\| \leq \int_{x}^{\infty} \sigma_{F}(x+y) d y \leq \sigma_{1 F}(2 x), \quad \sigma_{1 F}(x):=\int_{x}^{\infty} \sigma_{F}(y) d y .
$$

Therefore (1.5.13) is uniquely solvable in $L_{x}^{1}$ for any $x \geq x_{0}$ if

$$
\sigma_{1 F}\left(2 x_{0}\right)<1
$$

This conclusion is valid for any $F$ satisfying (5.7.3), and conditions A), B), and C) are not used. Assuming (5.7.3) and (5.7.1) and taking $x \geq x_{0}$, let us derive inequalities for $A=A(x, y)$. Define

$$
\sigma_{A}(x):=\sup _{y \geq x}|A(x, y)|:=\|A\| .
$$

From (1.5.13) one gets:

$$
\sigma_{A}(x) \leq \sigma_{F}(2 x)+\sigma_{A}(x) \sup _{y \geq x} \int_{x}^{\infty} \sigma_{F}(s+y) d s \leq \sigma_{F}(2 x)+\sigma_{A}(x) \sigma_{1 F}(2 x) .
$$

Thus, if (5.7.3) holds, then

$$
\sigma_{A}(x) \leq c \sigma_{F}(2 x), \quad x \geq x_{0} .
$$

By $c>0$ different constants depending on $x_{0}$ are denoted. Let

$$
\sigma_{1 A}(x):=\|A\|_{1}:=\int_{x}^{\infty}|A(x, s)| d s .
$$

Then (1.5.13) yields $\sigma_{1 A}(x) \leq \sigma_{1 F}(2 x)+\sigma_{1 A}(x) \sigma_{1 F}(2 x)$. So

$$
\sigma_{1 A}(x) \leq c \sigma_{1 F}(2 x), \quad x \geq x_{0} .
$$

Differentiate (1.5.13) with respect to $x$ and $y$ and get:

$$
\left(I+F_{x}\right) A_{x}(x, y)=A(x, x) F(x+y)-F^{\prime}(x+y), \quad y \geq x \geq 0,
$$

and

$$
A_{y}(x, y)+\int_{x}^{\infty} A(x, s) F^{\prime}(s+y) d s=-F^{\prime}(x+y), \quad y \geq x \geq 0 .
$$

Denote

$$
\sigma_{2 F}(x):=\int_{x}^{\infty}\left|F^{\prime}(y)\right| d y, \quad \sigma_{2 F}(x) \in L^{1} .
$$

Then, using (5.7.7) and (5.7.4), one gets

$$
\left\|A_{y}\right\|_{1} \leq \int_{x}^{\infty}\left|F^{\prime}(x+y)\right| d y+\sigma_{1 A}(x) \sup _{s \geq x} \int_{x}^{\infty}\left|F^{\prime}(s+y)\right| d y \leq \sigma_{2 F}(2 x)\left[1+c \sigma_{1 F}(2 x)\right] \leq c \sigma_{2 F}(2 x)
$$

and using (5.7.6) one gets:

$$
\left\|A_{x}\right\|_{1} \leq A(x, x) \sigma_{1 F}(2 x)+\sigma_{2 F}(2 x)+\left\|A_{x}\right\|_{1} \sigma_{1 F}(2 x),
$$

so

$$
\left\|A_{x}\right\|_{1} \leq c\left[\sigma_{2 F}(2 x)+\sigma_{1 F}(2 x) \sigma_{F}(2 x)\right] .
$$


Let $y=x$ in (1.5.13), then differentiate (1.5.13) with respect to $x$ and get:

$$
\dot{A}(x, x)=-2 F^{\prime}(2 x)+A(x, x) F(2 x)-\int_{x}^{\infty} A_{x}(x, s) F(x+s) d s-\int_{x}^{\infty} A(x, s) F^{\prime}(s+x) d s .
$$

From (5.7.4), (5.7.5), (5.7.10) and (5.7.11) one gets:

$$
|\dot{A}(x, x)| \leq 2\left|F^{\prime}(2 x)\right|+c \sigma_{F}^{2}(2 x)+c \sigma_{F}(2 x)\left[\sigma_{2 F}(2 x)+\sigma_{1 F}(2 x) \sigma_{F}(2 x)\right]+c \sigma_{F}(2 x) \sigma_{2 F}(2 x) .
$$

Thus,

$$
x|\dot{A}(x, x)| \in L^{1},
$$

provided that $x F^{\prime}(2 x) \in L^{1}, x \sigma_{F}^{2}(2 x) \in L^{1}$, and $x \sigma_{F}(2 x) \sigma_{2 F}(2 x) \in L^{1}$. Assumption (5.7.1) implies $x F^{\prime}(2 x) \in L^{1}$. If $\sigma_{F}(2 x) \in L^{1}$, and $\sigma_{F}(2 x)>0$ decreases monotonically, then $x \sigma_{F}(x) \rightarrow 0$ as $x \rightarrow \infty$. Thus $x \sigma_{F}^{2}(2 x) \in L^{1}$, and $\sigma_{2 F}(2 x) \in L^{1}$ because $\int_{0}^{\infty} d x \int_{x}^{\infty}\left|F^{\prime}(y)\right| d y=\int_{0}^{\infty}\left|F^{\prime}(y)\right| y d y<\infty$, due to (5.7.1). Thus, (5.7.1) implies (5.7.4), (5.7.5), (5.7.8), (5.7.9), and (5.7.12), while (5.7.12) and (1.5.13) imply $q \in \widetilde{L}_{1,1}$ where $\widetilde{L}_{1,1}=\left\{q: q=\bar{q}, \int_{x_{0}}^{\infty} x|q(x)| d x<\infty\right\}$, and $x_{0} \geq 0$ satisfies (5.7.3).

Let us assume now that (5.7.4), (5.7.5), (5.7.9), and (5.7.10) hold, where $\sigma_{F} \in L^{1}$ and $\sigma_{2 F} \in L^{1}$ are some positive monotone decaying functions (which have nothing to do now with the function $F$, solving equation (1.5.13)), and derive estimates for this function $F$. Let us rewrite (1.5.13) as:

$$
F(x+y)+\int_{x}^{\infty} A(x, s) F(s+y) d s=-A(x, y), \quad y \geq x \geq 0 .
$$

Let $x+y=z, s+y=v$. Then,

$$
F(z)+\int_{z}^{\infty} A(x, v+x-z) F(v) d v=-A(x, z-x), \quad z \geq 2 x .
$$

From (5.7.15) one gets:

$$
\sigma_{F}(2 x) \leq \sigma_{A}(x)+\sigma_{F}(2 x) \sup _{z \geq 2 x} \int_{z}^{\infty}|A(x, v+x-z)| d v \leq \sigma_{A}(x)+\sigma_{F}(2 x)\|A\|_{1} .
$$

Thus, using (5.7.5) and (5.7.3), one obtains:

$$
\sigma_{F}(2 x) \leq c \sigma_{A}(x)
$$

Also from (5.7.15) it follows that:

$$
\begin{aligned}
\sigma_{1 F}(2 x) & :=\|F\|_{1}:=\int_{2 x}^{\infty}|F(v)| d v \\
& \leq \int_{2 x}^{\infty}|A(x, z-x)| d z+\int_{2 x}^{\infty} \int_{z}^{\infty}|A(x, v+x-z) \| F(v)| d v d z \\
& \leq\|A\|_{1}+\|F\|_{1}\|A\|_{1} \\
& \text { so } \\
& \sigma_{1 F}(2 x) \leq c \sigma_{1 A}(x) .
\end{aligned}
$$

From (5.7.6) one gets:

$$
\int_{x}^{\infty}\left|F^{\prime}(x+y)\right| d y=\sigma_{2 F}(2 x) \leq c \sigma_{A}(x) \sigma_{1 A}(x)+\left\|A_{x}\right\|+c\left\|A_{x}\right\|_{1} \sigma_{1 A}(x) .
$$

Let us summarize the results: 
Theorem 5.7.1. If $x \geq x_{0}$ and (5.7.1) hold, then one has:

$$
\begin{aligned}
& \sigma_{A}(x) \leq c \sigma_{F}(2 x), \quad \sigma_{1 A}(x) \leq c \sigma_{1 F}(2 x), \quad\left\|A_{y}\right\|_{1} \leq \sigma_{2 F}(2 x)\left(1+c \sigma_{1 F}(2 x)\right), \\
& \left\|A_{x}\right\|_{1} \leq c\left[\sigma_{2 F}(2 x)+\sigma_{1 F}(2 x) \sigma_{F}(2 x)\right] .
\end{aligned}
$$

Conversely, if $x \geq x_{0}$ and

$$
\sigma_{A}(x)+\sigma_{1 A}(x)+\left\|A_{x}\right\|_{1}+\left\|A_{y}\right\|_{1}<\infty
$$

then

$$
\begin{aligned}
& \sigma_{F}(2 x) \leq c \sigma_{A}(x), \quad \sigma_{1 F}(2 x) \leq c \sigma_{1 A}(x), \\
& \sigma_{2 F}(x) \leq c\left[\sigma_{A}(x) \sigma_{1 A}(x)+\left\|A_{x}\right\|_{1}\left(1+\sigma_{1 A}(x)\right)\right] .
\end{aligned}
$$

In the next section we replace the assumption $x \geq x_{0}>0$ by $x \geq 0$. The argument in this case is based on the Fredholm alternative.

\subsubsection{Characterization of the scattering data revisited}

First, let us give necessary and sufficient conditions on $\mathcal{S}$ for $q$ to be in $L_{1,1}$. These conditions are known ([M], [R], [R9], Section 5.5) but we give a short new argument. We assume throughout that conditions A), B), and C) hold. These conditions are known to be necessary for $q \in L_{1,1}$. Indeed, conditions A) and B) are obvious, and C) is proved in Theorem 5.7.1 and Theorem 5.7.4. Conditions A), B), and C) are also sufficient for $q \in L_{1,1}$. Indeed if they hold, then we prove that equation (1.5.13) has a unique solution in $L_{x}^{1}$ for all $x \geq 0$. This was proved in Theorem 5.3.1, but we give another proof.

Theorem 5.7.2. If $A), B$ ), and $C$ ) hold, then (1.5.13) has a solution in $L_{x}^{1}$ for any $x \geq 0$ and this solution is unique.

Proof. Since $F_{x}$ is compact in $L_{x}^{1}, \forall x \geq 0$, by the Fredholm alternative it is sufficient to prove that

$$
\left(I+F_{x}\right) h=0, \quad h \in L_{x}^{1},
$$

implies $h=0$. Let us prove it for $x=0$. The proof is similar for $x>0$. If $h \in L^{1}$, then $h \in L^{\infty}$ because $\|h\|_{\infty} \leq\|h\|_{L^{1} \sigma_{F}}(0)$. If $h \in L^{1} \cap L^{\infty}$, then $h \in L^{2}$ because $\|h\|_{L^{2}}^{2} \leq\|h\|_{L^{\infty}}\|h\|_{L^{1}}$. Thus, if $h \in L^{1}$ and solves (5.7.22), then $h \in L^{2} \cap L^{1} \cap L^{\infty}$.

Denote $\widetilde{h}=\int_{0}^{\infty} h(x) e^{i k x} d x, h \in L^{2}$. Then,

$$
\int_{-\infty}^{\infty} \widetilde{h}^{2} d k=0
$$

Since $F(x)$ is real-valued, one can assume $h$ real-valued. One has, using Parseval's equation:

$$
0=\left(\left(I+F_{0}\right) h, h\right)=\frac{1}{2 \pi}\|\widetilde{h}\|^{2}+\frac{1}{2 \pi} \int_{-\infty}^{\infty}[1-S(k)] \widetilde{h}^{2}(k) d k+\sum_{j=1}^{J} s_{j} h_{j}^{2}, \quad h_{j}:=\int_{0}^{\infty} e^{-k_{j} x} h(x) d x .
$$

Thus, using (5.7.23), one gets

$$
h_{j}=0,1 \leq j \leq J, \quad(\widetilde{h}, \widetilde{h})=(S(k) \widetilde{h}, \widetilde{h}(-k)),
$$

where we have used real-valuedness of $h$, i.e. $\widetilde{h}(-k)=\widetilde{h}(k), \forall k \in R$.

Thus, $(\widetilde{h}, \widetilde{h})=(\widetilde{h}, S(-k) \widetilde{h}(-k))$, where A) was used. Since $\|S(-k)\|=1$, one has $\|\widetilde{h}\|^{2}=\mid(\widetilde{h}$, $S(-k) \widetilde{h}(-k)) \mid \leq\|\widetilde{h}\|^{2}$, so the equality sign is attained in the Cauchy inequality. Therefore, $\widetilde{h}(k)=$ $S(-k) \widetilde{h}(-k)$. 
By condition B), the theory of Riemann problem guarantees existence and uniqueness of an analytic in $\mathbb{C}_{+}:=\{k: \operatorname{Im} k>0\}$ function $f(k):=f_{+}(k), f\left(i k_{j}\right)=0, \dot{f}\left(i k_{j}\right) \neq 0,1 \leq j \leq J, f(\infty)=1$, such that

$$
f_{+}(k)=S(-k) f_{-}(k), \quad k \in \mathbb{R},
$$

and $f_{-}(k)=f(-k)$ is analytic in $\mathbb{C}_{-}:=\{k: \operatorname{Im} k<0\}, f_{-}(\infty)=1$ in $\mathbb{C}_{-}, f_{-}\left(-i k_{j}\right)=0, \dot{f}_{-}\left(-i k_{j}\right) \neq 0$. Here the property $S(-k)=S^{-1}(k), \forall k \in \mathbb{R}$ is used.

One has

$$
\psi(k):=\frac{\widetilde{h}(k)}{f(k)}=\frac{\widetilde{h}(-k)}{f(-k}, \quad k \in \mathbb{R}, \quad h_{j}:=\widetilde{h}\left(i k_{j}\right)=0, \quad 1 \leq j \leq J .
$$

The function $\psi(k)$ is analytic in $\mathbb{C}_{+}$and $\psi(-k)$ is analytic in $\mathbb{C}_{-}$, they agree on $\mathbb{R}$, so $\psi(k)$ is analytic in $\mathbb{C}$. Since $f(\infty)=1$ and $\widetilde{h}(\infty)=0$, it follows that $\psi \equiv 0$.

Thus, $\widetilde{h}=0$ and, consequently, $h(x)=0$, as claimed. Theorem 5.7.2 is proved.

The unique solution to equation (1.5.14) satisfies the estimates given in Theorem 5.7.1. In the proof of Theorem 5.7.1 the estimate $x|\dot{A}(x, x)| \in L^{1}\left(x_{0}, \infty\right)$ was established. So, by (1.5.13), $x q \in L^{1}\left(x_{0}, \infty\right)$.

The method developed in Section 5.7.1 gives accurate information about the behavior of $q$ near infinity. An immediate consequence of Theorem 5.7.1 and Theorem 5.7.2 is:

Theorem 5.7.3. If $A), B)$, and $C$ ) hold, then $q$, obtained by the scheme (1.5.10) belongs to $L_{1,1}\left(x_{0}, \infty\right)$.

Investigation of the behavior of $q(x)$ on $\left(0, x_{0}\right)$ requires additional argument. Instead of using the contraction mapping principle and inequalities, one has to use the Fredholm theorem, which says that $\left\|\left(I+F_{x}\right)^{-1}\right\| \leq c$ for any $x \geq 0$, where the operator norm is taken for $F_{x}$ acting in $L_{x}^{p}, p=1$ and $p=\infty$, and the constant $c$ does not depend on $x \geq 0$.

Such an analysis yields:

Theorem 5.7.4. If and only if $A), B$ ), and $C$ ) hold, then $q \in L_{1,1}$.

Proof. It is sufficient to check that Theorem 5.7.1 holds with $x \geq 0$ replacing $x \geq x_{0}$. To get (5.7.4) with $x_{0}=0$, one uses (1.5.14) and the estimate:

$$
\|A(x, y)\| \leq\left\|\left(I+F_{x}\right)^{-1}||\right\| F(x+y)\left\|\leq c \sigma_{F}(2 x), \quad\right\| \cdot \|=\sup _{y \geq x}|\cdot|, x \geq 0,
$$

where the constant $c>0$ does not depend on $x$. Similarly:

$$
\|A(x, y)\|_{1} \leq c \sup _{s \geq x} \int_{x}^{\infty}|F(s+y)| d y \leq c \sigma_{1 F}(2 x), \quad x \geq 0 .
$$

From (5.7.6) one gets:

$$
\begin{aligned}
& \left\|A_{x}(x, y)\right\|_{1} \leq c\left[\left\|F^{\prime}(x+y)\right\|_{1}+A(x, x)\|F(x+y)\|_{1}\right] \\
& \leq c \sigma_{2 F}(2 x)+c \sigma_{F}(2 x) \sigma_{1 F}(2 x), \quad x \geq 0 .
\end{aligned}
$$

From (5.7.7) one gets:

$$
\left\|A_{y}(x, y)\right\|_{1} \leq c\left[\sigma_{2 F}(2 x)+\sigma_{1 F}(2 x) \sigma_{2 F}(2 x)\right] \leq \sigma_{2 F}(2 x) .
$$

Similarly, from (5.7.11) and (5.7.24) - (5.7.27) one gets (5.7.12). Then one checks (5.7.13) as in the proof of Theorem 5.7.1. Consequently Theorem 5.7.1 holds with $x_{0}=0$. Theorem 5.7.4 is proved. 


\subsubsection{Compactly supported potentials}

In this Section necessary and sufficient conditions are given for $q \in L_{1,1}^{a}:=\{q: q=\bar{q}, q=0$ if $x>$ $\left.a, \int_{0}^{a} x|q| d x<\infty\right\}$. Recall that the Jost solution is:

$$
f(x, k)=e^{i k x}+\int_{x}^{\infty} A(x, y) e^{i k y} d y, \quad f(0, k):=f(k) .
$$

Lemma 5.7.5. If $q \in L_{1,1}^{a}$, then $f(x, k)=e^{i k x}$ for $x>a, A(x, y)=0$ for $y \geq x \geq a, F(x+y)=0$ for $y \geq x \geq a(c f(1.5 .13))$, and $F(x)=0$ for $x \geq 2 a$.

Thus, (1.5.13) with $x=0$ yields $A(0, y):=A(y)=0$ for $x \geq 2 a$. The Jost function

$$
f(k)=1+\int_{0}^{2 a} A(y) e^{i k y} d y, \quad A(y) \in W^{1,1}(0, a),
$$

is an entire function of exponential type $\leq 2 a$, that is, $|f(k)| \leq c e^{2 a|k|}, k \in \mathbb{C}$, and $S(k)=f(-k) / f(k)$ is a meromorphic function in $\mathbb{C}$. In (5.7.30) $W^{l, p}$ is the Sobolev space, and the inclusion (5.7.30) follows from Theorem 5.7.1.

Let us formulate the assumption D):

$D)$ the Jost function $f(k)$ is an entire function of exponential type $\leq 2 a$.

Theorem 5.7.6. Assume A), B), C) and D). Then $q \in L_{1,1}^{a}$. Conversely, if $q \in L_{1,1}^{a}$, then A),B), C) and D) hold.

Necessity. If $q \in L_{1,1}$, then A), B) and C) hold by Theorem 5.7.4, and D) is proved in Lemma 5.7.5. The necessity is proved.

Sufficiency. If A), B) and C) hold, then $q \in L_{1,1}$. One has to prove that $q=0$ for $x>a$. If D) holds, then from the proof of Lemma 5.7.5 it follows that $A(y)=0$ for $y \geq 2 a$.

We claim that $F(x)=0$ for $x \geq 2 a$.

If this is proved, then (1.5.13) yields $A(x, y)=0$ for $y \geq x \geq a$, and so $q=0$ for $x>a$ by (1.5.13).

Let us prove the claim.

Take $x>2 a$ in (1.5.12). The function $1-S(k)$ is analytic in $\mathbb{C}_{+}$except for $J$ simple poles at the points $i k_{j}$. If $x>2 a$ then one can use the Jordan lemma and the residue theorem and get:

$$
F_{s}(x)=\frac{1}{2 \pi} \int_{-\infty}^{\infty}[1-S(k)] e^{i k x} d k=-i \sum_{j=1}^{J} \frac{f\left(-i k_{j}\right)}{\dot{f}\left(i k_{j}\right)} e^{-k_{j} x}, \quad x>2 a .
$$

Since $f(k)$ is entire, the Wronskian formula

$$
f^{\prime}(0, k) f(-k)-f^{\prime}(0,-k) f(k)=2 i k
$$

is valid on $\mathbb{C}$, and at $k=i k_{j}$ it yields:

$$
f^{\prime}\left(0, i k_{j}\right) f\left(-i k_{j}\right)=-2 k_{j}
$$

because $f\left(i k_{j}\right)=0$. This and (5.7.31) yield

$$
F_{s}(x)=\sum_{j=1}^{J} \frac{2 i k_{j}}{f^{\prime}\left(0, i k_{j}\right) \dot{f}\left(i k_{j}\right)} e^{-k_{j} x}=-\sum_{j=1}^{J} s_{j} e^{-k_{j} x}=-F_{d}(x), \quad x>2 a .
$$

Thus, $F(x)=F_{s}(x)+F_{d}(x)=0$ for $x>2 a$. The sufficiency is proved.

Theorem 5.7.6 is proved.

In $[\mathrm{M}]$ a condition on $\mathcal{S}$, which guarantees that $q=0$ for $x>a$, is given under the assumption that there is no discrete spectrum, that is $F=F_{s}$. 


\subsubsection{Square integrable potentials}

Let us introduce conditions (5.7.32) - (5.7.34)

$$
\begin{gathered}
2 i k\left[f(k)-1+\frac{Q}{2 i k}\right] \in L^{2}(\mathbb{R}):=L^{2}, \quad Q:=\int_{0}^{\infty} q d s, \\
k\left[1-S(k)+\frac{Q}{i k}\right] \in L^{2}, \\
k\left[|f(k)|^{2}-1\right] \in L^{2} .
\end{gathered}
$$

Theorem 5.7.7. If A), B), C), and any one of the conditions (5.7.32)-(5.7.34) hold, then $q \in L^{2}(\mathbb{R})$. Proof. We refer to $[\mathrm{R}]$ for the proof. 


\section{Chapter 6}

\section{Inverse scattering problem with fixed-energy phase shifts as the data}

\subsection{Introduction}

In Subsection 5.1.3 the scattering problem for spherically symmetric $q$ was formulated, see (5.1.15) (5.1.17). The $\delta_{\ell}$ are the fixed-energy $(k=$ const $>0)$ phase shifts. Define

$$
L_{r} \varphi:=\left[r^{2} \frac{\partial^{2}}{\partial r^{2}}+r^{2}-r^{2} q(r)\right] \varphi:=L_{0 r} \varphi-r^{2} q(r) \varphi
$$

where $\varphi=\varphi_{\ell}(r)$ is a regular solution to

$$
L_{r} \varphi_{\ell}=\ell(\ell+1) \varphi_{\ell}
$$

such that

$$
\varphi_{\ell}=u_{\ell}+\int_{0}^{r} K(r, \rho) u_{\ell}(\rho) \rho^{-2} d \rho, \quad K(r, 0)=0,
$$

and $u_{l}=\sqrt{\frac{\pi r}{2}} J_{\ell+\frac{1}{2}}(r), J_{\ell}(r)$ is the Bessel function. In (6.1.3) $K(r, \rho)$ is the transformation kernel, $I+K$ is the transformation operator. In (6.1.2) we assume that $k=1$ without loss of generality. The $\varphi_{\ell}$ is uniquely defined by its behavior near the origin:

$$
\varphi_{\ell}(r)=\frac{r^{\ell+1}}{(2 \ell+1) ! !}+o\left(r^{\ell+1}\right), \quad r \rightarrow 0 .
$$

For $u_{\ell}$ we will use the known formula ([GR, 8.411.8]):

$$
\gamma_{\ell} u_{\ell}:=2^{\ell} \Gamma(\ell+1) u_{\ell}(r)=r^{\ell+1} \int_{-1}^{1}\left(1-t^{2}\right)^{\ell} e^{i r t} d t
$$

where $\Gamma(z)$ is the gamma-function.

The inverse scattering problem with fixed-energy phase shifts $\left\{\delta_{\ell}\right\}_{\ell=0,1,2 \ldots}$ as the data consists of finding $q(r)$ from these data. We assume throughout this chapter that $q(r)$ is a real-valued function, $q(r)=0$ for $r>a$,

$$
\int_{0}^{a} r^{2}|q(r)|^{2} d r<\infty
$$

Conditions (6.1.6) imply that $q \in L^{2}\left(B_{a}\right), B_{a}:=\left\{x: x \in \mathbb{R}^{3},|x| \leq a\right\}$. 
In the literature there are books $[\mathrm{CS}]$ and $[\mathrm{N}]$ where the so called Newton-Sabatier (NS) theory is presented, and many papers were published on this theory, which attempts to solve the above inverse scattering problem with fixed-energy phase shifts as the data. In Section 6.4 it is proved that the NS theory is fundamentally wrong and is not an inversion method. The main results of this Chapter are Theorems 6.2.2, 6.3.1, 6.5.1, and the proof of the fact that the Newton-Sabatier theory is fundamentally wrong in the sense that its foundations are wrong.

\subsection{Existence and uniqueness of the transformation operators independent of angular momentum}

The existence and uniqueness of $K(r, \rho)$ in (6.1.3) we prove by deriving a Goursat problem for it, and investigating this problem. Substitute (6.1.3) into (6.1.2), drop index $\ell$ for notational simplicity and get

$$
\begin{aligned}
0=-r^{2} q(r) u+\left(r^{2}-r^{2} q(r)\right) \int_{0}^{r} K(r, \rho) u & \rho^{-2} d \rho \\
& -\int_{0}^{r} K(r, \rho) \rho^{-2} L_{0 \rho} u d \rho+r^{2} \partial_{r}^{2} \int_{0}^{r} K(r, \rho) u \rho^{-2} d \rho .
\end{aligned}
$$

We assume first that $K(r, \rho)$ is twice continuously differentiable with respect to its variables in the region $0<r<\infty, \quad 0<\rho \leq r$. This assumption requires extra smoothness of $q(r), \quad q(r) \in$ $C^{1}(0, a)$. If $q(r)$ satisfies condition $((6.1 .6))$, then equation (6.2.7) below has to be understood in the sense of distributions. Eventually we will work with an integral equation (6.2.34) (see below) for which assumption ((6.1.6)) suffices.

Note that

$$
\int_{0}^{r} K(r, \rho) \rho^{-2} L_{0 \rho} u d \rho=\int_{0}^{r} L_{0 \rho} K(r, \rho) u \rho^{-2} d \rho+K(r, r) u_{r}-K_{\rho}(r, r) u
$$

provided that

$$
K(r, 0)=0
$$

We assume (6.2.3) to be valid. Denote

$$
\dot{K}:=\frac{d K(r, r)}{d r}
$$

Then

$$
\begin{aligned}
& r^{2} \partial_{r}^{2} \int_{0}^{r} K(r, \rho) u \rho^{-2} d \rho=\dot{K} u+K(r, r) u_{r}-\frac{2}{r} K(r, r) u+ \\
& K_{r}(r, r) u+r^{2} \int_{0}^{r} K_{r r}(r, \rho) u \rho^{-2} d \rho .
\end{aligned}
$$

Combining (6.2.1) - (6.2.5) and writing again $u_{\ell}$ in place of $u$, one gets

$$
\begin{aligned}
0=\int_{0}^{r}\left[L_{r} K(r, \rho)-L_{0 \rho} K(r, \rho)\right] u_{\ell}(\rho) \rho^{-2} d \rho+u_{\ell}(r)\left[-r^{2} q(r)+\dot{K}-\right. & \\
& \left.\frac{2 K_{r}(r, r)}{r}+K_{r}(r, r)+K_{\rho}(r, r)\right], \quad \forall r>0, \quad \ell=0,1,2, \ldots
\end{aligned}
$$

Let us prove that (6.2.6) implies:

$$
L_{r} K(r, \rho)=L_{0 \rho} K(r, \rho), \quad 0<\rho \leq r,
$$




$$
q(r)=\frac{2 \dot{K}}{r^{2}}-\frac{2 K(r, r)}{r}=\frac{2}{r} \frac{d}{d r} \frac{K(r, r)}{r} .
$$

This proof requires a lemma.

Lemma 6.2.1. Assume that $\rho f(\rho) \in L^{1}(0, r)$ and $\rho A(\rho) \in L^{1}(0, r)$. If

$$
0=\int_{0}^{r} f(\rho) u_{\ell}(\rho) d \rho+u_{\ell}(r) A(r) \quad \forall \ell=0,1,2, \ldots
$$

then

$$
f(\rho) \equiv 0 \text { and } A(r)=0 .
$$

Proof. Equations (6.2.9) and (6.1.5) imply:

$$
\begin{gathered}
0=\int_{-1}^{1} d t\left(1-t^{2}\right)^{\ell}\left(\frac{d}{i d t}\right)^{\ell} \int_{0}^{r} d \rho \rho f(\rho) e^{i \rho t}+ \\
r A(r) \int_{-1}^{1}\left(1-t^{2}\right)^{\ell}\left(\frac{d}{i d t}\right)^{\ell} e^{i r t} d t .
\end{gathered}
$$

Therefore

$$
0=\int_{-1}^{1} d t \frac{d^{\ell}\left(t^{2}-1\right)^{\ell}}{d t^{\ell}}\left[\int_{0}^{r} d \rho \rho f(\rho) e^{i \rho t}+r A(r) e^{i r t}\right], \quad l=0,1,2, \ldots
$$

Recall that the Legendre polynomials are defined by the formula

$$
P_{\ell}(t)=\frac{1}{2^{\ell} \ell !} \frac{d^{\ell}}{d t^{\ell}}\left(t^{2}-1\right)^{\ell},
$$

and they form a complete system in $L^{2}(-1,1)$.

Therefore (6.2.11) implies

$$
\int_{0}^{r} d \rho \rho f(\rho) e^{i \rho t}+r A(r) e^{i r t}=0 \quad \forall t \in[-1,1] .
$$

Equation (6.2.13) implies

$$
\int_{0}^{r} d \rho \rho f(\rho) e^{i \rho t}=0, \quad \forall t \in[-1,1]
$$

and

$$
r A(r)=0 .
$$

Therefore $A(r)=0$. Also $f(\rho)=0$ because the left-hand side of (6.2.14) is an entire function of $t$, which vanishes on the interval $[-1,1]$ and, consequently, it vanishes identically, so that $\rho f(\rho)=0$ and therefore $f(\rho) \equiv 0$.

Lemma 6.2 .1 is proved.

We prove that the problem (6.2.7), (6.2.8), (6.2.3), which is a Goursat-type problem, has a solution and this solution is unique in the class of functions $K(r, \rho)$, which are twice continuously differentiable with respect to $\rho$ and $r, \quad 0<r<\infty, \quad 0<\rho \leq r$.

In this section we assume that $q(r) \in C^{1}(0, a)$. This assumption implies that $K(r, \rho)$ is twice continuously differentiable. If (6.1.6) holds, then the arguments in this section which deal with integral equation (6.2.34) remain valid. Specifically, existence and uniqueness of the solution to equation (6.2.34) is proved under the only assumption $\int_{0}^{a} r|q(r)| d r<\infty$ as far as the smoothness of $q(r)$ is concerned.

By a limiting argument one can reduce the smoothness requirements on $q$ to the condition (6.1.6), but in this case equation (6.2.7) has to be understood in distributional sense. 
Let us rewrite the problem we want to study:

$$
\begin{gathered}
r^{2} K_{r r}-\rho^{2} K_{\rho \rho}+\left[r^{2}-r^{2} q(r)-\rho^{2}\right] K(r, \rho)=0, \quad 0<\rho \leq r \\
K(r, r)=\frac{r}{2} \int_{0}^{r} s q(s) d s:=g(r) \\
K(r, 0)=0 .
\end{gathered}
$$

The difficulty in the study of this Goursat-type problem comes from the fact that the coefficients in front of the second derivatives of the kernel $K(r, \rho)$ are variable.

Let us reduce problem (6.2.16) - (6.2.18) to the one with constant coefficients. To do this, introduce the new variables:

$$
\xi=\ln r+\ln \rho, \quad \eta=\ln r-\ln \rho .
$$

Note that

$$
\begin{gathered}
r=e^{\frac{\xi+\eta}{2}}, \quad \rho=e^{\frac{\xi-\eta}{2}}, \\
\eta \geq 0, \quad-\infty<\xi<\infty
\end{gathered}
$$

and

$$
\partial_{r}=\frac{1}{r}\left(\partial_{\xi}+\partial_{\eta}\right), \quad \partial_{\rho}=\frac{1}{\rho}\left(\partial_{\xi}-\partial_{\eta}\right)
$$

Let

$$
K(r, \rho):=B(\xi, \eta) .
$$

A routine calculation transforms equations $(6.2 .16)$ - (6.2.18) to the following ones:

$$
\begin{gathered}
B_{\xi \eta}(\xi, \eta)-\frac{1}{2} B_{\eta}(\xi, \eta)+Q(\xi, \eta) B=0, \quad \eta \geq 0, \quad-\infty<\xi<\infty \\
B(\xi, 0)=g\left(e^{\frac{\xi}{2}}\right):=G(\xi), \quad-\infty<\xi<\infty \\
B(-\infty, \eta)=0, \quad \eta \geq 0
\end{gathered}
$$

where $g(r)$ is defined in $(6.2 .17)$.

Here we have defined

$$
Q(\xi, \eta):=\frac{1}{4}\left[e^{\xi+\eta}-e^{\xi+\eta} q\left(e^{\frac{\xi+\eta}{2}}\right)-e^{\xi-\eta}\right],
$$

and took into account that $\rho=r$ implies $\eta=0$, while $\rho=0$ implies, for any fixed $\eta \geq 0$, that $\xi=-\infty$.

Note that

$$
\begin{gathered}
\sup _{-\infty<\xi<\infty} e^{-\frac{\xi}{2}} G(\xi)<c, \\
\sup _{0 \leq \eta \leq B} \int_{-\infty}^{A}|Q(s, \eta)| d s \leq c(A, B),
\end{gathered}
$$

for any $A \in \mathbb{R}$ and $B>0$, where $c(A, B)>0$ is a constant.

To get rid of the second term on the left-hand side of (6.2.23), let us introduce the new kernel $L(\xi, \eta)$ by the formula:

$$
L(\xi, \eta):=B(\xi, \eta) e^{-\frac{\xi}{2}} .
$$

Then (6.2.23)- (6.2.25) can be written as:

$$
\begin{gathered}
L_{\eta \xi}(\xi, \eta)+Q(\xi, \eta) L(\xi, \eta)=0, \quad \eta \geq 0, \quad-\infty<\xi<\infty, \\
L(\xi, 0)=e^{-\frac{\xi}{2}} G(\xi):=b(\xi):=\frac{1}{2} \int_{0}^{e^{\frac{\xi}{2}}} s q(s) d s, \quad-\infty<\xi<\infty,
\end{gathered}
$$




$$
L(-\infty, \eta)=0, \quad \eta \geq 0 .
$$

We want to prove existence and uniqueness of the solution to (6.2.30) - (6.2.32). In order to choose a convenient Banach space in which to work, let us transform problem (6.2.30) - (6.2.32) to an equivalent Volterra-type integral equation.

Integrate (6.2.30) with respect to $\eta$ from 0 to $\eta$ and use (6.2.31) to get

$$
L_{\xi}(\xi, \eta)-b^{\prime}(\xi)+\int_{0}^{\eta} Q(\xi, t) L(\xi, t) d t=0 .
$$

Integrate (6.2.33) with respect to $\xi$ from $-\infty$ to $\xi$ and use (6.2.33) to get

$$
L(\xi, \eta)=-\int_{-\infty}^{\xi} d s \int_{0}^{\eta} d t Q(s, t) L(s, t)+b(\xi):=V L+b
$$

where

$$
V L:=-\int_{-\infty}^{\xi} d s \int_{0}^{\eta} d t Q(s, t) L(s, t) .
$$

Consider the space $X$ of continuous functions $L(\xi, \eta)$, defined in the half-plane $\eta \geq 0, \quad-\infty<\xi<\infty$, such that for any $B>0$ and any $-\infty<A<\infty$ one has

$$
\|L\|:=\|L\|_{A B}:=\sup _{\substack{-\infty<s \leq A \\ 0 \leq t \leq B}}\left(e^{-\gamma t}|L(s, t)|\right)<\infty
$$

where $\gamma>0$ is a number which will be chosen later so that the operator $V$ in (6.2.34) will be a contraction mapping on the Banach space of functions with norm (6.2.36) for a fixed pair $A, B$. To choose $\gamma>0$, let us estimate the norm of $V$. One has:

$$
\begin{aligned}
\|V L\| \leq \sup _{-\infty<\xi \leq A, 0 \leq \eta \leq B}\left(\int_{-\infty}^{\xi} d s \int_{0}^{\eta} d t|Q(s, t)| e^{-\gamma(\eta-t)} e^{-\gamma t}|L(s, t)|\right) \\
\leq\|L\| \sup _{-\infty<\xi \leq A, 0 \leq \eta \leq B} \int_{-\infty}^{\xi} d s \int_{0}^{\eta} d t\left(2 e^{s+t}+e^{s+t}\left|q\left(e^{\frac{s+t}{2}}\right)\right|\right) e^{-\gamma(\eta-t)} \leq \frac{c}{\gamma}\|L\|,
\end{aligned}
$$

where $c>0$ is a constant depending on $A, B$ and $\int_{0}^{a} r|q(r)| d r$. Indeed, one has:

$$
2 \int_{-\infty}^{A} d s \int_{0}^{\eta} d t e^{s+t-\gamma(\eta-t)}=2 e^{A} \int_{0}^{\eta} d t e^{t-\gamma(\eta-t)} d t \leq 2 e^{A+B} \frac{1-e^{-\gamma B}}{\gamma}=\frac{c_{1}}{\gamma}
$$

and, using the substitution $\sigma=e^{\frac{s+t}{2}}$, one gets:

$$
\begin{gathered}
\int_{-\infty}^{A} d s \int_{0}^{\eta} d t e^{s+t}\left|q\left(e^{\frac{s+t}{2}}\right)\right| e^{-\gamma(\eta-t)}= \\
=\int_{0}^{\eta} d t e^{-\gamma(\eta-t)} \int_{-\infty}^{A} d s e^{s+t}\left|q\left(e^{\frac{s+t}{2}}\right)\right|= \\
=2 \int_{0}^{\eta} d t e^{-\gamma(\eta-t)} \int_{0}^{e^{\frac{A+t}{2}}} d \sigma \sigma|q(\sigma)|= \\
=\frac{2\left(1-e^{-\gamma B}\right)}{\gamma} \int_{0}^{a} d \sigma \sigma|q(\sigma)|:=\frac{c_{2}}{\gamma} .
\end{gathered}
$$

From these estimates inequality (6.2.38) follows. 
It follows from (6.2.38) that $V$ is a contraction mapping in the space $X_{A B}$ of continuous functions in the region $-\infty<\xi \leq A, \quad 0 \leq \eta \leq B$, with the norm (6.2.36) provided that

$$
\gamma>c .
$$

Therefore equation (6.2.34) has a unique solution $L(\xi, \eta)$ in the region

$$
-\infty<\xi<A, \quad 0 \leq \eta \leq B
$$

for any real $A$ and $B>0$ if (6.2.40) holds. This means that the above solution is defined for any $\xi \in \mathbb{R}$ and any $\eta \geq 0$.

Equation (6.2.34) is equivalent to problem (6.2.30) - (6.2.32) and, by (6.2.29), one has:

$$
B(\xi, \eta)=L(\xi, \eta) e^{\frac{\xi}{2}}
$$

Therefore we have proved the existence and uniqueness of $B(\xi, \eta)$, that is, of the kernel $K(r, \rho)=B(\xi, \eta)$ of the transformation operator (6.1.3). Recall that $r$ and $\rho$ are related to $\xi$ and $\eta$ by formulas (6.2.20).

Let us formulate the result:

Theorem 6.2.2. The kernel $K(r, \rho)$ of the transformation operator (6.1.3) solves problem (6.2.16) - (6.2.18). The solution to this problem does exist and is unique in the class of twice continuously differentiable functions for any potential $q(r) \in C^{1}(0, a)$. If $q(r) \in L^{\infty}(0, a)$, then $K(r, \rho)$ has first derivatives which are bounded and equation (6.2.16) has to be understood in the sense of distributions. The following estimate holds for any $r>0$ :

$$
\int_{0}^{r}|K(r, \rho)| \rho^{-1} d \rho<\infty .
$$

Proof of Theorem 6.2.2. We have already proved all the assertions of Theorem 6.2.2 except for the estimate (6.2.43). Let us prove this estimate.

Note that

$$
\int_{0}^{r}|K(r, \rho)| \rho^{-1} d \rho=r \int_{0}^{\infty}|L(2 \ln r-\eta, \eta)| e^{-\frac{\eta}{2}} d \eta<\infty
$$

Indeed, if $r>0$ is fixed, then, by (6.2.20), $\xi+\eta=2 \ln r=$ const. Therefore $d \xi=-d \eta$, and $\rho^{-1} d \rho=$ $\frac{1}{2}(d \xi-d \eta)=-d \eta, \quad \xi=2 \ln r-\eta$. Thus:

$$
\int_{0}^{r}|K(r, \rho)| \rho^{-1} d \rho=\int_{0}^{\infty}|L(2 \ln r-\eta, \eta)| e^{\frac{2 \ln r-\eta}{2}} d \eta=r \int_{0}^{\infty}|L(2 \ln r-\eta, \eta)| e^{-\frac{\eta}{2}} d \eta .
$$

The following estimate holds:

$$
|L(\xi, \eta)| \leq c e^{\left(2+\epsilon_{1}\right)\left[\eta \mu_{1}(\xi+\eta)\right]^{\frac{1}{2}+\epsilon_{2}}},
$$

where $\epsilon_{j}>0, j=1,2$, are arbitrarily small numbers and $\mu_{1}$ is defined in formula (6.2.52) below, see also formula (6.2.49) for the definition of $\mu$.

Estimate (6.2.46) is proved below, in Theorem 6.2.2.

From (6.2.45) and estimate (6.2.56) (see below) estimate (6.2.43) follows. Indeed, denote by $I$ the integral on the right-hand side of (6.2.45). Then, by (6.2.56) one gets:

$$
I \leq 2+2 \sum_{1}^{\infty} \frac{\left[2 \mu_{1}(2 \log r)\right]^{n}}{n !}=2 \exp \left[2 \mu_{1}(2 \log r)\right]<\infty
$$

Theorem 6.2.2 is proved.

Theorem 6.2.3. Estimate (6.2.46) holds. 
Proof of Theorem 6.2.3. From (6.2.34) one gets:

$$
m(\xi, \eta) \leq c_{0}+(W m)(\xi, \eta), \quad m(\xi, \eta):=|L(\xi, \eta)|,
$$

where $c_{0}=\sup _{-\infty<\xi<\infty}|b(\xi)| \leq \frac{1}{2} \int_{0}^{a} s|q(s)| d s$ (see (6.2.31)), and

$$
W m:=\int_{-\infty}^{\xi} d s \int_{0}^{\eta} d t \mu(s+t) m(s, t), \quad \mu(s):=\frac{1}{2} e^{s}\left(1+\left|q\left(e^{\frac{s}{2}}\right)\right|\right) .
$$

It is sufficient to consider inequality (6.2.48) with $c_{0}=1$ : if $c_{0}=1$ and the solution $m_{0}(\xi, \eta)$ to $(6.2 .48)$ satisfies (6.2.46) with $c=c_{1}$, then the solution $m(\xi, \eta)$ of (6.2.48) with any $c_{0}>0$ satisfies (6.2.46) with $c=c_{0} c_{1}$.

Therefore, assume that $c_{0}=1$, then (6.2.48) reduces to:

$$
m(\xi, \eta) \leq 1+(W m)(\xi, \eta) .
$$

Inequality (6.2.46) follows from (6.2.50) by iterations. Let us give the details.

Note that

$$
W 1=\int_{-\infty}^{\xi} d s \int_{0}^{\eta} d t \mu(s+t)=\int_{0}^{\eta} d t \int_{-\infty}^{\xi} d s \mu(s+t)=\int_{0}^{\eta} d t \mu_{1}(\xi+t) \leq \eta \mu_{1}(\xi+\eta) .
$$

Here we have used the notation

$$
\mu_{1}(\xi)=\int_{-\infty}^{\xi} \mu(s) d s
$$

and the fact that $\mu_{1}(s)$ is a monotonically increasing function, since $\mu(s)>0$. Note also that $\mu_{1}(s)<\infty$ for any $s,-\infty<s<\infty$.

Furthermore,

$$
W^{2} 1 \leq \int_{-\infty}^{\xi} d s \int_{0}^{\eta} d t \mu(s+t) t \mu_{1}(s+t) \leq \int_{0}^{\eta} d t t \int_{-\infty}^{\xi} d s \mu(s+t) \mu_{1}(s+t)=\frac{\eta^{2}}{2 !} \frac{\mu_{1}^{2}(\xi+\eta)}{2 !} .
$$

Let us prove by induction that

$$
W^{n} 1 \leq \frac{\eta^{n}}{n !} \frac{\mu_{1}^{n}(\xi+\eta)}{n !} .
$$

For $n=1$ and $n=2$ we have checked (6.2.54). Suppose (6.2.54) holds for some $n$, then

$$
W^{n+1} 1 \leq W\left(\frac{\eta^{n}}{n !} \frac{\mu_{1}^{n}(\xi+\eta)}{n !}\right)=\int_{0}^{\eta} d t \frac{t^{n}}{n !} \int_{-\infty}^{\xi} d s \mu(s+t) \frac{\mu_{1}^{n}(s+t)}{n !} \leq \frac{\eta^{n+1}}{(n+1) !} \frac{\mu_{1}^{n+1}(\xi+\eta)}{(n+1) !} .
$$

By induction, estimate (6.2.53) is proved for all $n=1,2,3, \ldots$. Therefore (6.2.50) implies

$$
m(\xi, \eta) \leq 1+\sum_{n=1}^{\infty} \frac{\eta^{n}}{n !} \frac{\mu_{1}^{n}(\xi+\eta)}{n !} \leq c e^{\left(2+\epsilon_{1}\right)\left[\eta \mu_{1}(\eta+\xi)\right]^{\frac{1}{2}+\epsilon_{2}}},
$$

where we have used Theorem 2 from [Lev, section 1.2], namely the order of the entire function $F(z):=$ $1+\sum_{n=1}^{\infty} \frac{z^{n}}{(n !)^{2}}$ is $\frac{1}{2}$ and its type is 2 . The constant $c>0$ in (6.2.46) depends on $\epsilon_{j}, j=1,2$.

Recall that the order of an entire function $F(z)$ is the number $\rho:=\limsup _{r \rightarrow \infty} \frac{\ln \ln M_{F}(r)}{\ln r}$, where $M_{F}(r):=\max _{|z|=r}|F(z)|$. The type of $F(z)$ is the number $\sigma:=\limsup _{r \rightarrow \infty} \frac{\ln M_{F}(r)}{r^{\rho}}$. It is known [Lev], that if $F(z)=\sum_{n=0}^{\infty} c_{n} z^{n}$ is an entire function, then its order $\rho$ and type $\sigma$ can be calculated by the formulas:

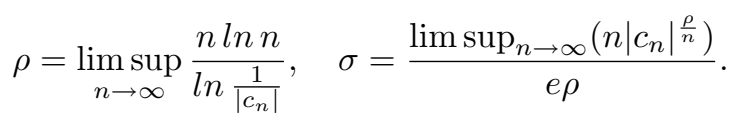

If $c_{n}=\frac{1}{(n !)^{2}}$, then the above formulas yield $\rho=\frac{1}{2}$ and $\sigma=2$. Theorem 6.2.3 is proved. 


\subsection{Uniqueness theorem.}

Denote by $\mathcal{L}$ any fixed subset of the set $\mathbb{N}$ of integers $\{0,1,2, \ldots\}$ with the property:

$$
\sum_{\substack{\ell \in \mathcal{L} \\ \ell \neq 0}} \frac{1}{\ell}=\infty
$$

Theorem 6.3.1. ([R10]) Assume that $q$ satisfies (6.1.6) and (6.3.1) holds. Then the data $\left\{\delta_{\ell}\right\}_{\forall \ell \in \mathcal{L}}$ determine $q$ uniquely.

The idea of the proof is based on property C-type argument.

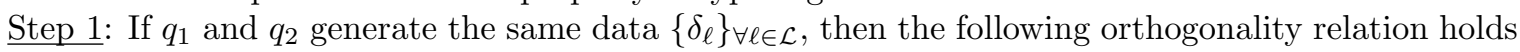
for $p:=q_{1}-q_{2}$ :

$$
h(\ell):=\int_{0}^{a} p(r) \phi_{1 \ell}(r) \phi_{2 \ell}(r) d r=0 \quad \forall \ell \in \mathcal{L},
$$

where $\phi_{j \ell}$ is the scattering solution corresponding to $q_{j}, j=1,2$.

Step 2: Define $h_{1}(\ell):=2^{2 \ell}[\Gamma(\ell+1)]^{2} h(\ell)$, where $\Gamma$ is the Gamma-function. Check that $h_{1}(\ell)$ is holomorphic in $\Pi_{+}:=\{\ell:$ Re $\ell>0\}, \quad \ell=\sigma+i \tau, \quad \sigma \geq 0$, and $\tau$ are real numbers, $h_{1}(\ell) \in N$ (where $N$ is the Nevanlinna class in $\left.\Pi_{+}\right)$, that is

$$
\sup _{0<r<1} \int_{-\pi}^{\pi} \log ^{+}\left|h_{1}\left(\frac{1-r e^{i \varphi}}{1+r e^{i \varphi}}\right)\right| d \varphi<\infty,
$$

where $\log ^{+} x=\left\{\begin{array}{r}\log x \text { if } \log x>0, \\ 0 \text { if } \log x \leq 0 .\end{array}\right.$ If $h_{1} \in N$ vanishes $\forall \ell \in \mathcal{L}$, then $h_{1}=0$ in $\Pi_{+}$, and, by property $C_{\varphi}, p(r)=0$. Theorem 6.3 .1 is proved.

\subsection{Why is the Newton-Sabatier (NS) procedure fundamentally wrong?}

The NS procedure is described in $[\mathrm{N}]$ and $[\mathrm{CS}]$. A vast bibliography of this topic is given in $[\mathrm{CS}]$ and $[\mathrm{N}]$.

Below two cases are discussed. The first case deals with the inverse scattering problem with fixedenergy phase shifts as the data. This problem is understood as follows: an unknown spherically symmetric potential $q$ from an a priori fixed class, say $L_{1,1}$, a standard scattering class, generates fixed-energy phase shifts $\delta_{l}, l=0,1,2, \ldots$, . The inverse scattering problem consists of recovery of $q$ from these data.

The second case deals with a different problem: given some numbers $\delta_{l}, l=0,1,2, \ldots$, , which are assumed to be fixed-energy phase shifts of some potential $q$, from a class not specified, find some potential $q_{1}$, which generates fixed-energy phase shifts equal to $\delta_{l}, l=0,1,2, \ldots$, . This potential $q_{1}$ may have no physical interest because of its non-physical" behavior at infinity or other undesirable properties.

We first discuss NS procedure assuming that it is intended to solve the inverse scattering problem in case 1 . Then we discuss NS procedure assuming that it is intended to solve the problem in case 2 .

\section{Discussion of case 1:}

In $[\mathrm{N} 2]$ and $[\mathrm{N}]$ a procedure was proposed by R. Newton for inverting fixed-energy phase shifts $\delta_{l}, l=0,1,2, \ldots$, corresponding to an unknown spherically symmetric potential $q(r)$. R. Newton did not specify the class of potentials for which he tried to develop an inversion theory and did not formulate and proved any results which would justify the inversion procedure he proposed (NS procedure). His arguments are based on the following claim, which is implicit in his works, but crucial for the validity of NS procedure: 


\section{Claim N1: The basic integral equation.}

$$
K(r, s)=f(r, s)-\int_{0}^{r} K(r, t) f(t, s) \frac{d t}{t^{2}}, \quad 0 \leq s \leq r<\infty,
$$

is uniquely solvable for all $r>0$.

Here

$$
f(r, s):=\sum_{l=0}^{\infty} c_{l} u_{l}(r) u_{l}(s), \quad u_{l}:=\sqrt{\frac{\pi r}{2}} J_{l+\frac{1}{2}}(r),
$$

$c_{l}$ are real numbers, the energy $k^{2}$ is fixed: $k=1$ is taken without loss of generality, $J_{l+\frac{1}{2}}(r)$ are the Bessel functions. If equation (6.4.1) is uniquely solvable for all $r>0$, then the potential $q_{1}$, that NS procedure yields, is defined by the formula:

$$
q_{1}(r)=-\frac{2}{r} \frac{d}{d r} \frac{K(r, r)}{r}
$$

The R. Newton's ansatz (6.4.1) - (6.4.2) for the transformation kernel $K(r, s)$ of the Schroedinger operator, corresponding to some $q(r)$, namely, that $K(r, s)$ is the unique solution to (6.4.1) - (6.4.2), is not correct for a generic potential, as follows from our argument below (see the justification of Conclusions).

If for some $r>0$ equation (6.4.1) is not uniquely solvable, then NS procedure breaks down: it leads to locally non-integrable potentials for which the scattering theory is, in general, not available (see [R9] for a proof of the above statement).

In the original paper [N2] and in his book [N] R. Newton did not study the question, fundamental for any inversion theory: does the reconstructed potential $q_{1}$ generate the data from which it was reconstructed?

In [CS, p. 205], there are two claims:

Claim i) that $q_{1}(r)$ generates the original shifts $\left\{\delta_{l}\right\}$ "provided that $\left\{\delta_{l}\right\}$ are not "exceptional"", and

Claim ii) that NS procedure "yields one (only one) potential which decays faster than $r^{-\frac{3}{2}}$ " and generates the original phase shifts $\left\{\delta_{l}\right\}$.

If one considers NS procedure as a solution to inverse scattering problem of finding an unknown potential $q$ from a certain class, for example $q(r) \in L_{1,1}:=\left\{q: q=\bar{q}, \int_{0}^{\infty} r|q(r)| d r<\infty\right\}$, from the fixed-energy phase shifts, generated by this $q$, then the proof, given in [CS], of Claim i) is not convincing: it is not clear why the potential $q_{1}$, obtained by NS procedure, has the transformation operator generated by the potential corresponding to the original data, that is, to the given fixed-energy phase shifts. In fact, as follows from Proposition 6.4.1 below, the potential $q_{1}$ cannot generate the kernel $K(r, s)$ of the transformation operator corresponding to a generic original potential $q(r) \in L_{1,1}:=\{q$ : $\left.q=\bar{q}, \int_{0}^{\infty} r|q(r)| d r<\infty\right\}$.

Claim ii) is incorrect because the original generic potential $q(r) \in L_{1,1}$ generates the phase shifts $\left\{\delta_{l}\right\}$, and if $q_{1}(r)$, the potential obtained by NS procedure and therefore not equal to $q(r)$ by Proposition 6.4.1 generates the same phase shifts $\left\{\delta_{l}\right\}$, then one has two different potentials $q(r)$ and $q_{1}(r)$, which both decay faster than $r^{-\frac{3}{2}}$ and both generate the original phase shifts $\left\{\delta_{l}\right\}$, contrary to Claim ii).

Our aim is to formulate and justify the following

Conclusions: Claim N1 and ansatz (6.4.1) - (6.4.2) are not proved by R. Newton and, in general, are wrong. Moreover, one cannot approximate with a prescribed accuracy in the norm $\|q\|:=\int_{0}^{\infty} r|q(r)| d r$ a generic potential $q(r) \in L_{1,1}$ by the potentials which might possibly be obtained by the NS procedure. Therefore NS procedure cannot be justified even as an approximate inversion procedure. The NS procedure is fundamentally wrong in the sense that its foundations are wrong.

\section{Let us justify these conclusions:}

Claim N1 formulated above and basic for NS procedure, is wrong, in general, for the following reason: 
Given fixed-energy phase shifts, corresponding to a generic potential $q \in L_{1,1}$, one either cannot carry through NS procedure because:

a) the system (12.2.5a) in [CS], which should determine numbers $c_{l}$ in formula (6.4.2), given the phase shifts $\delta_{l}$, may be not solvable, or

b) if the above system is solvable, equation (6.4.1) may be not (uniquely) solvable for some $r>0$, and in this case NS procedure breaks down since it yields a potential which is not locally integrable (see [R9] for a proof).

If equation (6.4.1) is solvable for all $r>0$ and yields a potential $q_{1}$ by formula (6.4.3), then this potential is not equal to the original generic potential $q \in L_{1,1}$, as follows from Proposition 6.4.1 which is proved in [R9] (see also [ARS]):

Proposition 6.4.1. If equation (6.4.1) is solvable for all $r>0$ and yields a potential $q_{1}$ by formula (6.4.3), then this $q_{1}$ is a restriction to $(0, \infty)$ of a function analytic in a neighborhood of $(0, \infty)$.

Since a generic potential $q \in L_{1,1}$ is not a restriction to $(0, \infty)$ of an analytic function, one concludes that even if equation (6.4.1) is solvable for all $r>0$, the potential $q_{1}$, defined by formula (6.4.3), is not equal to the original generic potential $q \in L_{1,1}$ and therefore the inverse scattering problem of finding an unknown $q \in L_{1,1}$ from its fixed-energy phase shifts is not solved by NS procedure.

The ansatz (6.4.1) - (6.4.2) for the transformation kernel is, in general, incorrect, as follows also from Proposition 6.4.1

Indeed, if the ansatz (6.4.1) - (6.4.2) would be true and formula (6.4.3) would yield the original generic $q$, that is $q_{1}=q$, this would contradict Proposition 6.4.1 If formula (6.4.3) would yield a $q_{1}$ which is different from the original generic $q$, then NS procedure does not solve the inverse scattering problem formulated above. Note also that it is proved in [R10] that independent of the angular momenta $l$ transformation operator, corresponding to a generic $q \in L_{1,1}$ does exist, is unique, and is defined by a kernel $K(r, s)$ which cannot have representation (6.4.2), since it yields by the formula similar to (6.4.3) the original generic potential $q$, which is not a restriction of an analytic in a neighborhood of $(0, \infty)$ function to $(0, \infty)$.

The conclusion, concerning impossibility of approximation of a generic $q \in L_{1,1}$ by potentials $q_{1}$, which can possibly be obtained by NS procedure, is proved in Claim 6.4.3 section 2, see proof of Claim 6.4.3 there.

Thus, our conclusions are justified.

Let us give some additional comments concerning NS procedure.

Uniqueness of the solution to the inverse problem in case 1 was first proved by A. G. Ramm in 1987 (see [R7]) for a class of compactly supported potentials, while R. Newton's procedure was published in [N2], when no uniqueness results for this inverse problem were known. It is still an open problem if for the standard in scattering theory class of $L_{1,1}$ potentials the uniqueness theorem for the solution of the above inverse scattering problem holds.

We discuss the inverse scattering problem with fixed-energy phase shifts (as the data) for potentials $q \in L_{1,1}$, because only for this class of potentials a general theorem of existence and uniqueness of the transformation operators, independent of the angular momenta $l$, has been proved, see [R10]. In $[\mathrm{N} 2],[\mathrm{N}]$, and in $[\mathrm{CS}]$ this result was not formulated and proved, and it was not clear for what class of potentials the transformation operators, independent of $l$, do exist. For slowly decaying potentials the existence of the transformation operators, independent of $l$, is not established, in general, and the potentials, discussed in $[\mathrm{CS}]$ and $[\mathrm{N}]$ in connection with NS procedure, are slowly decaying.

Starting with [N2], [N], and [CS] Claim N1 was not proved or the proofs given (see [CT]) were incorrect (see [R11]). This equation is uniquely solvable for sufficiently small $r>0$, but, in general, it may be not solvable for some $r>0$, and if it is solvable for all $r>0$, then it yields by formula (6.4.3) a potential $q_{1}$, which is not equal to the original generic potential $q \in L_{1,1}$, as follows from Proposition 6.4.1

Existence of "transparent" potentials is often cited in the literature. A "transparent" potential is a potential which is not equal to zero identically, but generates the fixed-energy shifts which are all equal 
to zero.

In [CS, p. 207], there is a remark concerning the existence of "transparent" potentials. This remark is not justified because it is not proved that for the values $c_{l}$, used in [CS, p. 207], equation (6.4.1) is solvable for all $r>0$. If it is not solvable even for one $r>0$, then $N S$ procedure breaks down and the existence of transparent potentials is not established.

In the proof, given for the existence of the "transparent" potentials in [CS, p. 197], formula (12.3.5), is used. This formula involves a certain infinite matrix $M$. It is claimed in [CS, p. 197], that this matrix $M$ has the property $M M=I$, where $I$ is the unit matrix, and on [CS, p. 198], formula (12.3.10), it is claimed that a vector $v \neq 0$ exists such that $M v=0$. However, then $M M v=0$ and at the same time $M M v=v \neq 0$, which is a contradiction. The difficulties come from the claims about infinite matrices, which are not formulated clearly: it is not clear in what space $M$, as an operator, acts, what is the domain of definition of $M$, and on what set of vectors formula (12.3.5) in [CS] holds.

The construction of the "transparent" potential in [CS] is based on the following logic: take all the fixed-energy shifts equal to zero and find the corresponding $c_{l}$ from the infinite linear algebraic system (12.2.7) in [CS]; then construct the kernel $f(r, s)$ by formula (6.4.2) and solve equation (6.4.1) for all $r>0$; finally construct the "transparent" potential by formula (6.4.3). As was noted above, it is not proved that equation (6.4.1) with the constructed above kernel $f(r, s)$ is solvable for all $r>0$. Therefore the existence of the "transparent" potentials is not established.

The physicists have been using NS procedure without questioning its validity for several decades. Apparently the physicists still believe that NS procedure is "an analog of the Gel'fand-Levitan method" for inverse scattering problem with fixed-energy phase shifts as the data. In fact, the NS procedure is not a valid inversion method. Since modifications of NS procedure are still used by some physicists, who believe that this procedure is an inversion theory, the author pointed out some questions concerning this procedure in [ARS] and [R9] and wrote this paper.

This concludes the discussion of case 1 .

\section{Discussion of case 2:}

Suppose now that one wants just to construct a potential $q_{1}$, which generates the phase shifts corresponding to some $q$.

This problem is actually not an inverse scattering problem because one does not recover an original potential from the scattering data, but rather wants to construct some potential which generates these data and may have no physical meaning. Therefore this problem is much less interesting practically than the inverse scattering problem.

However, NS procedure does not solve this problem either: there is no guarantee that this procedure is applicable, that is, that the steps a) and b), described in the justification of the conclusions, can be done, in particular, that equation (6.4.1) is uniquely solvable for all $r>0$.

If these steps can be done, then one needs to check that the potential $q_{1}$, obtained by formula (6.4.3), generates the original phase shifts. This was not done in [N2] and [N].

This concludes the discussion of case 2 .

The rest of the Section contains formulation and proof of Remark 6.4.2 and Claim 6.4.3.

It was mentioned in [N3] that if $Q:=\int_{0}^{\infty} r q(r) d r \neq 0$, then the numbers $c_{l}$ in formula (6.4.2) cannot satisfy the condition $\sum_{0}^{\infty}\left|c_{l}\right|<\infty$. This observation can be obtained also from the following

Remark 6.4.2. For any potential $q(r) \in L_{1,1}$ such that $Q:=\int_{0}^{\infty} r q(r) d r \neq 0$ the basic equation (6.4.1) is not solvable for some $r>0$ and any choice of $c_{l}$ such that $\sum_{l=0}^{\infty}\left|c_{l}\right|<\infty$.

Since generically, for $q \in L_{1,1}$, one has $Q \neq 0$, this gives an additional illustration to the conclusion that equation (6.4.1), in general, is not solvable for some $r>0$. Conditions $\sum_{l=0}^{\infty}\left|c_{l}\right|<\infty$ and $Q \neq 0$ are incompatible.

In [CS, p. 196], a weaker condition $\sum_{l=0}^{\infty} l^{-2}\left|c_{l}\right|<\infty$ is used, but in the examples ([CS, pp. 189-191]), $c_{l}=0$ for all $l \geq l_{0}>0$, so that $\sum_{l=0}^{\infty}\left|c_{l}\right|<\infty$ in all of these examples. 
Claim 6.4.3. The set of the potentials $v(r) \in L_{1,1}$, which can possibly be obtained by the NS procedure, is not dense (in the norm $\|q\|:=\int_{0}^{\infty} r|q(r)| d r$ ) in the set $L_{1,1}$.

Let us prove Remark 6.4.2 and Claim 6.4.3.

Proof of Remark 6.4.2. Writing (6.4.3) as $K(r, r)=-\frac{r}{2} \int_{0}^{r} s q_{1}(s) d s$ and assuming $Q \neq 0$, one gets the following relation:

$$
K(r, r)=-\frac{Q r}{2}[1+o(1)] \rightarrow \infty \text { as } r \rightarrow \infty .
$$

If (6.4.1) is solvable for all $r>0$, then from (6.4.2) and (6.4.1) it follows that $K(r, s)=\sum_{l=0}^{\infty} c_{l} \varphi_{l}(r)$ $u_{l}(s)$, where $\varphi_{l}(r):=u_{l}(r)-\int_{0}^{r} K(r, t) u_{l}(t) \frac{d t}{t^{2}}$, so that $I-K$ is a transformation operator, where $K$ is the operator with kernel $K(r, s), \varphi_{l}^{\prime \prime}+\varphi_{l}-\frac{l(l+1)}{r^{2}} \varphi_{l}-q_{1}(r) \varphi_{l}=0, q_{1}(r)$ is given by (6.4.3), $\varphi_{l}=O\left(r^{l+1}\right)$, as $r \rightarrow 0$,

$$
u_{l}(r) \sim \sin \left(r-\frac{l \pi}{2}\right), \quad \varphi_{l}(r) \sim\left|F_{l}\right| \sin \left(r-\frac{l \pi}{2}+\delta_{l}\right) \text { as } r \rightarrow \infty,
$$

where $\delta_{l}$ are the phase shifts at $k=1$ and $F_{l}$ is the Jost function at $k=1$. One can prove that $\sup _{l}\left|F_{l}\right|<\infty$. Thus, if $\sum_{l=0}^{\infty}\left|c_{l}\right|<\infty$, then

$$
K(r, r)=O(1) \text { as } r \rightarrow \infty .
$$

If $Q \neq 0$ then (6.4.5) contradicts (6.4.4). It follows that if $Q \neq 0$ then equation (6.4.1) cannot be uniquely solvable for all $r>0$, so that NS procedure cannot be carried through if $Q \neq 0$ and $\sum_{l=0}^{\infty}\left|c_{l}\right|<\infty$. This proves Remark 6.4.2.

Proof of Claim 6.4.3. Suppose that $v(r) \in L_{1,1}$ and $Q_{v}:=\int_{0}^{\infty} r v(r) d r=0$, because otherwise NS procedure cannot be carried through as was proved in Remark 6.4.2.

If $Q_{v}=0$, then there is also no guarantee that NS procedure can be carried through. However, we claim that if one assumes that it can be carried through, then the set of potentials, which can possibly be obtained by NS procedure, is not dense in $L_{1,1}$ in the norm $\|q\|:=\int_{0}^{\infty} r|q(r)| d r$. In fact, any potential $q$ such that $Q:=\int_{0}^{\infty} r q(r) d r \neq 0$, and the set of such potentials is dense in $L_{1,1}$, cannot be approximated with a prescribed accuracy by the potentials which can be possibly obtained by the NS procedure.

Let us prove this. Suppose that $q \in L_{1,1}$,

$$
Q_{q}:=\int_{0}^{\infty} r q(r) d r \neq 0, \text { and }\left\|v_{n}-q\right\| \rightarrow 0 \text { as } n \rightarrow \infty,
$$

where the potentials $v_{n} \in L_{1,1}$ are obtained by the NS procedure, so that $Q_{n}:=\int_{0}^{\infty} r v_{n}(r) d r=0$. We assume $v_{n} \in L_{1,1}$ because otherwise $v_{n}$ obviously cannot converge in the norm $\|\cdot\|$ to $q \in L_{1,1}$. Define a linear bounded on $L_{1,1}$ functional

$$
f(q):=\int_{0}^{\infty} r q(r) d r, \quad|f(q)| \leq\|q\|,
$$

where $\|q\|:=\int_{0}^{\infty} r|q(r)| d r$. The potentials $v \in L_{1,1}$, which can possibly be obtained by the NS procedure, belong to the null-space of $f$, that is $f(v)=0$.

If $\lim _{n \rightarrow \infty}\left\|v_{n}-q\right\|=0$, then $\lim _{n \rightarrow \infty}\left|f\left(q-v_{n}\right)\right| \leq \lim _{n \rightarrow \infty}\left\|q-v_{n}\right\|=0$. Since $f$ is a linear bounded functional and $f\left(v_{n}\right)=0$, one gets: $f\left(q-v_{n}\right)=f(q)-f\left(v_{n}\right)=f(q)$. So if $f(q) \neq 0$ then $\lim _{n \rightarrow \infty}\left|f\left(q-v_{n}\right)\right|=|f(q)| \neq 0$. Therefore, no potential $q \in L_{1,1}$ with $Q_{q} \neq 0$ can be approximated arbitrarily accurately by a potential $v(r) \in L_{1,1}$ which can possibly be obtained by the NS procedure. Claim 6.4.3 is proved. 


\subsection{Formula for the radius of the support of the potential in terms of scattering data}

The aim of this Section is to prove formula (6.5.1) for the radius of the support of the potential in terms of the phase shifts. Let us make the following assumption.

Assumption (A): the potential $q(r), r=|x|$, is spherically symmetric, real-valued, $\int_{0}^{a}|q|^{2} d r<\infty$, and $q(r)=0$ for $r>a$, but $q(r) \neq 0$ on $(a-\varepsilon, a)$ for all sufficiently small $\varepsilon>0$.

The number $a>0$ we call the radius of compactness of the potential, or simply the radius of the potential. Let $A\left(\alpha^{\prime}, \alpha\right)$ denote the scattering amplitude corresponding to the potential $q$ at a fixed energy $k^{2}>0$. Without loss of generality let us take $k=1$ in what follows. By $\alpha^{\prime}, \alpha \in S^{2}$ the unit vectors in the direction of the scattered, respectively, incident wave, are meant, $S^{2}$ is the unit sphere in $\mathbb{R}^{3}$. Let us use formulas (5.1.19) and (5.1.20).

It is of interest to obtain some information about $q$ from the (fixed-energy) scattering data, that is, from the scattering amplitude $A\left(\alpha^{\prime}, \alpha\right)$, or, equivalently, from the coefficients $A_{\ell}(\alpha)$. Very few results of such type are known.

A result of such type is a necessary and sufficient condition for $q(x)=q(|x|)$ : it was proved [R, p.131], that $q(x)=q(|x|)$ if and only if $A\left(\alpha^{\prime}, \alpha\right)=A\left(\alpha^{\prime} \cdot \alpha\right)$. Of course, the necessity of this condition was a common knowledge, but the sufficiency, that is, the implication: $A\left(\alpha^{\prime}, \alpha\right)=A\left(\alpha^{\prime} \cdot \alpha\right) \Rightarrow q(x)=q(|x|)$, is a new result $[\mathrm{R} 2]$.

A (modified) conjecture from [R, p.356] says that if the potential $q(x)$ is compactly supported, and $a>0$ is its radius (defined for non-spherically symmetric potentials in the same way as for the spherically symmetric), then

$$
a=\varlimsup_{\ell \rightarrow \infty}\left(\frac{2 \ell}{e}\left[\sup _{\substack{\alpha \in S^{2} \\-\ell \leq m \leq \ell}}\left|A_{\ell m}(\alpha)\right|\right]^{\frac{1}{2 \ell}}\right)=\varlimsup_{\ell \rightarrow \infty}\left(\frac{2 \ell}{e}\left|\delta_{\ell}\right|^{\frac{1}{2 \ell}}\right),
$$

where $\delta_{\ell}$ are the fixed-energy $(k=1)$ phase shifts. We prove (6.5.1) for the spherically symmetric potentials $q=q(r)$.

If $q=q(r)$ then $A_{\ell m}(\alpha)=\widetilde{a}_{\ell} Y_{\ell m}(\alpha)$ where $\widetilde{a}_{\ell}$ depends only on $\ell$ and $k$, but not on $\alpha$ or $\alpha^{\prime}$. Since $k=1$ is fixed, $\tilde{a}_{\ell}$ depends only on $\ell$ for $q=q(r)$. Assuming $q=q(r)$, one takes $A\left(\alpha^{\prime}, \alpha\right)=A\left(\alpha^{\prime} \cdot \alpha\right)$ and calculates $A_{\ell m}(\alpha)=\int_{S^{2}} A\left(\alpha^{\prime} \cdot \alpha\right) \overline{Y_{\ell m}\left(\alpha^{\prime}\right)} d \alpha^{\prime}=\widetilde{a}_{\ell} \overline{Y_{\ell m}(\alpha)}$, where $\widetilde{a}_{\ell}:=\frac{2 \pi}{C_{\ell}^{\left(\frac{1}{2}\right)}(1)} \int_{-1}^{1} A(t) C_{\ell}^{\left(\frac{1}{2}\right)}(t) d t, \quad \ell=$ $0,1,2, \ldots$ Here we have used formula (14.4.46) in [RK, p.413], and $C_{\ell}^{(p)}(t)$ are the Gegenbauer polynomials (see [RK, p.408]). Since $C_{\ell}^{\left(\frac{1}{2}\right)}=P_{\ell}(t), P_{\ell}(1)=1$, where $P_{\ell}(t)$ are the Legendre polynomials (see, e.g., [RK, p.409]), one gets: $\widetilde{a}_{\ell}=2 \pi \int_{-1}^{1} A(t) P_{\ell}(t) d t$.

Formula (6.5.1) for $q=q(r)$ can be written as $a=\varlimsup_{\ell \rightarrow \infty}\left(\frac{2 \ell+1}{e}\left|\widetilde{a}_{\ell}\right|^{\frac{1}{2 \ell}}\right)$.

Indeed, $\sup _{\substack{\alpha \in S^{2} \\-\ell \leq m \leq \ell}}\left|Y_{\ell m}\right|=O\left(\ell^{\frac{1}{2}}\right)$, as is well known (see, e.g., [MP, p.261]). Thus $\varlimsup_{\lim }, \rightarrow \infty$ $\left(\sup _{\substack{\alpha \in S^{2} \\-\ell \leq m \leq \ell}}\left|Y_{\ell m}(\alpha)\right|\right)^{\frac{1}{\ell}}=1$, and formula for (6.5.1) yields:

$$
a=\frac{2}{e} \varlimsup_{\ell \rightarrow \infty}\left(\ell\left|\widetilde{a}_{\ell}\right|^{\frac{1}{2 \ell}}\right) .
$$

Note that assumption (A) implies the following assumption:

Assumption $\left(\mathbf{A}^{\prime}\right)$ : the potential $q(r)$ does not change sign in some left neighborhood of the point a.

This assumption in practice is not restrictive, however, as shown in [R, p.282], the potentials which oscillate infinitely often in a neighborhood of the right end of their support, may have some new properties 
which the potentials without this property do not have. For example, it is proved in [R, p.282], that such infinitely oscillating potentials may have infinitely many purely imaginary resonances, while the potentials which do not change sign in a neighborhood of the right end of their support cannot have infinitely many purely imaginary resonances. Therefore it is of interest to find out if assumption $\mathrm{A}^{\prime}$ is necessary for the validity of (6.5.2).

The main result is:

Theorem 6.5.1. Let assumption (A) hold. Then formula (6.5.2) holds with $\overline{\lim }$ replaced by $\lim$.

This result can be stated equivalently in terms of the fixed-energy phase shift $\delta_{\ell}$ :

$$
\lim _{\ell \rightarrow \infty}\left(\frac{2 \ell+1}{e}\left|\delta_{\ell}\right|^{\frac{1}{2 \ell}}\right)=a .
$$

Below, we prove an auxiliary result:

Lemma 6.5.2. If $q=q(r) \in L^{2}(0, \infty), q(r)$ is real-valued and does not change sign in some interval $\left(a_{1}, a\right]$ where $a_{1}<a$, and $a$ is the radius of $q$, then

$$
a=\lim _{m \rightarrow \infty}\left|\int_{0}^{\infty} q(r) r^{m} d r\right|^{\frac{1}{m}}, m=1,2, \ldots
$$

Below we prove (6.5.3) and, therefore, (6.5.1) for spherically symmetric potentials.

Proof of Lemma 6.5.2. First, we obtain a slightly different result than (6.5.4) as an immediate consequence of the Paley-Wiener theorem. Namely, we prove Lemma 6.5.2 with a continuous parameter $t$ replacing the integer $m$ and $\overline{\lim }$ replacing $\lim$. This is done for $q(r) \in L^{2}(0, a)$ and without additional assumptions about $q$. However, we are not able to prove Lemma 6.5.2 assuming only that $q(r) \in L^{2}(0, a)$.

Since $q(r)$ is compactly supported, one can write

$$
I(t):=\int_{0}^{\infty} q(r) r^{t} d r=\int_{0}^{a} q(r) e^{t \ln r} d r=\int_{-\infty}^{\ln a} q\left(e^{u}\right) e^{u} e^{t u} d u .
$$

Let us recall that Paley-Wiener theorem implies the following claim (see [Lev]):

If $f(z)=\int_{b_{1}}^{b_{2}} g(u) e^{-i u z} d u,\left[b_{1}, b_{2}\right]$ is the smallest interval containing the support of $g(u)$, and $g(u) \in$ $L^{2}\left(b_{1}, b_{2}\right)$, then

$$
b_{2}=\varlimsup_{t \rightarrow+\infty}\left(t^{-1} \ln |f(i t)|\right)=\varlimsup_{t \rightarrow+\infty} \frac{\ln \left|\int_{b_{1}}^{b_{2}} g(u) e^{t u} d u\right|}{t} .
$$

Thus, using (6.5.5) and (6.5.6), one gets:

$$
\ln a=\varlimsup_{t \rightarrow+\infty}\left(t^{-1} \ln \left|\int_{-\infty}^{\ln a} q\left(e^{u}\right) e^{u} e^{t u} d u\right|\right) .
$$

Formula (6.5.7) is similar to (6.5.4) with $m$ replaced by $t$ and lim replaced by $\overline{\lim }$.

Remark 6.5.3. We have used formula (6.5.6) with $b_{1}=-\infty$, while in the Paley-Wiener theorem it is assumed that $b_{1}>-\infty$. However, for $b_{1}<b_{2}, g \not \equiv 0$ on $\left[b_{2}-\varepsilon, b_{2}\right]$ for any $\varepsilon>0$, one has:

$$
\int_{-\infty}^{b_{2}} g(u) e^{t u} d u=\int_{-\infty}^{b_{1}} g(u) e^{t u} d u+\int_{b_{1}}^{b_{2}} g(u) e^{t u} d u:=h_{1}(t)+h_{2}(t) .
$$


Thus $\lim _{t \rightarrow \infty} \frac{h_{1}(t)}{h_{2}(t)}=0$, and

$$
\begin{aligned}
& \varlimsup_{t \rightarrow \infty} \frac{\ln \left|h_{1}(t)+h_{2}(t)\right|}{t} \\
&=\varlimsup_{t \rightarrow \infty} \frac{\ln \left|h_{2}(t)\right|}{t}+\lim _{t \rightarrow \infty} \frac{\ln |1+o(1)|}{t}=\varlimsup_{t \rightarrow \infty} \frac{\ln \left|h_{2}(t)\right|}{t}=\ln a .
\end{aligned}
$$

Therefore formula (6.5.7) follows.

To prove (6.5.4), we use a different approach independent of the Paley-Wiener theorem. We will use (6.5.4) below, in formula (6.5.19). In this formula the role of $q(r)$ in (6.5.4) is played by $r q(r)[1+\epsilon(r, \ell)]$, where $\epsilon=O\left(\frac{1}{\ell}\right)$. Let us prove (6.5.4).

Assume without loss of generality that $q \geq 0$ near $a$. Let $I:=\int_{0}^{a} q(r) r^{m} d r=\int_{0}^{a_{1}} q(r) r^{m} d r+$ $\int_{a_{1}}^{a} q(r) r^{m} d r:=I_{1}+I_{2}$. We have $\left|I_{1}\right|<c a_{1}^{m}, c_{1}(a-\eta)^{m}<I_{2}<c_{2} a^{m}$, where $\eta$ is an arbitrary small positive number. Thus, $I>0$ for all sufficiently large $m$, and $I^{1 / m}=I_{2}^{1 / m}\left(1+\frac{I_{1}}{I_{2}}\right)^{1 / m}$. One has $a-\eta \leq I_{2}^{1 / m} \leq a$ and $\frac{I_{1}}{I_{2}} \rightarrow 0$ as $m \rightarrow \infty$. Since $\eta$ is arbitrary small, it follows that $\lim _{m \rightarrow \infty} I^{1 / m}=a$. This completes the proof of (6.5.4). Lemma 6.5.2 is proved.

Proof of formula (6.5.3). From (5.1.19) and (5.1.23) denoting $a_{\ell}:=e^{i \delta_{\ell}} \sin \delta_{\ell}$, one gets $A\left(\alpha^{\prime} \cdot \alpha\right)=$ $\sum_{\ell=0}^{\infty} \widetilde{a}_{\ell} \overline{Y_{\ell}(\alpha)} Y_{\ell}\left(\alpha^{\prime}\right):=4 \pi \sum_{\ell=0}^{\infty} a_{\ell} \overline{Y_{\ell}(\alpha)} Y_{\ell}\left(\alpha^{\prime}\right)$, where, $a_{\ell}:=\frac{\tilde{a}_{\ell}}{4 \pi}, k=1$, and $a_{\ell}=\frac{e^{2 i \delta_{\ell}}-1}{2 i}=e^{i \delta_{\ell}} \sin \delta_{\ell}$,

$$
a_{\ell}=-\int_{0}^{\infty} d r u_{\ell}(r) q(r) \psi_{\ell}(r),
$$

where $u_{\ell}(r)=r j_{\ell}(r) \sim \sin \left(r-\frac{\ell \pi}{2}\right)$ as $r \rightarrow \infty, j_{\ell}(r)$ are the spherical Bessel functions, $j_{\ell}(r):=$ $\sqrt{\frac{\pi}{2 r}} J_{\ell+\frac{1}{2}}(r)$, and $\psi_{\ell}(r)$ solves (5.1.15) - (5.1.17), and the integral

$$
\psi_{\ell}(r)=u_{\ell}(r)+\int_{0}^{\infty} g_{\ell}(r, s) q(s) \psi_{\ell}(s) d s, \quad k=1,
$$

where

$$
\begin{gathered}
g_{\ell}(r, s)=-u_{\ell}(r) w_{\ell}(s), \quad r<s ; \quad g_{\ell}(r, s)=g_{\ell}(s, r), \\
w_{\ell}(s):=i \sqrt{\frac{\pi s}{2}} H_{\ell+\frac{1}{2}}^{(1)}(s), \quad u_{\ell}(r)=\sqrt{\frac{\pi r}{2}} J_{\ell+\frac{1}{2}}(r),
\end{gathered}
$$

and $H_{\ell}^{(1)}$ is the Hankel function.

It is known [RK, p.407] that

$$
\begin{aligned}
& J_{\nu}(r) \sim\left(\frac{e r}{2 \nu}\right)^{\nu} \frac{1}{\sqrt{2 \pi \nu}}, \quad H_{\nu}^{(1)}(r) \sim-i \sqrt{\frac{2}{\pi \nu}}\left(\frac{e r}{2 \nu}\right)^{-\nu}, \\
& J_{\nu}(r) H_{\nu}^{(1)}(r) \sim-\frac{i}{\pi \nu}, \nu \rightarrow+\infty
\end{aligned}
$$

and [AR, Appendix 4]:

$$
\left|J_{\nu}(r) H_{\nu}^{(1)}(r)\right|<\left(\nu^{2}-\frac{1}{16}\right)^{-\frac{1}{4}}, \quad \nu>\frac{1}{4} .
$$

It follows from (6.5.12) that $u_{\ell}(r)$ does not have zeros on any fixed interval $(0, a]$ if $\ell$ is sufficiently large. Define $v_{\ell}(r):=\frac{\psi_{\ell}(r)}{u_{\ell}(r)}$. Then (6.5.9) yields

$$
v_{\ell}(r)=1+\int_{0}^{a} \frac{g_{\ell}(r, s) u_{\ell}(s)}{u_{\ell}(r)} q(s) v_{\ell}(s) d s .
$$


From (6.5.10) and (6.5.12) one gets

$$
\begin{gathered}
g_{\ell}(r, s) \sim \frac{r}{2 \ell+1}\left(\frac{r}{s}\right)^{\ell}, \quad r<s, \quad \ell \rightarrow+\infty, \\
\frac{u_{\ell}(s)}{u_{\ell}(r)} \sim\left(\frac{s}{r}\right)^{\ell+1}, \quad \ell \rightarrow+\infty .
\end{gathered}
$$

Thus

$$
g_{\ell}(r, s) \frac{u_{\ell}(s)}{u_{\ell}(r)} \sim \frac{s}{2 \ell+1} .
$$

This implies that for sufficiently large $\ell$ equation (6.5.14) has small kernel and therefore is uniquely solvable in $C(0, a)$ and one has

$$
\psi_{\ell}(r)=u_{\ell}(r)\left[1+O\left(\frac{1}{\ell}\right)\right] \text { as } \ell \rightarrow+\infty, \quad 0 \leq r \leq a,
$$

uniformly with respect to $r \in[0, a]$.

In the book [N] formula (12.180), which gives the asymptotic behavior of $S_{\ell}$ for large $\ell$, is misleading: the remainder in this formula is of order which is much greater, in general, than the order of the main term in this formula. That is why we had to find a different approach, which yielded formula (6.5.18).

From (6.5.8), (6.5.11), (6.5.12), and (6.5.18) one has:

$$
\begin{aligned}
a_{\ell} & =-\int_{0}^{\infty} d r q(r) u_{\ell}^{2}(r)\left[1+O\left(\frac{1}{\ell}\right)\right] \\
& =-\int_{0}^{a} d r q(r) r^{2} r^{2 \ell}\left[1+O\left(\frac{1}{\ell}\right)\right] \frac{1}{4 \ell+2}\left(\frac{e}{2 \ell+1}\right)^{2 \ell+1} .
\end{aligned}
$$

Therefore, using (6.5.4), one gets:

$$
\lim _{\ell \rightarrow \infty}\left(\frac{2 \ell+1}{e}\left|a_{\ell}\right|^{\frac{1}{2 \ell}}\right)=\lim _{\ell \rightarrow \infty}\left|\int_{0}^{a} d r q(r) r^{2} r^{2 \ell}\right|^{\frac{1}{2 \ell}}=a .
$$

Theorem 6.5.1 is proved.

Remark 6.5.4. Since $\delta_{\ell} \rightarrow 0$ as $\ell \rightarrow+\infty$, and $\sin \delta_{\ell} \sim \delta_{\ell}$, $e^{i \delta_{\ell}} \sim 1$, as $\delta_{\ell} \rightarrow 0$, formulas (6.5.20) and $a_{\ell}=e^{i \delta_{\ell}} \sin \delta_{\ell}$ imply $\lim _{\ell \rightarrow \infty}\left(\frac{2 \ell+1}{e}\left|\delta_{\ell}\right|^{\frac{1}{2 \ell}}\right)=a$, where $\delta_{\ell}$ is the phase shift at a fixed positive energy. This is formula (6.5.3). 


\section{Chapter 7}

\section{Inverse scattering with "incomplete data"}

\subsection{Uniqueness results}

Consider equation (1.2.3) on the interval $[0,1]$ with boundary conditions $u(0)=u(1)=1$ (or some other selfadjoint homogeneous separated boundary conditions), and $q=\bar{q}, q \in L^{1}[0,1]$. Fix $0<b \leq 1$. Assume $q(x)$ on $[b, 1]$ is known and a subset $\left\{\lambda_{m(n)}\right\}_{\forall n=1,2,3, \ldots}$ of the eigenvalues $\lambda_{n}=k_{n}^{2}$ of the operator $\ell$ corresponding to the chosen boundary conditions is known. Here

$$
\frac{m(n)}{n}=\frac{1}{\sigma}\left(1+\varepsilon_{n}\right), \quad \sigma=\text { const }>0, \quad\left|\varepsilon_{n}\right|<1, \quad \varepsilon_{n} \rightarrow 0 .
$$

We assume sometimes that

$$
\sum_{n=1}^{\infty}\left|\varepsilon_{n}\right|<\infty
$$

Theorem 7.1.1. If (7.1.1) holds and $\sigma>2 b$, then the data $\left\{q(x), b \leq x \leq 1 ;\left\{\lambda_{m(n)}\right\}_{\forall n}\right\}$ determine $q(x)$ on $[0, b]$ uniquely. If (7.1.1) and (7.1.2) hold, the same conclusion holds also if $\sigma=2 b$.

The number $\sigma$ is "the percentage" of the spectrum of $\ell$ which is sufficient to determine $q$ on $[0, b]$ if $\sigma \geq 2 b$ and (7.1.2) holds. For example, if $\sigma=1$ and $b=\frac{1}{2}$, then "one spectrum" determines $q$ on the half-interval $\left[0, \frac{1}{2}\right]$. If $b=\frac{1}{4}, \sigma=\frac{1}{2}$, then "half of the spectrum" determines $q$ on $\left[0, \frac{1}{4}\right]$. Of course, $q$ is assumed known on $[b, 1]$. If $b=1, \sigma=2$, then "two spectra" determines $q$ on the whole interval. By "two spectra" one means the set $\left\{\lambda_{n}\right\} \cup\left\{\mu_{n}\right\}$, where $\left\{\mu_{n}\right\}$ is the set of eigenvalues of $\ell$ corresponding to the same boundary condition $u(0)=0$ at one end, say at $x=0$, and some other selfadjoint boundary condition at the other end, say $u^{\prime}(1)=0$ or $u^{\prime}(1)+h u(1)=0, h=$ const $>0$. The last result is a well-known theorem of Borg, which was strengthened in $[\mathrm{M}]$, where it is proved that not only the potential but the boundary conditions as well are uniquely determined by two spectra. A version of "one spectrum" result was mentioned in [L1, p.81].

Proof of Theorem 7.1.1. First, assume $\sigma>2 b$. If there are $q_{1}$ and $q_{2}$ which produce the same data, then as above, one gets

$$
G(\lambda):=g(k):=\int_{0}^{b} p(x) \varphi_{1}(x, k) \varphi_{2}(x, k) d x=\left.\left(\varphi_{1} w^{\prime}-\varphi_{1}^{\prime} w\right)\right|_{0} ^{b}=\left.\left(\varphi_{1} w^{\prime}-\varphi_{1}^{\prime} w\right)\right|_{x=b}
$$

where $w:=\varphi_{1}-\varphi_{2}, p:=q_{1}-q_{2}, k=\sqrt{\lambda}$. Thus

$$
g(k)=0 \text { at } k= \pm \sqrt{\lambda_{m(n)}}:= \pm k_{n} .
$$


The function $G(\lambda)$ is an entire function of $\lambda$ of order $\frac{1}{2}$ (see (1.2.11) with $k=\sqrt{\lambda}$ ), and is an entire even function of $k$ of exponential type $\leq 2 b$. One has

$$
|g(k)| \leq c \frac{e^{2 b|I m k|}}{1+|k|^{2}}
$$

The indicator of $g$ is defined by the formula

$$
h(\theta):=h_{g}(\theta):=\varlimsup_{r \rightarrow \infty} \frac{\ln \left|g\left(r e^{i \theta}\right)\right|}{r},
$$

where $k=r e^{i \theta}$. Since $|\operatorname{Imk}|=r|\sin \theta|$, one gets from (7.1.5) and (7.1.6) the following estimate

$$
h(\theta) \leq 2 b|\sin \theta| .
$$

It is known [Lev, formula (4.16)] that for any entire function $g(k) \not \equiv 0$ of exponential type one has:

$$
\lim _{r \rightarrow \infty} \frac{n(r)}{r} \leq \frac{1}{2 \pi} \int_{0}^{2 \pi} h_{g}(\theta) d \theta,
$$

where $n(r)$ is the number of zeros of $g(k)$ in the disk $|k| \leq r$. From (7.1.7) one gets

$$
\frac{1}{2 \pi} \int_{0}^{2 \pi} h_{g}(\theta) d \theta \leq \frac{2 b}{2 \pi} \int_{0}^{2 \pi}|\sin \theta| d \theta=\frac{4 b}{\pi}
$$

From (7.1.2) and the known asymptotics of the Dirichlet eigenvalues:

$$
\lambda_{n}=(\pi n)^{2}+c+o(1), \quad n \rightarrow \infty, \quad c=\text { const },
$$

one gets for the number of zeros the estimate

$$
n(r) \geq 2 \sum_{\frac{n \pi}{\sigma}\left[1+0\left(\frac{1}{n^{2}}\right)\right]<r} 1=2 \frac{\sigma r}{\pi}[1+o(1)], \quad r \rightarrow \infty .
$$

From (7.1.8), (7.1.9) and (7.1.11) it follows that

$$
\sigma \leq 2 b
$$

Therefore, if $\sigma>2 b$, then $g(k) \equiv 0$. If $g(k) \equiv 0$ then, by property $C_{\varphi}, p(x)=0$. Theorem 7.1.1 is proved in the case $\sigma>2 b$.

Assume now that $\sigma=2 b$ and

$$
\sum_{n=1}^{\infty}\left|\varepsilon_{n}\right|<\infty
$$

We claim that if an entire function $G(\lambda)$ in (7.1.3) of order $\frac{1}{2}$ vanishes at the points

$$
\lambda_{n}=\frac{n^{2} \pi^{2}}{\sigma^{2}}\left(1+\varepsilon_{n}\right),
$$

and (7.1.13) holds, then $G(\lambda) \equiv 0$. If this is proved, then Theorem 7.1.1 is proved as above.

Let us prove the claim. Define

$$
\Phi(\lambda):=\prod_{n=1}^{\infty}\left(1-\frac{\lambda}{\lambda_{n}}\right)
$$


and recall that

$$
\Phi_{0}(\lambda):=\frac{\sin (\sigma \sqrt{\lambda})}{\sigma \sqrt{\lambda}}=\prod_{n=1}^{\infty}\left(1-\frac{\lambda}{\mu_{n}}\right), \quad \mu_{n}:=\frac{n^{2} \pi^{2}}{\sigma^{2}} .
$$

Since $G\left(\lambda_{n}\right)=0$, the function

$$
w(\lambda):=\frac{G(\lambda)}{\Phi(\lambda)}
$$

is entire, of order $\leq \frac{1}{2}$. Let us use a Phragmen-Lindelöf lemma.

Lemma 7.1.2. [Lev, Theorem 1.22] If an entire function $w(\lambda)$ of order $<1$ has the property sup $_{-\infty<y<\infty}$ $|w(i y)| \leq c$, then $w(\lambda) \equiv c$. If, in addition $w(i y) \rightarrow 0$ as $y \rightarrow+\infty$, then $w(\lambda) \equiv 0$.

We use this lemma to prove that $w(\lambda) \equiv 0$. If this is proved then $G(\lambda) \equiv 0$ and Theorem 7.1.1 is proved.

The function $w(\lambda)$ is entire of order $\frac{1}{2}<1$.

Let us check that

$$
\sup _{-\infty<y<\infty}|w(i y)|<\infty
$$

and that

$$
|w(i y)| \rightarrow 0 \text { as } y \rightarrow+\infty .
$$

One has, using (7.1.5), (7.1.15), (7.1.16) and taking into account that $\sigma=2 b$ :

$$
\begin{aligned}
|w(i y)| & =\left|\frac{G(i y)}{\Phi(i y)} \frac{\Phi_{0}(i y)}{\Phi_{0}(i y)}\right| \leq \frac{e^{2 b|\operatorname{Im} \sqrt{i y}|}}{(1+|y|)}\left(\frac{e^{\sigma|\operatorname{Im} \sqrt{i y}|}}{1+|y|^{\frac{1}{2}}}\right)^{-1}\left(\prod_{h=1}^{\infty} \frac{1+\frac{y^{2}}{\mu_{n}^{2}}}{1+\frac{y^{2}}{\lambda_{n}^{2}}}\right)^{\frac{1}{2}} \\
& \leq \frac{c}{1+|y|^{\frac{1}{2}}}\left(\prod_{\left\{n: \mu_{n} \leq \lambda_{n}\right\}} \frac{\lambda_{n}^{2}}{\mu_{n}^{2}}\right)^{\frac{1}{2}} \leq \frac{c}{1+|y|^{\frac{1}{2}}} \prod_{\left\{n: \mu_{n} \leq \lambda_{n}\right\}}\left(1+\left|\varepsilon_{n}\right|\right) \leq \frac{c_{1}}{1+|y|^{\frac{1}{2}}} .
\end{aligned}
$$

Here we have used elementary inequalities:

$$
\frac{1+a}{1+d} \leq \frac{a}{d} \quad \text { if } \quad a \geq d>0 ; \quad \frac{1+a}{1+d} \leq 1 \quad \text { if } \quad 0 \leq a \leq d,
$$

with $a:=\frac{y^{2}}{\mu_{n}^{2}}, d:=\frac{y^{2}}{\lambda_{n}^{2}}$, and the assumption (7.1.13).

We also used the relation:

$$
\left|\frac{\sin (\sigma \sqrt{i y})}{\sigma \sqrt{i y}}\right| \sim \frac{e^{\sigma|I m \sqrt{i y}|}}{2 \sigma|\sqrt{i y}|} \quad \text { as } \quad y \rightarrow+\infty .
$$

Estimate (7.1.20) implies (7.1.18) and (7.1.19). An estimate similar to (7.1.20) has been used in the literature (see $[\mathrm{GS}]$ ).

Theorem 7.1.1 is proved.

\subsection{Uniqueness results: compactly supported potentials}

Consider the inverse scattering problem of Section 5.2 and assume

$$
q=0 \text { for } x \geq a>0 .
$$

Theorem 7.2.1. If $q \in L_{1,1}$ satisfies (7.2.1), then any one of the data $S(k), \delta(k), f(k), f^{\prime}(k)$, determine q uniquely. 
Proof. We prove first $S(k) \Rightarrow q$. Note that without assumption (7.2.1), or an assumption which implies that $f(k)$ is an entire function on $\mathbb{C}$, the result does not hold. If (7.2.1) holds, or even a weaker assumption:

$$
|q(x)| \leq c_{1} e^{-c_{2}|x|^{\gamma}}, \quad \gamma>1, \quad c_{1}, c_{2}>0,
$$

then $f(k)$, the Jost function (1.2.5), is an entire function of $k$, and $S(k)$ is a meromorphic function on $\mathbb{C}$ with the only poles in $\mathbb{C}_{+}$at the points $i k_{j}, 1 \leq j \leq J$. Thus, $k_{j}$ and $J$ are determined by $S(k)$. Using (1.2.16) and (1.2.11), which holds for all $k \in \mathbb{C}$, because $f(k)$ and $f(x, k)$ are entire functions of $k$, one finds $s_{j}=i \operatorname{Res}_{k=i k_{j}} S(k)$. Thus all the data (1.2.17) are found from $S(k)$ if (7.2.1) (or (7.2.2)) holds. If the data (1.2.17) are known, then $q$ is uniquely determined, see Theorem 5.2.1.

If $\delta(k)$ is given, then $S(k)=e^{2 i \delta(k)}$, so $\delta(k) \Rightarrow q$. If $f(k)$ is given then $S(k)=\frac{f(-k)}{f(k)}$, so $f(k) \Rightarrow q$. If $f^{\prime}(0, k)$ is given, then one can uniquely find $f(k)$ from (1.2.11). Indeed, assume there are two $f(k), f_{1}$ and $f_{2}$, corresponding to the given $f^{\prime}(0, k)$. Subtract from (1.2.11) with $f=f_{1}$ equation (1.2.11) with $f=f_{2}$, denote $f_{1}-f_{2}:=w$, and get $(*) f^{\prime}(0, k) w(-k)=f^{\prime}(0,-k) w(k)$ or $\frac{w(k)}{f^{\prime}(0, k)}=\frac{w(-k)}{f^{\prime}(0,-k)}$. Since $w(\infty)=0$, and $f^{\prime}(0, k)=i k-A(0,0)+\int_{0}^{\infty} A_{x}(0, y) e^{i k y} d y$, one can conclude that $w=0$ if one can check that $\frac{w(k)}{f^{\prime}(0, k)}$ is analytic in $\mathbb{C}_{+}$. The function $f^{\prime}(0, k)$ has at most finitely many zeros in $\mathbb{C}_{+}$, and these zeros are simple. From $(*)$ one concludes that if $f^{\prime}(0, \kappa)=0, \kappa \in \mathbb{C}_{+}$, then $w(\kappa)=0$, because if $f^{\prime}(0, \kappa)=0$ then $f^{\prime}(0,-\kappa) \neq 0$ (see (1.2.11)). Thus $\frac{w(k)}{f^{\prime}(0, k)}$ is analytic in $\mathbb{C}_{+}$. Similarly $\frac{w(-k)}{f^{\prime}(0,-k)}$ is analytic in $\mathbb{C}_{+}$. These two functions agree on the real axis, so, by analytic continuation, the function $\frac{w(k)}{f^{\prime}(0, k)}$ is analytic in $\mathbb{C}_{+}$and vanishes at infinity. Thus it vanishes identically. So $w(k)=0, f_{1}=f_{2}$, and $f(k)$ is uniquely determined by $f^{\prime}(0, k)$. Thus Theorem 7.2 .1 is proved.

\subsection{Inverse scattering on the full line by a potential vanishing on a half-line}

The scattering problem on the full line consists of finding the solution to:

$$
\begin{gathered}
\ell u-k^{2} u=0, \quad x \in \mathbb{R}, \\
u=e^{i k x}+r(k) e^{-i k x}+o(1), \quad x \rightarrow-\infty, \\
u=t(k) e^{i k x}+o(1), \quad x \rightarrow+\infty,
\end{gathered}
$$

where $r(k)$ and $t(k)$ are, respectively, the reflection and transmission coefficients. The above scattering problem describes plane wave scattering by a potential, the plane wave is incident from $-\infty$ in the positive direction of the $x$-axis. The inverse scattering problem consists of finding $q(x)$ given the scattering data

$$
\left\{r(k), k_{j}, s_{j}, 1 \leq j \leq J\right\},
$$

where $s_{j}>0$ are norming constants, $k_{j}>0$, and $-k_{j}^{2}$ are the negative eigenvalues of the operator $\ell_{o}$.

It is known $[\mathrm{M}]$, that the data (7.3.4) determine $q \in L_{1,1}(\mathbb{R}):=\left\{q: q=\bar{q}, \int_{-\infty}^{\infty}(1+|x|)|q| d x<\infty\right\}$ uniquely. Assume that

$$
q(x)=0, \quad x<0 .
$$

Theorem 7.3.1. If $q \in L_{1,1}(\mathbb{R})$ and (7.3.5) holds, then $\{r(k)\}_{\forall k>0}$ determines $q$ uniquely.

Proof. If (7.3.5) holds, then $u=e^{i k x}+r(k) e^{-i k x}$ for $x<0$, and $u=t(k) f(x, k)$ for $x>0$, where $f(k, x)$ is the Jost solution (1.2.5). Thus

$$
\frac{i k(1-r(k))}{1+r(k)}=\frac{u^{\prime}(-0, k)}{u(-0, k)}=\frac{u^{\prime}(+0, k)}{u(+0, k)}=\frac{f^{\prime}(0, k)}{f(k)}:=I(k) .
$$

Therefore $r(k)$ determines $I(k)$, so by Theorem 3.1.2 $q$ is uniquely determined. 


\section{Chapter 8}

\section{Recovery of quarkonium systems}

\subsection{Statement of the inverse problem}

The problem discussed in this Section is: to what extent does the spectrum of a quarkonium system together with other experimental data determines the interquark potential? This problem was discussed in [TQR], where one can find further references. The method given in [TQR] for solving the above problem is this: one has few scattering data $E_{j}, s_{j}$, which will be defined precisely later, and one constructs, using the known results of inverse scattering theory, a Bargmann potential (i.e., a potential which has a rational Jost function) with the same scattering data and considers this a solution to the problem. This approach is wrong because the scattering theory is applicable to the potentials which tend to zero at infinity, while our confining potentials grow to infinity at infinity, and no Bargmann potential can approximate a confining potential on the whole semiaxis $(0, \infty)$. Our aim is to give an algorithm which is consistent and yields a solution to the above problem. The algorithm is based on the Gel'fand-Levitan procedure of Section 4.3.

Let us formulate the problem precisely. Consider the Schroedinger equation

$$
-\nabla^{2} \psi_{j}+q(r) \psi_{j}=E_{j} \psi_{j} \text { in } \mathbb{R}^{3},
$$

where $q(r)$ is a real-valued spherically symmetric potential, $r:=|x|, x \in \mathbb{R}^{3}$,

$$
q(r)=r+p(r), \quad p(r)=o(1) \text { as } r \rightarrow \infty .
$$

The functions $\psi_{j}(x),\left\|\psi_{j}\right\|_{L^{2}\left(\mathbb{R}^{3}\right)}=1$, are the bound states, $E_{j}$ are the energies of these states. We define $u_{j}(r):=r \psi_{j}(r)$, which correspond to $s$-waves, and consider the resulting equation for $u_{j}$ :

$$
\ell u_{j}:=-u_{j}^{\prime \prime}+q(r) u_{j}=E_{j} u_{j}, \quad r>0, \quad u_{j}(0)=0, \quad\left\|u_{j}\right\|_{L^{2}(0, \infty)}=1 .
$$

One can measure the energies $E_{j}$ of the bound states and the quantities $s_{j}=u_{j}^{\prime}(0)$ experimentally.

Therefore the following inverse problem (IP) is of interest:

(IP): given:

$$
\left\{E_{j}, s_{j}\right\}_{\forall j=1,2, \ldots}
$$

can one recover $p(r)$ ?

In [TQR] this question was considered but the approach in [TQR] is inconsistent and no exact results are obtained. The inconsistency of the approach in [TQR] is the following: on the one hand [TQR] uses the inverse scattering theory which is applicable to the potentials decaying sufficiently rapidly at infinity, on the other hand, [TQR] is concerned with potentials which grow to infinity as $r \rightarrow+\infty$. It is nevertheless of some interest that numerical results in [TQR] seem to give some approximation of the potentials in a neighborhood of the origin.

Here we present a rigorous approach to IP and prove the following result: 
Theorem 8.1.1. IP has at most one solution and the potential $q(r)$ can be reconstructed from data (8.1.4) algorithmically.

The reconstruction algorithm is based on the Gel'fand-Levitan procedure for the reconstruction of $q(x)$ from the spectral function. We show that the data (8.1.4) allow one to write the spectral function of the selfadjoint in $L^{2}(0, \infty)$ operator $\ell$, defined by the differential expression (8.1.3) and the boundary condition (8.1.3) at zero.

In Section 8.2 proofs are given and the recovery procedure is described.

Since in experiments one has only finitely many data $\left\{E_{j}, s_{j}\right\}_{1 \leq j \leq J}$, the question arises:

How does one use these data for the recovery of the potential?

We give the following recipe: the unknown confining potential is assumed to be of the form (8.1.2), and it is assumed that for $j>J$ the data $\left\{E_{j}, s_{j}\right\}_{j>J}$ for this potential are the same as for the unperturbed potential $q_{0}(r)=r$. In this case an easy algorithm is given for finding $q(r)$.

This algorithm is described in Section 8.3.

\subsection{Proofs}

We prove Theorem 8.1.1 by reducing (IP) to problem of recovery of $q(r)$ from the spectral function.

Let us recall that the selfadjoint operator $L$ has discrete spectrum since $q(r) \rightarrow+\infty$. The formula for the number of eigenvalues (energies of the bound states), not exceeding $\lambda$, is known:

$$
\sum_{E_{j}<\lambda} 1:=N(\lambda) \sim \frac{1}{\pi} \int_{q(r)<\lambda}[\lambda-q(r)]^{\frac{1}{2}} d r .
$$

This formula yields, under the assumption $q(r) \sim r$ as $r \rightarrow \infty$, the following asymptotics of the eigenvalues:

$$
E_{j} \sim\left(\frac{3 \pi}{2} j\right)^{\frac{2}{3}} \quad \text { as } j \rightarrow+\infty .
$$

The spectral function $\rho(\lambda)$ of the operator $L$ is defined by the formula

$$
\rho(\lambda)=\sum_{E_{j}<\lambda} \frac{1}{\alpha_{j}},
$$

where $\alpha_{j}$ are the normalizing constants:

$$
\alpha_{j}:=\int_{0}^{\infty} \phi_{j}^{2}(r) d r
$$

Here $\phi_{j}(r):=\phi\left(r, E_{j}\right)$ and $\phi(r, E)$ is the unique solution of the problem:

$$
L \phi:=-\phi^{\prime \prime}+q(r) \phi=E \phi, r>0, \phi(0, E)=0, \phi^{\prime}(0, E)=1 .
$$

If $E=E_{j}$, then $\phi_{j}=\phi\left(r, E_{j}\right) \in L^{2}(0, \infty)$. The function $\phi(r, E)$ is the unique solution to the Volterra integral equation:

$$
\phi(r, E)=\frac{\sin (\sqrt{E} r)}{\sqrt{E}}+\int_{0}^{r} \frac{\sin [\sqrt{E}(r-y)]}{\sqrt{E}} q(y) \phi(y, E) d y .
$$

For any fixed $r$ the function $\phi$ is an entire function of $E$ of order $\frac{1}{2}$, that is, $|\phi|<c \exp \left(c|E|^{1 / 2}\right)$, where $c$ denotes various positive constants. At $E=E_{j}$, where $E_{j}$ are the eigenvalues of (8.1.3), one has $\phi\left(r, E_{j}\right):=\phi_{j} \in L^{2}(0, \infty)$. In fact, if $q(r) \sim c r^{a}, a>0$, then $\left|\phi_{j}\right|<c \exp (-\gamma r)$ for some $\gamma>0$.

Let us relate $\alpha_{j}$ and $s_{j}$. From (8.2.3) with $E=E_{j}$ and from (8.1.3), it follows that

$$
\phi_{j}=\frac{u_{j}}{s_{j}} .
$$


Therefore

$$
\alpha_{j}:=\left\|\phi_{j}\right\|_{L^{2}(0, \infty)}^{2}=\frac{1}{s_{j}^{2}} .
$$

Thus data (8.1.4) define uniquely the spectral function of the operator $L$ by the formula:

$$
\rho(\lambda):=\sum_{E_{j}<\lambda} s_{j}^{2} .
$$

Given $\rho(\lambda)$, one can use the Gel'fand-Levitan (GL) method for recovery of $q(r)$. According to this method, define

$$
\sigma(\lambda):=\rho(\lambda)-\rho_{0}(\lambda),
$$

where $\rho_{0}(\lambda)$ is the spectral function of the unperturbed problem, which in our case is the problem with $q(r)=r$, then set

$$
L(x, y):=\int_{-\infty}^{\infty} \phi_{0}(x, \lambda) \phi_{0}(y, \lambda) d \sigma(\lambda)
$$

where $\phi_{0}(x, \lambda)$ are the eigenfunctions of the problem (8.2.3) with $q(r)=r$, and solve the second kind Fredholm integral equation for the kernel $K(x, y)$ :

$$
K(x, y)+\int_{0}^{x} K(x, t) L(t, y) d t=-L(x, y), \quad 0 \leq y \leq x .
$$

The kernel $L(x, y)$ in equation (8.2.10) is given by formula (8.2.9). If $K(x, y)$ solves (8.2.10), then

$$
p(r)=2 \frac{d K(r, r)}{d r}, \quad r>0 .
$$

\subsection{Reconstruction method}

Let us describe the algorithm we propose for recovery of the function $q(x)$ from few experimental data $\left\{E_{j}, s_{j}\right\}_{1 \leq j \leq J}$. Denote by $\left\{E_{j}^{0}, s_{j}^{0}\right\}_{1 \leq j \leq J}$ the data corresponding to $q_{0}:=r$. These data are known and the corresponding eigenfunctions (8.1.3) can be expressed in terms of Airy function Ai(r), which solves the equation $w^{\prime \prime}-r w=0$ and decays at $+\infty$, see [Leb]. The spectral function of the operator $L_{0}$ corresponding to $q=q_{0}:=r$ is

$$
\rho_{0}(\lambda):=\sum_{E_{j}^{0}<\lambda}\left(s_{j}^{0}\right)^{2}
$$

Define

$$
\begin{gathered}
\rho(\lambda):=\rho_{0}(\lambda)+\sigma(\lambda), \\
\sigma(\lambda):=\sum_{E_{j}<\lambda} s_{j}^{2}-\sum_{E_{j}^{0}<\lambda}\left(s_{j}^{0}\right)^{2},
\end{gathered}
$$

and

$$
L(x, y):=\sum_{j=1}^{J} s_{j}^{2} \phi\left(x, E_{j}\right) \phi\left(y, E_{j}\right)-\sum_{j=1}^{J}\left(s_{j}^{0}\right)^{2} \phi_{j}(x) \phi_{j}(y),
$$

where $\phi(x, E)$ can be obtained by solving the Volterra equation (8.2.5) with $q(r)=q_{0}(r):=r$ and represented in the form:

$$
\phi(x, E)=\frac{\sin \left(E^{1 / 2} x\right)}{E^{1 / 2}}+\int_{0}^{x} K(x, y) \frac{\sin \left(E^{1 / 2} y\right)}{E^{1 / 2}} d y,
$$


where $K(x, y)$ is the transformation kernel corresponding to the potential $q(r)=q_{0}(r):=r$, and $\phi_{j}$ are the eigenfunctions of the unperturbed problem:

$$
-\phi_{j}^{\prime \prime}+r \phi_{j}=E_{j} \phi_{j} \quad r>0, \quad \phi_{j}(0)=0, \quad \phi_{j}^{\prime}(0)=1 .
$$

Note that for $E \neq E_{j}^{0}$ the functions (8.3.5) do not belong to $L^{2}(0, \infty)$, but $\phi(0, E)=0$. We denoted in this section the eigenfunctions of the unperturbed problem by $\phi_{j}$ rather than $\phi_{0 j}$ for simplicity of notations, since the eigenfunctions of the perturbed problem are not used in this section. One has: $\phi_{j}(r)=c_{j} A i\left(r-E_{j}^{0}\right)$, where $c_{j}=\left[A i^{\prime}\left(-E_{j}^{0}\right)\right]^{-1}, E_{j}^{0}>0$ is the $j$-th positive root if the equation $A i(-E)=0$ and, by formula (8.2.6), one has $s_{j}^{0}=\left[c_{j}^{2} \int_{0}^{\infty} A i^{2}\left(r-E_{j}^{0}\right) d r\right]^{-1 / 2}$. These formulas make the calculation of $\phi_{j}(x), E_{j}^{0}$ and $s_{j}^{0}$ easy since the tables of Airy functions are available [Leb].

The equation analogous to (8.2.10) is:

$$
K(x, y)+\sum_{j=1}^{2 J} c_{j} \Psi_{j}(y) \int_{0}^{x} K(x, t) \Psi_{j}(t) d t=-\sum_{j=1}^{2 J} c_{j} \Psi_{j}(x) \Psi_{j}(y),
$$

where $\Psi_{j}(t):=\phi\left(t, E_{j}\right), c_{j}=s_{j}^{2}, 1 \leq j \leq J$, and $\Psi_{j}(t)=\phi_{j-J}(t), c_{j}=\left(s_{j-J}^{0}\right)^{2}, J+1 \leq j \leq 2 J$. Equation (8.3.7) has degenerate kernel and therefore can be reduced to a linear algebraic system.

If $K(x, y)$ is found from $(8.3 .7)$, then

$$
p(r)=2 \frac{d}{d r} K(r, r), \quad q(r)=r+p(r) .
$$

Equation (8.2.10) and, in particular (8.3.7), is uniquely solvable by the Fredholm alternative: the homogeneous version of (8.2.10) has only the trivial solution. Indeed, if $h+\int_{0}^{x} L(t, y) h(t) d t=0,0 \leq y \leq x$, then $\|h\|^{2}+\int_{-\infty}^{\infty}|\widetilde{h}|^{2}\left[d \rho(\lambda)-\rho_{0}(\lambda)\right]=0$, so that, by Parseval equality, $\int_{-\infty}^{\infty}|\widetilde{h}|^{2} d \rho(\lambda)=0$. Here $\widetilde{h}:=\int_{0}^{x} h(t) \phi(t, \lambda) d t$, where $\phi(t, \lambda)$ are defined by (8.3.5). This implies that $\widetilde{h}\left(E_{j}\right)=0$ for all $j=1,2, \ldots$ Since $\widetilde{h}(\lambda)$ is an entire function of exponential type $\leq x$, and since the density of the sequence $E_{j}$ is infinite, i.e., $\lim _{\lambda \rightarrow \infty} \frac{N(\lambda)}{\lambda}=\infty$, because $E_{j}=O\left(j^{2 / 3}\right)$, as was shown in the beginning of Section 8.2 , it follows that $\widetilde{h}=0$ and consequently $h(t)=0$, as claimed.

In conclusion consider the case when $E_{j}=E_{j}^{0}, s_{j}=s_{j}^{0}$ for all $j \geq 1$, and $\left\{E_{0}, s_{0}\right\}$ is the new eigenvalue, $E_{0}<E_{1}^{0}$, with the corresponding data $s_{0}$. In this case $L(t, y)=s_{0}^{2} \phi_{0}\left(t, E_{0}\right) \phi_{0}\left(y, E_{0}\right)$, so that equation (8.2.10) takes the form

$$
K(x, y)+s_{0}^{2} \phi_{0}(y) \int_{0}^{x} K(x, t) \phi_{0}\left(t, E_{0}\right) d t=-s_{0}^{2} \phi_{0}\left(x, E_{0}\right) \phi_{0}\left(y, E_{0}\right) .
$$

Thus, one gets:

$$
p(r)=-2 \frac{d}{d r} \frac{s_{0}^{2} \phi_{0}^{2}\left(x, E_{0}\right)}{1+s_{0}^{2} \int_{0}^{x} \phi_{0}^{2}\left(t, E_{0}\right) d t} .
$$




\section{Chapter 9}

\section{Krein's method in inverse scattering}

\subsection{Introduction and description of the method}

Consider inverse scattering problem studied in Chapter 5 and for simplicity assume that there are no bound states. This assumption is removed in Section 9.4.

This chapter is a commentary to Krein's paper [K1]. It contains not only a detailed proof of the results announced in [K1] but also a proof of the new results not mentioned in [K1]. In particular, it contains an analysis of the invertibility of the steps in the inversion procedure based on Krein's results, and a proof of the consistency of this procedure, that is, a proof of the fact that the reconstructed potential generates the scattering data from which it was reconstructed. A numerical scheme for solving inverse scattering problem, based on Krein's inversion method, is proposed, and its advantages compared with the Marchenko and Gel'fand-Levitan methods are discussed. Some of the results are stated in Theorem 9.1.2 - Theorem 9.1.5 below.

Consider the equation for a function $\Gamma_{x}(t, s)$ :

$$
\left(I+H_{x}\right) \Gamma_{x}:=\Gamma_{x}(t, s)+\int_{0}^{x} H(t-u) \Gamma_{x}(u, s) d u=H(t-s), \quad 0 \leq t, s \leq x .
$$

Equation (9.1.1) shows that $\Gamma_{x}=\left(I+H_{x}\right)^{-1} H=I-\left(I+H_{x}\right)^{-1}$, so

$$
\left(I+H_{x}\right)^{-1}=I-\Gamma_{x}
$$

in operator form, and

$$
H_{x}=\left(I-\Gamma_{x}\right)^{-1}-I .
$$

Let us assume that $H(t)$ is a real-valued even function

$$
\begin{gathered}
H(-t)=H(t), \quad H(t) \in L^{1}(\mathbb{R}) \cap L^{2}(\mathbb{R}), \\
1+\widetilde{H}(k)>0, \quad \widetilde{H}(k):=\int_{-\infty}^{\infty} H(t) e^{i k t} d t=2 \int_{0}^{\infty} \cos (k t) H(t) d t .
\end{gathered}
$$

Then (9.1.1) is uniquely solvable for any $x>0$, and there exists a limit

$$
\Gamma(t, s)=\lim _{x \rightarrow \infty} \Gamma_{x}(t, s):=\Gamma_{\infty}(t, s), \quad t, s \geq 0,
$$

where $\Gamma(t, s)$ solves the equation

$$
\Gamma(t, s)+\int_{0}^{\infty} H(t-u) \Gamma(u, s) d u=H(t-s), \quad 0 \leq t, s<\infty .
$$


Given $H(t)$, one solves (9.1.1), finds $\Gamma_{2 x}(s, 0)$, then defines

$$
\psi(x, k):=\frac{E(x, k)-E(x,-k)}{2 i},
$$

where

$$
E(x, k):=e^{i k x}\left[1-\int_{0}^{2 x} \Gamma_{2 x}(s, 0) e^{-i k s} d s\right] .
$$

Formula (9.1.8) gives a one-to-one correspondence between $E(x, k)$ and $\Gamma_{2 x}(s, 0)$.

Remark 9.1.1. In [K1] $\Gamma_{2 x}(0, s)$ is used in place of $\Gamma_{2 x}(s, 0)$ in the definition of $E(x, k)$. By formula (9.2.22) (see Section 9.2 below) one has $\Gamma_{x}(0, x)=\Gamma_{x}(x, 0)$, but $\Gamma_{x}(0, s) \neq \Gamma_{x}(s, 0)$ in general. The theory presented below cannot be constructed with $\Gamma_{2 x}(0, s)$ in place of $\Gamma_{2 x}(s, 0)$ in formula (9.1.8).

Note that

$$
E(x, k)=e^{i k x} f(-k)+o(1), \quad x \rightarrow+\infty,
$$

where

$$
f(k):=1-\int_{0}^{\infty} \Gamma(s) e^{i k s} d s,
$$

and

$$
\Gamma(s):=\lim _{x \rightarrow+\infty} \Gamma_{x}(s, 0):=\Gamma_{\infty}(s, 0) .
$$

Furthermore,

$$
\psi(x, k)=\frac{e^{i k x} f(-k)-e^{-i k x} f(k)}{2 i}+o(1), \quad x \rightarrow+\infty .
$$

Note that $\psi(x, k)=|f(k)| \sin (k x+\delta(k))+o(1), \quad x \rightarrow+\infty$, where $f(k)=|f(k)| e^{-i \delta(k)}, \quad \delta(k)=$ $-\delta(-k), \quad k \in \mathbb{R}$.

The function $\delta(k)$ is called the phase shift. One has $S(k)=e^{2 i \delta(k)}$.

We have changed the notations from [K1] in order to show the physical meaning of the function (9.1.9): $f(k)$ is the Jost function of the scattering theory. The function $\frac{\psi(x, k)}{f(k)}$ is the solution to the scattering problem: it solves equation (1.2.3), and satisfies the correct boundary conditions: $\frac{\psi(0, k)}{f(k)}=0$, and $\frac{\psi(x, k)}{f(k)}=e^{i \delta(k)} \sin (k x+\delta(k))+o(1)$ as $x \rightarrow \infty$.

Krein [K1] calls $S(k):=\frac{f(-k)}{f(k)}$ the $S$-function, and $S(k)$ is the $S$-matrix used in physics.

Assuming no bound states, one can solve the inverse scattering problem (ISP): Given $S(k) \forall k>0$, find $q(x)$.

A solution of the ISP, based on the results of [K1], consists of four steps:

1) Given $S(k)$, find $f(k)$ by solving the Riemann problem (9.2.38).

2) Given $f(k)$, calculate $H(t)$ using the formula

$$
1+\widetilde{H}=1+\int_{-\infty}^{\infty} H(t) e^{i k t} d t=\frac{1}{|f(k)|^{2}} .
$$

3) Given $H(t)$, solve (9.1.6) for $\Gamma_{x}(t, s)$ and then find $\Gamma_{2 x}(2 x, 0), 0 \leq x<\infty$.

4) Define

$$
a(x)=2 \Gamma_{2 x}(2 x, 0),
$$

where

$$
a(0)=2 H(0),
$$

and calculate the potential

$$
q(x)=a^{2}(x)+a^{\prime}(x), \quad a(0)=2 H(0) .
$$


One can also calculate $q(x)$ by the formula:

$$
q(x)=2 \frac{d}{d x}\left[\Gamma_{2 x}(2 x, 0)-\Gamma_{2 x}(0,0)\right] .
$$

Indeed, $2 \Gamma_{2 x}(2 x, 0)=a(x)$, see (9.1.14), $\frac{d}{d x} \Gamma_{2 x}(0,0)=-2 \Gamma_{2 x}(2 x, 0) \Gamma_{2 x}(0,2 x)$, see (9.2.23), and $\Gamma_{2 x}(2 x, 0)$ $=\Gamma_{2 x}(0,2 x)$, see $(9.2 .22)$.

There is an alternative way, based on the Wiener-Levy theorem, to do step 1). Namely, given $S(k)$, find $\delta(k)$, the phase shift, then calculate the function $g(t):=-\frac{2}{\pi} \int_{0}^{\infty} \delta(k) \sin (k t) d k$, and finally calculate $f(k)=\exp \left(\int_{0}^{\infty} g(t) e^{i k t} d k\right)$.

The potential $q \in L_{1,1}$ generates the $S$-matrix $S(k)$, with which we started, provided that the following conditions (9.1.18) - (9.1.21) hold:

$$
S(k)=\overline{S(-k)}=S^{-1}(k), \quad k \in \mathbb{R},
$$

the overbar stands for complex conjugation, and

$$
\begin{gathered}
\operatorname{ind}_{\mathbb{R}} S(k)=0, \\
\|F(x)\|_{L^{\infty}\left(\mathbb{R}_{+}\right)}+\|F(x)\|_{L^{1}\left(\mathbb{R}_{+}\right)}+\left\|x F^{\prime}(x)\right\|_{L^{1}\left(\mathbb{R}_{+}\right)}<\infty,
\end{gathered}
$$

where

$$
F(x):=\frac{1}{2 \pi} \int_{-\infty}^{\infty}[1-S(k)] e^{i k x} d k .
$$

By the index (9.1.19) one means the increment of the argument of $S(k)$ ( when $k$ runs from $-\infty$ to $+\infty$ along the real axis) divided by $2 \pi$. The function (9.1.7) satisfies equation (1.2.5). Recall that we have assumed that there are no bound states.

In Section 9.2 the above method is justified and the following theorems are proved:

Theorem 9.1.2. If (9.1.18) - (9.1.20) hold, then $q(x)$ defined by (9.1.16) is the unique solution to ISP and this $q(x)$ has $S(k)$ as the scattering matrix.

Theorem 9.1.3. The function $f(k)$, defined by (9.1.10), is the Jost function corresponding to potential (9.1.16).

Theorem 9.1.4. Condition (9.1.4) implies that equation (9.1.1) is solvable for all $x \geq 0$ and its solution is unique.

Theorem 9.1.5. If condition (9.1.4) holds, then relation (9.1.11) holds and $\Gamma(s):=\Gamma_{\infty}(s, 0)$ is the unique solution to the equation

$$
\Gamma(s)+\int_{0}^{\infty} H(s-u) \Gamma(u) d u=H(s), \quad s \geq 0 .
$$

The diagram explaining the inversion method for solving ISP, based on Krein's results, can be shown now:

$$
S(k) \underset{s_{1}}{\stackrel{(9.2 .34)}{\Rightarrow}} f(k) \underset{s_{2}}{\stackrel{(9.1 .13)}{\Rightarrow}} H(t) \underset{s_{3}}{\stackrel{(9.1 .1)}{\Rightarrow}} \Gamma_{x}(t, s) \underset{s_{4}}{\stackrel{(\text { trivial })}{\Rightarrow}} \Gamma_{2 x}(2 x, 0) \underset{s_{5}}{\stackrel{(9.1 .14)}{\Rightarrow}} a(x) \underset{s_{6}}{\stackrel{(9.1 .16)}{\Rightarrow}} q(x) .
$$

In this diagram $s_{m}$ denotes step number $m$. Steps $s_{2}, s_{4}, s_{5}$ and $s_{6}$ are trivial. Step $s_{1}$ is almost trivial: it requires solving a Riemann problem with index zero and can be done analytically, in closed form. Step $s_{3}$ is the basic (non-trivial) step which requires solving a family of Fredholm-type linear integral equations (9.1.1). These equations are uniquely solvable if assumption (9.1.4) holds, or if assumptions (9.1.18) - (9.1.20) hold.

In Section 9.2 we analyze the invertibility of the steps in diagram (9.1.23). Note also that, if one assumes (9.1.18) - (9.1.20), diagram (9.1.23) can be used for solving the inverse problems of finding $q(x)$ from the following data: 
a) from $f(k), \forall k>0$,

b) from $|f(k)|^{2}, \forall k>0$, or

c) from the spectral function $d \rho(\lambda)$.

Indeed, if (9.1.18) - (9.1.20) hold, then a) and b) are contained in diagram (9.1.23), and c) follows from the known formula $d \rho(\lambda)=\left\{\begin{array}{rr}\frac{\sqrt{\lambda}}{\pi} \frac{d \lambda}{|f(\sqrt{\lambda})|^{2}}, & \lambda>0, \\ 0, & \lambda<0 .\end{array} \quad\right.$ Let $\lambda=k^{2}$. Then (still assuming (9.1.19)) one has: $d \rho=\frac{2 k^{2}}{\pi} \frac{1}{|f(k)|^{2}} d k, \quad k>0$.

Note that the general case of the inverse scattering problem on the half-axis, when $\operatorname{ind}_{\mathbb{R}} S(k):=\nu \neq 0$, can be reduced to the case $\nu=0$ by the procedure described in Section 9.4, provided that $S(k)$ is the $S$-matrix corresponding to a potential $q \in L_{1,1}\left(\mathbb{R}_{+}\right)$. Necessary and sufficient conditions for this are conditions (9.1.18) - (9.1.20).

Section 9.3 contains a discussion of the numerical aspects of the inversion procedure based on Krein's method. There are advantages in using this procedure (as compared with the Gel'fand-Levitan procedure): integral equation (9.1.1), solving of which constitutes the basic step in the Krein inversion method, is a Fredholm convolution-type equation. Solving such an equation numerically leads to inversion of Toeplitz matrices, which can be done efficiently and with much less computer time than solving the Gel'fand-Levitan equation (1.5.4). Combining Krein's and Marchenko's inversion methods yields an efficient way to solve inverse scattering problems.

Indeed, for small $x$ equation (9.1.1) can be solved by iterations since the norm of the integral operator in (9.1.1) is less than 1 for sufficiently small $x$, say $0<x<x_{0}$. Thus $q(x)$ can be calculated for $0 \leq x \leq \frac{x_{0}}{2}$ by diagram (9.1.23).

For $x \geq x_{0}>0$ one can solve by iterations Marchenko's equation (1.5.13) for the kernel $A(x, y)$, where, if (9.1.19) holds, the function $F(x)$ is defined by the (1.5.11) with $F_{d}=0$.

Indeed, for $x>0$ the norm of the operator in (1.5.11) is less than 1 and it tends to 0 as $x \rightarrow+\infty$.

Finally let us discuss the following question: in the justification of both the Gel'fand-Levitan and Marchenko methods, the eigenfunction expansion theorem and the Parseval relation play a fundamental role. In contrast, the Krein method apparently does not use the eigenfunction expansion theorem and the Parseval relation. However, implicitly, this method is also based on such relations. Namely, assumption (9.1.4) implies that the $S$-matrix corresponding to the potential (9.1.16), has index 0. If, in addition, this potential is in $L_{1,1}\left(\mathbb{R}_{+}\right)$, then conditions (9.1.18) and (9.1.20) are satisfied as well, and the eigenfunction expansion theorem and Parseval's equality hold.

Necessary and sufficient conditions, imposed directly on the function $H(t)$, which guarantee that conditions (9.1.18) - (9.1.20) hold, are not known. However, it follows that conditions (9.1.18) - (9.1.20) hold if and only if $H(t)$ is such that the diagram (9.1.23) leads to a $q(x) \in L_{1,1}\left(\mathbb{R}_{+}\right)$. Alternatively, conditions (9.1.18) - (9.1.20) hold (and consequently, $q(x) \in L_{1,1}\left(\mathbb{R}_{+}\right)$) if and only if condition (9.1.4) holds and the function $f(k)$, which is uniquely defined as the solution to the Riemann problem

$$
\Phi_{+}(k)=[1+\widetilde{H}(k)]^{-1} \Phi_{-}(k), \quad k \in \mathbb{R},
$$

by the formula $f(k)=\Phi_{+}(k)$, generates the $S$-matrix $S(k)$ by formula (9.1.15), and this $S(k)$ satisfies conditions (9.1.18) - (9.1.20). Although the above conditions are verifiable, they are not quite satisfactory because they are implicit, they are not formulated in terms of structural properties of the function $H(t)$ (such as smoothness, rate of decay, etc.).

In Section 9.2 Theorem 9.1.2 - Theorem 9.1.5 are proved. In Section 9.3 numerical aspects of the inversion method based on Krein's results are discussed. In Section 9.4 the ISP with bound states is discussed. In Section 9.5 a relation between Krein's and Gel'fand-Levitan's methods is explained. 


\subsection{Proofs}

Proof of Theorem 9.1.4. If $v \in L^{2}(0, x)$, then

$$
\left(v+H_{x} v, v\right)=\frac{1}{2 \pi}\left[(\widetilde{v}, \widetilde{v})_{L^{2}(\mathbb{R})}+(\widetilde{H} \widetilde{v}, \widetilde{v})_{L^{2}(\mathbb{R})}\right]
$$

where the Parseval equality was used, $\widetilde{v}:=\int_{0}^{x} v(s) e^{i k s} d s$,

$$
(v, v)=\int_{0}^{x}|v|^{2} d s=(v, v)_{L^{2}(\mathbb{R})} .
$$

Thus $I+H_{x}$ is a positive definite selfadjoint operator in the Hilbert space $L^{2}(0, x)$ if (9.1.4) holds. Note that, since $H(t) \in L^{1}(\mathbb{R})$, one has $\widetilde{H}(k) \rightarrow 0$ as $|k| \rightarrow \infty$, so (9.1.4) implies

$$
1+\widetilde{H}(k) \geq c>0 .
$$

A positive definite selfadjoint operator in a Hilbert space is boundedly invertible. Theorem 9.1.4 is proved.

Our argument shows that

$$
\sup _{x \geq 0}\left\|\left(I+H_{x}\right)^{-1}\right\|_{L^{2}(\mathbb{R})} \leq c^{-1} .
$$

Before we prove Theorem 9.1.5, let us prove a simple lemma. For results of this type, see [K2].

Lemma 9.2.1. If (9.1.4) holds, then the operator

$$
H \varphi:=\int_{0}^{\infty} H(t-u) \varphi(u) d u
$$

is a bounded operator in $L^{p}\left(\mathbb{R}_{+}\right), p=1,2, \infty$.

For $\Gamma_{x}(u, s) \in L^{1}\left(\mathbb{R}_{+}\right)$one has

$$
\left\|\int_{x}^{\infty} d u H(t-u) \Gamma_{x}(u, s)\right\|_{L^{2}(0, x)} \leq c_{1} \int_{x}^{\infty} d u\left|\Gamma_{x}(u, s)\right| .
$$

Proof. Let $\|\varphi\|_{p}:=\|\varphi\|_{L^{p}\left(\mathbb{R}_{+}\right)}$. One has

$$
\|H \varphi\|_{1} \leq \sup _{u \in \mathbb{R}_{+}} \int_{0}^{\infty} d t|H(t-u)| \int_{0}^{\infty}|\varphi(u)| d u \leq \int_{-\infty}^{\infty}|H(s)| d s\|\varphi\|_{1}=2\|H\|_{1}\|\varphi\|_{1},
$$

where we have used the assumption $H(t)=H(-t)$. Similarly,

$$
\|H \varphi\|_{\infty} \leq 2\|H\|_{1}\|\varphi\|_{\infty} .
$$

Finally, using Parseval's equality, one gets:

$$
2 \pi\|H \varphi\|_{2}^{2}=\left\|\tilde{H} \tilde{\varphi}_{+}\right\|_{L^{2}(\mathbb{R})}^{2} \leq \sup _{k \in \mathbb{R}}|\widetilde{H}(k)|^{2}\|\varphi\|_{2}^{2}
$$

where

$$
\varphi_{+}(x):=\left\{\begin{array}{rr}
\varphi(x), & x \geq 0 \\
0, & x<0
\end{array}\right.
$$

Since $|\widetilde{H}(k)| \leq 2\|H\|_{1}$ one gets from (9.2.9) the estimate:

$$
\|H \varphi\|_{2} \leq \sqrt{2 / \pi}\|H\|_{1}\|\varphi\|_{2}
$$


To prove (9.2.6), one notes that

$$
\begin{aligned}
\int_{0}^{x} d t\left|\int_{x}^{\infty} d u H(t-u) \Gamma_{x}(u, s)\right|^{2} \leq \sup _{u, v \geq x} \int_{0}^{x} d t|H(t-u) H(t-v)|\left(\int_{x}^{\infty}\left|\Gamma_{x}(u, s)\right| d u\right)^{2} & \\
& \leq c_{1}\left(\int_{x}^{\infty} d u\left|\Gamma_{x}(u, s)\right|\right)^{2} .
\end{aligned}
$$

Estimate (9.2.6) is obtained. Lemma 9.2.1 is proved.

Proof of Theorem 9.1.5. Define $\Gamma_{x}(t, s)=0$ for $t$ or $s$ greater than $x$. Let $w:=\Gamma_{x}(t, s)-\Gamma(t, s)$. Then (9.1.1) and (9.1.6) imply

$$
\left(I+H_{x}\right) w=\int_{x}^{\infty} H(t-u) \Gamma(u, s) d u:=h_{x}(t, s) .
$$

If condition (9.1.4) holds, then equations (9.1.6) and (9.1.22) have solutions in $L^{1}\left(\mathbb{R}_{+}\right)$, and, since $\sup _{t \in \mathbb{R}}|H(t)|<\infty$, it is clear that this solution belongs to $L^{\infty}\left(\mathbb{R}_{+}\right)$and consequently to $L^{2}\left(\mathbb{R}_{+}\right)$, because $\|\varphi\|_{2} \leq\|\varphi\|_{\infty}\|\varphi\|_{1}$. The proof of Theorem 9.1.4 shows that such a solution is unique and does exist. From (9.2.4) one gets

$$
\sup _{x \geq 0}\left\|\left(I+H_{x}\right)^{-1}\right\|_{L^{2}(0, x)} \leq c^{-1}
$$

For any fixed $s>0$ one sees that $\sup _{x \geq y}\left\|h_{x}(t, s)\right\| \rightarrow 0$ as $y \rightarrow \infty$, where the norm here stands for any of the three norms $L^{p}(0, x), p=1,2, \infty$. Therefore (9.2.12) and (9.2.11) imply

$$
\begin{aligned}
\|w\|_{L^{2}(0, x)}^{2} & \leq c^{-2}\left\|h_{x}\right\|_{L^{2}(0, x)}^{2} \\
& \leq c^{-2}\left\|\int_{x}^{\infty} H(t-u) \Gamma(u, s) d u\right\|_{L^{1}(0, x)}\left\|\int_{x}^{\infty} H(t-u) \Gamma(u, s) d u\right\|_{L^{\infty}(0, x)} \\
& \leq \text { const }\|\Gamma(u, s)\|_{L^{1}(x, \infty)}^{2} \rightarrow 0 \text { as } x \rightarrow \infty
\end{aligned}
$$

since $\Gamma(u, s) \in L^{1}\left(\mathbb{R}_{+}\right)$for any fixed $s>0$ and $H(t) \in L^{1}(\mathbb{R})$.

Also

$$
\begin{gathered}
\|w(t, s)\|_{L^{\infty}(0, x)}^{2} \leq 2\left(\left\|h_{x}\right\|_{L^{\infty}(0, x)}^{2}+\left\|H_{x} w\right\|_{L^{\infty}(0, x)}^{2}\right) \\
\leq c_{1}\|\Gamma(u, s)\|_{L^{1}(x, \infty)}^{2}+c_{2} \sup _{t \in \mathbb{R}}\|H(t-u)\|_{L^{2}(0, x)}^{2}\|w\|_{L^{2}(0, x)}^{2},
\end{gathered}
$$

where $c_{j}>0$ are some constants. Finally, by (9.2.6), one has;

$$
\|w(t, s)\|_{L^{2}(0, x)}^{2} \leq c_{3}\left(\int_{x}^{\infty}|\Gamma(u, s)| d u\right)^{2} \rightarrow 0 \text { as } x \rightarrow+\infty .
$$

From (9.2.15) and (9.2.17) relation (9.1.11) follows. Theorem 9.1.5 is proved.

Let us now prove Theorem 9.1.3. We need several lemmas.

Lemma 9.2.2. The function (9.1.8) satisfies the equations

$$
\begin{gathered}
E^{\prime}=i k E-a(x) E_{-}, \quad E(0, k)=1, \quad E_{-}:=E(x,-k), \\
E_{-}^{\prime}=-i k E_{-}-a(x) E, \quad E_{-}(0, k)=1,
\end{gathered}
$$

where $E^{\prime}=\frac{d E}{d x}$, and $a(x)$ is defined in (9.1.14). 
Proof. Differentiate (9.1.8) and get

$$
E^{\prime}=i k E-e^{i k x}\left(2 \Gamma_{2 x}(2 x, 0) e^{-i k 2 x}+2 \int_{0}^{2 x} \frac{\partial \Gamma_{2 x}(s, 0)}{\partial(2 x)} e^{-i k s} d s\right) .
$$

We will check below that

$$
\frac{\partial \Gamma_{x}(t, s)}{\partial x}=-\Gamma_{x}(t, x) \Gamma_{x}(x, s)
$$

and

$$
\Gamma_{x}(t, s)=\Gamma_{x}(x-t, x-s) .
$$

Thus, by (9.2.21),

$$
\frac{\partial \Gamma_{2 x}(s, 0)}{\partial(2 x)}=-\Gamma_{2 x}(s, 2 x) \Gamma_{2 x}(2 x, 0) .
$$

Therefore (9.2.20) can be written as

$$
E^{\prime}=i k E-e^{-i k x} a(x)+a(x) e^{i k x} \int_{0}^{2 x} \Gamma_{2 x}(s, 2 x) e^{-i k s} d s .
$$

By (9.2.22) one gets

$$
\Gamma_{2 x}(s, 2 x)=\Gamma_{2 x}(2 x-s, 0) .
$$

Thus

$$
\begin{aligned}
e^{i k x} \int_{0}^{2 x} \Gamma_{2 x}(s, 2 x) e^{-i k s} d s & =\int_{0}^{2 x} \Gamma_{2 x}(2 x-s, 0) e^{i k(x-s)} d s \\
& =e^{-i k x} \int_{0}^{2 x} \Gamma_{2 x}(y, 0) e^{i k y} d y .
\end{aligned}
$$

From (9.2.24) and (9.2.26) one gets (9.2.18).

Equation (9.2.19) can be obtained from $(9.2 .18)$ by changing $k$ to $-k$. Lemma 9.2.2 is proved if formulas (9.2.21) - (9.2.22) are checked.

To check (9.2.22), use $H(-t)=H(t)$ and compare the equation for $\Gamma_{x}(x-t, x-s):=\varphi$,

$$
\Gamma_{x}(x-t, x-s)+\int_{0}^{x} H(x-t-u) \Gamma_{x}(u, x-s) d u=H(x-t-x+s)=H(t-s),
$$

with equation (9.1.1). Let $u=x-y$. Then (9.2.27) can be written as

$$
\varphi+\int_{0}^{x} H(t-y) \varphi d y=H(t-s)
$$

which is equation (9.1.1) for $\varphi$. Since (9.1.1) has at most one solution, as we have proved above (Theorem 9.1.4), formula $(9.2 .22)$ is proved.

To prove (9.2.21), differentiate (9.1.1) with respect to $x$ and get:

$$
\Gamma_{x}^{\prime}(t, s)+\int_{0}^{x} H(t-u) \Gamma_{x}^{\prime}(u, s) d u=-H(t-x) \Gamma_{x}(x, s), \quad \Gamma_{x}^{\prime}:=\frac{\partial \Gamma_{x}}{\partial x} .
$$

Set $s=x$ in (9.1.1), multiply (9.1.1) by $-\Gamma_{x}(x, s)$, compare with (9.2.29) and use again the uniqueness of the solution to (9.1.1). This yields (9.2.21).

Lemma 9.2.2 is proved.

Lemma 9.2.3. Equation (1.2.5) holds for $\psi$ defined in (9.1.7). 
Proof. From (9.1.7) and (9.2.18) - (9.2.19) one gets

$$
\psi^{\prime \prime}=\frac{E^{\prime \prime}-E_{-}^{\prime \prime}}{2 i}=\frac{\left(i k E-a(x) E_{-}\right)^{\prime}-\left(-i k E_{-}-a(x) E\right)^{\prime}}{2 i} .
$$

Using (9.2.18) - (9.2.19) again one gets

$$
\psi^{\prime \prime}=-k^{2} \psi+q(x) \psi, \quad q(x):=a^{2}(x)+a^{\prime}(x) .
$$

Lemma 9.2 .3 is proved.

Proof of Theorem 9.1.3. The function $\psi$ defined in (9.1.7) solves equation (1.2.5) and satisfies the conditions

$$
\psi(0, k)=0, \quad \psi^{\prime}(0, k)=k .
$$

The first condition is obvious (in [K1] there is a misprint: it is written that $\psi(0, k)=1$ ), and the second condition follows from (9.1.7) and (9.2.19):

$$
\psi^{\prime}(0, k)=\frac{E^{\prime}(0, k)-E_{-}^{\prime}(0, k)}{2 i}=\left.\frac{i k E-a E_{-}-\left(i k E_{-}-a E\right)}{2 i}\right|_{x=0}=\frac{2 i k}{2 i}=k .
$$

Let $f(x, k)$ be the Jost solution. Since $f(x, k)$ and $f(x,-k)$ are linearly independent, one has $\psi=$ $c_{1} f(x, k)+c_{2} f(x,-k)$, where $c_{1}, c_{2}$ are some constants independent of $x$ but depending on $k$.

From (9.2.32) one gets $c_{1}=\frac{f(-k)}{2 i}, \quad c_{2}=\frac{-f(k)}{2 i} ; \quad f(k):=f(0, k)$. Indeed, the choice of $c_{1}$ and $c_{2}$ guarantees that the first condition (9.2.32) is obviously satisfied, while the second follows from the Wronskian formula: $f^{\prime}(0, k) f(-k)-f(k) f^{\prime}(0,-k)=2 i k$.

Comparing this with (9.1.12) yields the conclusion of Theorem 9.1.3.

\section{Invertibility of the steps of the inversion procedure and proof of Theorem 1.1}

Let us start with a discussion of the inversion steps 1) - 4) described in the introduction.

Then we discuss the uniqueness of the solution to ISP and the consistency of the inversion method, that is, the fact that $q(x)$, reconstructed from $S(k)$ by steps 1$)-4)$, generates the original $S(k)$.

Let us go through steps 1) - 4) of the reconstruction method and prove their invertibility. The consistency of the inversion method follows from the invertibility of the steps of the inversion method.

Step 1. $S(k) \Rightarrow f(k)$.

Assume $S(k)$ satisfying (9.1.18) - (9.1.20) is given. Then solve the Riemann problem

$$
f(k)=S(-k) f(-k), \quad k \in \mathbb{R} .
$$

Since $\operatorname{ind}_{\mathbb{R}} S(k)=0$, one has $\operatorname{ind}_{\mathbb{R}} S(-k)=0$. Therefore the problem (9.2.33) of finding an analytic function $f_{+}(k)$ in $\mathbb{C}_{+}:=\{k: \operatorname{Im} k>0\}, f(k):=f_{+}(k)$ in $\mathbb{C}_{+}$, (and an analytic function $f_{-}(k):=f(-k)$ in $\left.\mathbb{C}_{-}:=\{k: \operatorname{Im} k<0\},\right)$ from equation $(9.2 .33)$ can be solved in closed form. Namely, define

$$
f(k)=\exp \left\{\frac{1}{2 \pi i} \int_{-\infty}^{\infty} \frac{\ln S(-y) d y}{y-k}\right\}, \quad \operatorname{Im} k>0 .
$$

Then $f(k)$ solves (9.2.33), $f_{+}(k)=f(k), f_{-}(k)=f(-k)$. Indeed,

$$
\ln f_{+}(k)-\ln f_{-}(k)=\ln S(-k), \quad k \in \mathbb{R}
$$


by the known jump formula for the Cauchy integral. Integral (9.2.34) converges absolutely at infinity, $\ln S(-y)$ is differentiable with respect to $y$ for $y \neq 0$, and is bounded on the real axis, so the Cauchy integral in (9.2.34) is well defined.

To justify the above claims, one uses the known properties of the Jost function

$$
f(k)=1+\int_{0}^{\infty} A(0, y) e^{i k y} d y:=1+\int_{0}^{\infty} A(y) e^{i k y} d y,
$$

where estimates (1.2.26) and (1.2.27) hold and $A(y)$ is a real-valued function. Thus

$$
\begin{gathered}
f(k)=1-\frac{A(0)}{i k}-\frac{1}{i k} \int_{0}^{\infty} A^{\prime}(t) e^{i k t} d t \\
S(-k)=\frac{f(k)}{f(-k)}=\frac{1-\frac{A(0)}{i k}-\frac{1}{i k} \widetilde{A^{\prime}}(k)}{1+\frac{A(0)}{i k}+\frac{1}{i k} \widetilde{A^{\prime}}(-k)}=1+O\left(\frac{1}{k}\right) .
\end{gathered}
$$

Therefore

$$
\ln S(-k)=O\left(\frac{1}{k}\right) \quad \text { as } \quad|k| \rightarrow \infty, \quad k \in \mathbb{R} .
$$

Also

$$
\dot{f}(k)=i \int_{0}^{\infty} A(y) y e^{i k y} d y, \quad \dot{f}:=\frac{\partial f}{\partial k} .
$$

Estimate (1.2.26) implies

$$
\int_{0}^{\infty} y|A(y)| d y \leq 2 \int_{0}^{\infty} t|q(t)| d t<\infty, \quad A(y) \in L^{2}\left(\mathbb{R}_{+}\right),
$$

so that $\dot{f}(k)$ is bounded for all $k \in \mathbb{R}, f(k)-1 \in L^{2}(\mathbb{R}), S(-k)$ is differentiable for $k \neq 0$, and $\ln S(-y)$ is bounded on the real axis, as claimed. Note that

$$
f(-k)=\overline{f(k)}, \quad k \in \mathbb{R} .
$$

The converse step $f(k) \Rightarrow S(k)$ is trivial: $S(k)=\frac{f(-k)}{f(k)}$. If $\operatorname{ind}_{\mathbb{R}} S=0$ then $f(k)$ is analytic in $\mathbb{C}_{+}$, $f(k) \neq 0$ in $\mathbb{C}_{+}, f(k)=1+O\left(\frac{1}{k}\right)$ as $|k| \rightarrow \infty, k \in \mathbb{C}_{+}$, and (9.2.42) holds.

Step 2. $\quad f(k) \Rightarrow H(t)$.

This step is done by formula (9.1.13):

$$
H(t)=\frac{1}{2 \pi} \int_{-\infty}^{\infty} e^{-i k t}\left(\frac{1}{|f(k)|^{2}}-1\right) d k
$$

One has $H \in L^{2}(\mathbb{R})$. Indeed, it follows from (9.2.37) that

$$
|f(k)|^{2}-1=-\frac{2}{k} \int_{0}^{\infty} A^{\prime}(t) \sin (k t) d t+O\left(\frac{1}{|k|^{2}}\right), \quad|k| \rightarrow \infty, \quad k \in \mathbb{R} .
$$

The function

$$
w(k):=\frac{1}{k} \int_{0}^{\infty} A^{\prime}(t) \sin (k t) d t
$$

is continuous because $A^{\prime}(t) \in L^{1}\left(\mathbb{R}_{+}\right)$by $(1.2 .27)$, and $w \in L^{2}(\mathbb{R})$ since $w=o\left(\frac{1}{|k|}\right)$ as $|k| \rightarrow \infty, k \in \mathbb{R}$. Thus, $H \in L^{2}(\mathbb{R})$.

Also, $H \in L^{1}(\mathbb{R})$. Indeed, integrating by parts, one gets from (9.2.43) the relation: $2 \pi H(t)=$ $\frac{i}{t} \int_{-\infty}^{\infty} e^{-i k t}[\dot{f}(k) f(-k)-\dot{f}(-k) f(k)] \frac{d k}{|f(k)|^{4}}:=\frac{i}{t} g(t)$, and $g \in L^{2}(\mathbb{R})$, therefore $H \in L^{1}(\mathbb{R})$. To check that 
$g \in L^{2}(\mathbb{R})$, one uses $(9.2 .36),(1.2 .26)-(1.2 .27)$, and $(9.2 .40)-(9.2 .41)$, to conclude that $[\dot{f}(k) f(-k)-$ $\dot{f}(-k) f(k)] \in L^{2}(\mathbb{R})$, and, since $f(k) \neq 0$ on $\mathbb{R}$ and $f(\infty)=1$, it follows that $g \in L^{2}(\mathbb{R})$. The inclusion $[\dot{f}(k) f(-k)-\dot{f}(-k) f(k)] \in L^{2}(\mathbb{R})$ follows from (9.2.36), (1.2.26) - (1.2.27), and (9.2.40) - (9.2.41).

By (9.2.43), the function $H^{\prime}(t)$ is the Fourier transform of $-i k\left(1-|f(k)|^{2}\right)|f(k)|^{-2}$, and, by (9.2.44), $k\left(|f(k)|^{2}-1\right)=-2 \int_{0}^{\infty} A^{\prime}(t) \sin (k t) d t+O\left(\frac{1}{|k|}\right)$, as $|k| \rightarrow \infty, \quad k \in \mathbb{R}$. Thus, $H^{\prime}(t)$ behaves, essentially, as $A^{\prime}(t)$ plus a function, whose Fourier transform is $O\left(\frac{1}{|k|}\right)$. Estimate (1.2.27) shows how $A^{\prime}(t)$ behaves. Equation (9.1.1) shows that $\Gamma_{x}(t, 0)$ is as smooth as $H(t)$, so that formula (9.1.17) for $q(x)$ shows that $q$ is essentially as smooth as $A^{\prime}(t)$.

The converse step

$$
H(t) \Rightarrow f(k)
$$

is also done by formula (9.1.13): Fourier inversion gives $|f(k)|^{2}=f(k) f(-k)$, and factorization of $|f(k)|^{2}$ yields the unique $f(k)$, since $f(k)$ does not vanish in $\mathbb{C}_{+}$and tends to 1 at infinity.

Step 3. $H \Rightarrow \Gamma_{x}(s, 0) \Rightarrow \Gamma_{2 x}(2 x, 0)$.

This step is done by solving equation (9.1.1). By Theorem 9.1.4 equation (9.1.1) is uniquely solvable since condition (9.1.4) is assumed. Formula (9.1.13) holds and the known properties of the Jost function are used: $f(k) \rightarrow 1$ as $k \rightarrow \pm \infty, f(k) \neq 0$ for $k \neq 0, k \in \mathbb{R}, f(0) \neq 0$ since $\operatorname{ind}_{\mathbb{R}} S(k)=0$.

The converse step $\Gamma_{x}(s, 0) \Rightarrow H(t)$ is done by formula (9.1.3). The converse step

$$
\Gamma_{2 x}(2 x, 0) \Rightarrow \Gamma_{x}(s, 0)
$$

constitutes the essence of the inversion method.

This step is done as follows:

$$
\Gamma_{2 x}(2 x, 0) \stackrel{(9.1 .14)}{\Rightarrow} a(x) \stackrel{(9.2 .18)--(9.2 .19)}{\Rightarrow} E(x, k) \stackrel{(9.1 .8)}{\Rightarrow} \Gamma_{x}(s, 0) .
$$

Given $a(x)$, system (9.2.18) - (9.2.19) is uniquely solvable for $E(x, k)$.

Note that the step $q(x) \Rightarrow f(k)$ can be done by solving the uniquely solvable integral equation (1.2.6): with $q \in L_{1,1}\left(\mathbb{R}_{+}\right)$, and then calculating $f(k)=f(0, k)$.

Step 4. $\quad a(x):=2 \Gamma_{2 x}(2 x, 0) \Rightarrow q(x)$.

This step is done by formula (9.1.16). The converse step

$$
q(x) \Rightarrow a(x)
$$

can be done by solving the Riccati problem (9.1.16) for $a(x)$ given $q(x)$ and the initial condition $2 H(0)$. Given $q(x)$, one can find $2 H(0)$ as follows: one finds $f(x, k)$ by solving equation (1.2.6), which is uniquely solvable if $q \in L_{1,1}\left(\mathbb{R}_{+}\right)$, then one gets $f(k):=f(0, k)$, and then calculates $2 H(0)$ using formula (9.2.43) with $t=0$ :

$$
2 H(0)=\frac{1}{\pi} \int_{-\infty}^{\infty}\left(\frac{1}{|f(k)|^{2}}-1\right) d k .
$$

Proof of Theorem 9.1.2. If (9.1.18) - (9.1.20) hold, then, as has been proved in Section 5.5, there is a unique $q(x) \in L_{1,1}\left(\mathbb{R}_{+}\right)$which generates the given $S$-matrix $S(k)$.

It is not proved in [K1] that $q(x)$ defined in (1.19) (and obtained as a final result of steps 1) - 4)) generates the scattering matrix $S(k)$ with which we started the inversion.

Let us now prove this. We have already discussed the following diagram:

$$
S(k) \stackrel{(9.2 .34)}{\Leftrightarrow} f(k) \stackrel{(9.1 .13)}{\Leftrightarrow} H(t) \stackrel{(9.1 .1)}{\Leftrightarrow} \Gamma_{x}(s, 0) \Rightarrow \Gamma_{2 x}(2 x, 0) \stackrel{(9.1 .14)}{\Leftrightarrow} a(x) \stackrel{(9.1 .16)}{\Leftrightarrow} q(x) .
$$

To close this diagram and therefore establish the basic one-to-one correspondence $S(k) \Leftrightarrow q(x)$, one needs to prove $\Gamma_{2 x}(2 x, 0) \Rightarrow \Gamma_{x}(s, 0)$. This is done by the scheme (9.2.48). 
Note that the step $q(x) \Rightarrow a(x)$ requires solving Riccati equation (9.1.16) with the boundary condition $a(0)=2 H(0)$. Existence of the solution to this problem on all of $\mathbb{R}_{+}$is guaranteed by the assumptions (9.1.18) - (9.1.20). The fact that these assumptions imply $q(x) \in L_{1,1}\left(\mathbb{R}_{+}\right)$is proved in Section 5.5. Theorem 9.1.2 is proved.

Uniqueness theorems for the inverse scattering problem are not given in [K1]. They can be found in Section 5.5

Remark 9.2.4. From our analysis one gets the following result:

Proposition 9.2.5. If $q(x) \in L_{1,1}\left(\mathbb{R}_{+}\right)$and has no bounds states and no resonance at zero, then Riccati equation (9.1.16) with the initial condition (9.1.15) has the solution a $(x)$ defined for all $x \in \mathbb{R}_{+}$.

\subsection{Numerical aspects of the Krein inversion procedure.}

The main step in this procedure from the numerical viewpoint is to solve equation (9.1.1) for all $x>0$ and all $0<s<x$, which are the parameters in equation (9.1.1).

Since equation (9.1.1) is an equation with the convolution kernel, its numerical solution involves inversion of a Toeplitz matrix, which is a well developed area of numerical analysis. Moreover, such an inversion requires much less computer memory and time than the inversion based on the Gel'fand-Levitan or Marchenko methods. This is the main advantage of Krein's inversion method.

This method may become even more attractive if it is combined with the Marchenko method. In the Marchenko method the equation to be solved is (1.5.13) where $F(x)$ is defined in (1.5.11) and is known if $S(k)$ is known. The kernel $A(x, y)$ is to be found from (1.5.11) and if $A(x, y)$ is found then the potential is recovered by the formula: Equation (1.5.11) can be written in operator form: $\left(I+F_{x}\right) A=-F$. The operator $F_{x}$ is a contraction mapping in the Banach space $L^{1}(x, \infty)$ for $x>0$. The operator $H_{x}$ in (9.1.1) is a contraction mapping in $L^{\infty}(0, x)$ for $0<x<x_{0}$, where $x_{0}$ is chosen to that $\int_{0}^{x_{0}}|H(t-u)| d u<1$. Therefore it seems reasonable from the numerical point of view to use the following approach:

1. Given $S(k)$, calculate $f(k)$ and $H(t)$ as explained in Steps 1 and 2, and also $F(x)$ by formula (1.5.11).

2. Solve by iterations equation (9.1.1) for $0<x<x_{0}$, where $x_{0}$ is chosen so that the iteration method for solving (9.1.6) converges rapidly. Then find $q(x)$ as explained in Step 4.

3. Solve equation (1.5.13) for $x>x_{0}$ by iterations. Find $q(x)$ for $x>x_{0}$ by formula (1.5.12).

\subsection{Discussion of the ISP when the bound states are present.}

If the given data are (9.1.15), then one defines $w(k)=\prod_{j=1}^{J} \frac{k-i k_{j}}{k+i k_{j}} \quad$ if $\quad \operatorname{ind}_{\mathbb{R}} S(x)=-2 J$ and $W(k)=$ $\frac{k}{k+i \gamma} w(k)$ if $\operatorname{ind}_{R} S(k)=-2 J-1$, where $\gamma>0$ is arbitrary, and is chosen so that $\gamma \neq k_{j}, 1 \leq j \leq J$. Then one defines $S_{1}(k):=S(k) w^{2}(k)$ if $\operatorname{ind}_{\mathbb{R}} S=-2 J$ or $S_{1}(k):=S(k) W^{2}(k)$ if $\operatorname{ind}_{\mathbb{R}} S=$ $-2 J-1$. Since $\operatorname{ind}_{\mathbb{R}} w^{2}(k)=2 J$ and $\operatorname{ind}_{\mathbb{R}} W^{2}(k)=2 J+1$, one has $\operatorname{ind}_{\mathbb{R}} S_{1}(k)=0$. The theory of Section 9.2 applies to $S_{1}(k)$ and yields $q_{1}(x)$. From $q_{1}(x)$ one gets $q(x)$ by adding bound states $-k_{j}^{2}$ and norming constants $s_{j}$ using the known procedure (e.g. see $[\mathrm{M}]$ ).

\subsection{Relation between Krein's and GL's methods.}

The GL (Gel'fand-Levitan) method in the case of absence of bound states of the following steps (see Chapter 4, for example): 
Step 1. Given $f(k)$, the Jost function, find

$$
\begin{aligned}
L(x, y) & :=\frac{2}{\pi} \int_{0}^{\infty} d k k^{2}\left(\frac{1}{|f(k)|^{2}}-1\right) \frac{\sin k x}{k} \frac{\sin k y}{k} \\
& =\frac{1}{\pi} \int_{0}^{\infty} d k\left(|f(k)|^{-2}-1\right)(\cos [k(x-y)]-\cos [k(x+y)]) \\
& :=M(x-y)-M(x+y),
\end{aligned}
$$

where $M(x):=\frac{1}{\pi} \int_{0}^{\infty} d k\left(|f(k)|^{-2}-1\right) \cos (k x)$.

Step 2. Solve the integral equation (1.5.4) for $K(x, y)$.

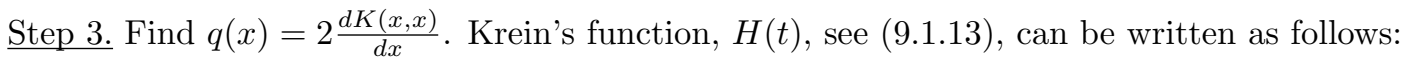

$$
H(t)=\frac{1}{2 \pi} \int_{-\infty}^{\infty}\left(|f(k)|^{-2}-1\right) e^{-i k t} d k=\frac{1}{\pi} \int_{0}^{\infty}\left(|f(k)|^{-2}-1\right) \cos (k t) d k .
$$

Thus, the relation between the two methods is given by the formula:

$$
M(x)=H(x) .
$$

In fact, the GL method deals with the inversion of the spectral foundation $d \rho$ of the operator $-\frac{d^{2}}{d x^{2}}+$ $q(x)$ defined in $L^{2}\left(\mathbb{R}_{+}\right)$by the Dirichlet boundary condition at $x=0$. However, if $\operatorname{ind}_{\mathbb{R}} S(k) \stackrel{d x^{2}}{=} 0$ (in this case there are no bound states and no resonance at $k=0$ ), then (see (1.2.21)): $d \rho(\lambda)=$ $\left\{\begin{aligned} \frac{2 k^{2} d k}{\pi|f(k)|^{2}}, & \lambda>0, \quad \lambda=k^{2}, \quad \text { so } d \rho(\lambda) \text { in this case is uniquely defined by } f(k), k \geq 0 . \\ 0, & \lambda<0,\end{aligned}\right.$ 


\section{Chapter 10}

\section{Inverse problems for the heat and wave equations.}

\subsection{Inverse problem for the heat equation}

Consider problem (1.5.25) - (1.5.28). Assume

$$
a(t)=0 \text { for } t>T, \quad \int_{0}^{T} a(t) d t<\infty, \quad a(t) \not \equiv 0 .
$$

One can also take $a(t)=\delta(t)$ where $\delta(t)$ is the delta-function. We prove that the inverse problem of finding $q(x) \in L^{1}[0,1], q=\bar{q}$, from the conditions (1.5.25) - (1.5.28) has at most one solution.

If (1.5.28) is replaced by the condition

$$
u_{x}(0, t)=b_{0}(t)
$$

then $q(x)$, in general, is not uniquely defined by the conditions (1.5.25), (1.5.26), (1.5.27) and (10.1.2), but $q$ is uniquely defined by these data if, for example, $q\left(\frac{1}{2}-x\right)=q\left(\frac{1}{2}+x\right)$, or if $q(x)$ is known on $\left[\frac{1}{2}, 1\right]$.

Let us take the Laplace transform of $(1.5 .25)-(1.5 .28)$ and put $v(x, \lambda):=\int_{0}^{\infty} u(x, t) e^{-\lambda t} d t, A(\lambda):=$ $v(1, \lambda), B(\lambda):=v_{x}(1, \lambda), B_{0}(\lambda):=v_{x}(0, \lambda)$. Then (1.5.25) - (1.5.28) can be written as

$$
\begin{gathered}
\ell v+\lambda v:=-v^{\prime \prime}+q(x) v+\lambda v=0, \quad 0 \leq x \leq 1, \quad v(0, \lambda)=0, \quad v(1, \lambda)=A(\lambda) \\
v^{\prime}(1, \lambda)=B(\lambda)
\end{gathered}
$$

and (10.1.2) takes the form

$$
v^{\prime}(0, \lambda)=B_{0}(\lambda)
$$

Theorem 10.1.1. The data $\{A(\lambda), B(\lambda)\}$, known on a set of $\lambda \in(0, \infty)$, which has a finite positive limit point, determine $q$ uniquely.

Proof. Since $A(\lambda)$ and $B(\lambda)$ are analytic in $\prod_{+}:=\{\lambda: \Re \lambda>0\}$, one can assume that $A(\lambda)$ and $B(\lambda)$ are known for all $\lambda>0$. If $k=i \lambda^{\frac{1}{2}}$ and $\varphi$ is defined in (1.2.3) then $v(x, \lambda)=c(k) \varphi(x, k), c(k) \neq 0$, $A(\lambda)=c(k) \varphi(1, k), B(\lambda)=c(k) \varphi^{\prime}(1, k)$, so

$$
\frac{B(\lambda)}{A(\lambda)}=\frac{\varphi^{\prime}(1, k)}{\varphi(1, k)}
$$

Thus the function $\frac{B(\lambda)}{A(\lambda)}$ is meromorphic in $\mathbb{C}$, its zeros on the axis $k \geq 0$ are the eigenvalues of $\ell=$ $-\frac{d^{2}}{d x^{2}}+q(x)$, corresponding to the boundary conditions $u(0)=u^{\prime}(1)=0$ and its poles on the axis $k \geq 0$ 
are the eigenvalues of $\ell$ corresponding to $u(0)=u(1)=0$. The knowledge of two spectra determines $q$ uniquely (Section 7.1).

An alternative proof of Theorem 10.1.1, based on property $C_{\varphi}$, is: assume that $q_{1}$ and $q_{2}$ generate the same data, $p:=q_{1}-q_{2}, w:=v_{1}-v_{2}$, where $v_{j}, j=1,2$, solves $(10.1 .3)-(10.1 .4)$ with $q=q_{j}$, and get $(*) \ell_{1} w=p v_{2}, w(0, \lambda)=w(1, \lambda)=w^{\prime}(1, \lambda)=0$. Multiply $(*)$ by $\varphi_{1}, \ell_{1} \varphi_{1}+\lambda \varphi_{1}=0, \varphi_{1}(0, \lambda)=0$, $\varphi^{\prime}\left({ }_{1}(0, \lambda)=1\right.$, and integrate over $[0,1]$ to get

$$
\int_{0}^{1} p v_{2} \varphi_{1} d x=0 \quad \forall \lambda>0
$$

By property $C_{\varphi}$ it follows from (10.1.7) that $p=0$. Theorem 10.1.1 is proved.

Theorem 10.1.2. Data (10.1.3), (10.1.5) do not determine $q$ uniquely in general. They do if $q(x)$ is known on $\left[\frac{1}{2}, 1\right]$, or if $q\left(x+\frac{1}{2}\right)=q\left(\frac{1}{2}-x\right)$.

Proof. Arguing as in the first proof of Theorem 10.1.1, one concluded that the data (10.1.3), (10.1.5) yields only one (Dirichlet) spectrum of $\ell$, since $\varphi^{\prime}(0, k)=1$. One spectrum determines $q$ only on "a half of the interval", $b=\frac{1}{2}$, see Section 7.1. Theorem 10.1.2 is proved.

\subsection{What are the "correct" measurements?}

From Theorem 10.1.1 and Theorem 10.1.2 it follows that the measurements $\left\{u_{x}(1, t)\right\}_{\forall t>0}$ are much more informative than $\left\{u_{x}(0, t)\right\}_{\forall t>0}$ for the problem (1.5.25) - (1.5.27). In this section we state a similar result for the problem

$$
\begin{gathered}
u_{t}=\left(a(x) u^{\prime}\right)^{\prime}, \quad 0 \leq x \leq 1, \quad t>0 ; \quad u(x, 0)=0, \quad u(0, t)=0, \\
u(1, t)=f(t) .
\end{gathered}
$$

The extra data, that is, measurements, are

$$
a(1) u^{\prime}(1, t)=g(t)
$$

which is the flux. Assume:

$$
f \not \equiv 0, \quad f \in L^{1}(0,1), \quad a(x) \in W^{2,1}(0,1), \quad a(x) \geq c>0,
$$

$W^{\ell, p}$ is the Sobolev space. Physically, $a(x)$ is the conductivity, $u$ is the temperature. We also consider in place of (10.2.3) the following data:

$$
a(0) u^{\prime}(0, t)=h(t) .
$$

Our results are similar to those in Section 10.1:

data $\{f(t), g(t)\}_{\forall t>0}$ determine $q(x)$ uniquely, while data $\{f(t), h(t)\}_{\forall t>0}$ do not, in general, determine a $(x)$ uniquely.

Therefore, the measurements $\{g(t)\}_{\forall t>0}$ are much more informative than the measurements $\{h(t)\}_{\forall t>0}$. We refer the reader to [R9].

\subsection{Inverse problem for the wave equation}

Consider inverse problem (1.5.20) - (1.5.24). Our result is

Theorem 10.3.1. The above inverse problem has at most one solution. 
Proof. Take the Fourier transform of (1.5.20) - (1.5.24) and get:

$$
\begin{gathered}
\ell v-k^{2} v=0, \quad x \geq 0, \quad v(x, k)=\int_{0}^{\infty} e^{i k t} u(x, t) d t \\
v(0, k)=1, \quad v(1, k)=A(k)=\int_{0}^{\infty} a(t) e^{i k t} d t .
\end{gathered}
$$

From (10.3.1) one gets $v(x, k)=c(k) f(x, k)$, where $f(x, k)$ is the Jost solution, and from (10.3.2) one gets $v(x, k)=\frac{f(x, k)}{f(k)}$ and $A(k)=\frac{f(1, k)}{f(k)}=\frac{e^{i k}}{f(k)}$, because $q=0$ for $x>1$. Thus $f(k)=\frac{e^{i k}}{A(k)}$ is known. By Theorem 7.2.1, $q$ is uniquely determined. Theorem 10.3 .1 is proved.

Remark 10.3.2. The above method allows one to consider other boundary conditions at $x=0$, such as $u^{\prime}(0, t)=0$ or $u^{\prime}(0, t)=h u(0, t), h=$ const $>0$, and different data at $x=1$, for example, $u^{\prime}(1, t)=b(t)$. 


\section{Chapter 11}

\section{Inverse problem for an inhomogeneous Schrödinger equation}

In thisSecyion an inverse problem is studied for an inhomogeneous Schrödinger equation. Most of the earlier studies dealt with inverse problems for homogeneous equations. Let

$$
\ell u-k^{2} u:=-u^{\prime \prime}+q(x) u-k^{2} u=\delta(x), \quad x \in \mathbb{R}^{1}, \quad \frac{\partial u}{\partial|x|}-i k u \rightarrow 0, \quad|x| \rightarrow \infty .
$$

Assume that $q(x)$ is a real-valued function, $q(x)=0$ for $|x|>1, \quad q \in L^{\infty}[-1,1]$. Suppose that the data $\{u(-1, k), u(1, k)\}, \quad \forall k>0$ are given.

The inverse problem is:

(IP) Given the data, find $q(x)$.

This problem is of practical interest: think about finding the properties of an inhomogeneous slab (the governing equation is plasma equation) from the boundary measurements of the field, generated by a point source inside the slab. Assume that the self-adjoint operator $\ell=-\frac{d^{2}}{d x^{2}}+q(x)$ in $L^{2}(\mathbb{R})$ has no negative eigenvalues (this is the case when $q(x) \geq 0$, for example). The operator $\ell$ is the closure in $L^{2}(\mathbb{R})$ of the symmetric operator $\ell_{0}$ defined on $C_{0}^{\infty}\left(\mathbb{R}^{1}\right)$ by the formula $\ell_{0} u=-u^{\prime \prime}+q(x) u$. Our result is:

Theorem 11.1.3. Under the above assumptions IP has at most one solution.

Proof of Theorem 11.1: The solution to (11.1.1) is

$$
u= \begin{cases}\frac{g(k)}{[f, g]} f(x, k), & x>0, \\ \frac{f(k)}{[f, g]} g(x, k), & x<0 .\end{cases}
$$

Here $f(x, k)$ and $g(x, k)$ solve homogeneous version of equation (11.1.1) and have the following asymptotics:

$$
\begin{gathered}
f(x, k) \sim e^{i k x}, \quad x \rightarrow+\infty, \quad g(x, k) \sim e^{-i k x}, \quad x \rightarrow-\infty, \\
f(k):=f(0, k), \quad g(k):=g(0, k), \\
{[f, g]:=f g^{\prime}-f^{\prime} g=-2 i k a(k),}
\end{gathered}
$$

where the prime denotes differentiation with respect to $x$-variable, and $a(k)$ is defined by the equation

$$
f(x, k)=b(k) g(x, k)+a(k) g(x,-k) .
$$


It is known that

$$
\begin{gathered}
g(x, k)=-b(-k) f(x, k)+a(k) f(x,-k), \\
a(-k)=\overline{a(k)}, \quad b(-k)=\overline{b(k)}, \quad|a(k)|^{2}=1+|b(k)|^{2}, \quad k \in \mathbb{R}, \\
a(k)=1+O\left(\frac{1}{k}\right), \quad k \rightarrow \infty, \quad k \in \mathbb{C}_{+} ; \quad b(k)=O\left(\frac{1}{k}\right), \quad|k| \rightarrow \infty, \quad k \in \mathbb{R}, \\
{[f(x, k), g(x,-k)]=2 i k b(k), \quad[f(x, k), g(x, k)]=-2 i k a(k),}
\end{gathered}
$$

$a(k)$ in analytic in $\mathbb{C}_{+}, b(k)$ in general does not admit analytic continuation from $\mathbb{R}$, but if $q(x)$ is compactly supported, then $a(k)$ and $b(k)$ are analytic functions of $k \in \mathbb{C} \backslash 0$.

The functions

$$
A_{1}(k):=\frac{g(k) f(1, k)}{-2 i k a(k)}, \quad A_{2}(k):=\frac{f(k) g(-1, k)}{-2 i k a(k)}
$$

are the data, they are known for all $k>0$. Therefore one can assume the functions

$$
h_{1}(k):=\frac{g(k)}{a(k)}, \quad h_{2}(k):=\frac{f(k)}{a(k)}
$$

to be known for all $k>0$ because

$$
f(1, k)=e^{i k}, \quad g(-1, k)=e^{i k},
$$

as follows from the assumption $q=0$ if $|x|>1$, and from (11.1.3).

From (11.1.12), (11.1.7) and (11.1.6) it follows that

$$
\begin{gathered}
a(k) h_{1}(k)=-b(-k) f(k)+a(k) f(-k)=-b(-k) h_{2}(k) a(k)+h_{2}(-k) a(-k) a(k), \\
a(k) h_{2}(k)=b(k) a(k) h_{1}(k)+a(k) h_{1}(-k) a(-k) .
\end{gathered}
$$

From (11.1.14) and (11.1.15) it follows:

$$
\begin{gathered}
-b(-k) h_{2}(k)+h_{2}(-k) a(-k)=h_{1}(k), \\
b(k) h_{1}(k)+a(-k) h_{1}(-k)=h_{2}(k) .
\end{gathered}
$$

Eliminating $b(-k)$ from (11.1.16) and (11.1.17), one gets:

$$
a(k) h_{1}(k) h_{2}(k)+a(-k) h_{1}(-k) h_{2}(-k)=h_{1}(k) h_{1}(-k)+h_{2}(-k) h_{2}(k),
$$

or

$$
a(k)=m(k) a(-k)+n(k), \quad k \in \mathbb{R}
$$

where

$$
m(k):=-\frac{h_{1}(-k) h_{2}(-k)}{h_{1}(k) h_{2}(k)}, \quad n(k):=\frac{h_{1}(-k)}{h_{2}(k)}+\frac{h_{2}(-k)}{h_{1}(k)} .
$$

Problem (11.1.19) is a Riemann problem for the pair $\{a(k), a(-k)\}$, the function $a(k)$ is analytic in $\mathbb{C}_{+}:=\{k: k \in \mathbb{C}, \operatorname{Im} k>0\}$ and $a(-k)$ is analytic in $\mathbb{C}_{-}$. The functions $a(k)$ and $a(-k)$ tend to one as $k$ tends to infinity in $\mathbb{C}_{+}$and, respectively, in $\mathbb{C}_{-}$, see equation (11.1.9).

The function $a(k)$ has finitely many simple zeros at the points $i k_{j}, 1 \leq j \leq J, k_{j}>0$, where $-k_{j}^{2}$ are the negative eigenvalues of the operator $\ell$ defined by the differential expression $\ell u=-u^{\prime \prime}+q(x) u$ in $L^{2}(\mathbb{R})$.

The zeros $i k_{j}$ are the only zeros of $a(k)$ in the upper half-plane $k$.

Define

$$
\operatorname{ind} a(k):=\frac{1}{2 \pi i} \int_{-\infty}^{\infty} d \ln a(k) .
$$


One has

$$
\text { ind } a=J,
$$

where $J$ is the number of negative eigenvalues of the operator $\ell$, and, using (11.1.12), (11.1.22) and (11.1.20), one gets

$$
\operatorname{ind} m(k)=-2\left[\operatorname{ind} h_{1}(k)+\operatorname{ind} h_{2}(k)\right]=-2[\operatorname{ind} g(k)+\operatorname{ind} f(k)-2 J] .
$$

Since $\ell$ has no negative eigenvalues, it follows that $J=0$.

In this case ind $f(k)=$ ind $g(k)=0$ (see Lemma 1 below), so ind $m(k)=0$, and $a(k)$ is uniquely recovered from the data as the solution of (11.1.19) which tends to one at infinity, see equation (11.1.9). If $a(k)$ is found, then $b(k)$ is uniquely determined by equation (11.1.17) and so the reflection coefficient $r(k):=\frac{b(k)}{a(k)}$ is found. The reflection coefficient determines a compactly supported $q(x)$ uniquely [R9], but we give a new proof. If $q(x)$ is compactly supported, then the reflection coefficient $r(k):=\frac{b(k)}{a(k)}$ is meromorphic. Therefore, its values for all $k>0$ determine uniquely $r(k)$ in the whole complex $k$ plane as a meromorphic function. The poles of this function in the upper half-plane are the numbers $i k_{j}, j=1,2, \ldots, J$. They determine uniquely the numbers $k_{j}, 1 \leq j \leq J$, which are a part of the standard scattering data $\left\{r(k), k_{j}, s_{j}, 1 \leq j \leq J\right\}$, where $s_{j}$ are the norming constants. Note that if $a\left(i k_{j}\right)=0$ then $b\left(i k_{j}\right) \neq 0$ : otherwise equation (11.1.6) would imply $f\left(x, i k_{j}\right) \equiv 0$ in contradiction to the first relation (11.1.3). If $r(k)$ is meromorphic, then the norming constants can be calculated by the formula $s_{j}=-i \frac{b\left(i k_{j}\right)}{\dot{a}\left(i k_{j}\right)}=-i \operatorname{Re} s_{k=i k_{j}} r(k)$, where the dot denotes differentiation with respect to $k$, and Res denotes the residue. So, for compactly supported potential the values of $r(k)$ for all $k>0$ determine uniquely the standard scattering data, that is, the reflection coefficient, the bound states $-k_{j}^{2}$ and the norming constants $s_{j}, 1 \leq j \leq J$. These data determine the potential uniquely. Theorem 11.1.3 is proved.

Lemma 11.1.4. If $J=0$ then ind $f=$ ind $g=0$.

Proof. We prove ind $f=0$. The proof of the equation $i n d g=0$ is similar. Since ind $f(k)$ equals to the number of zeros of $f(k)$ in $\mathbb{C}_{+}$, we have to prove that $f(k)$ does not vanish in $\mathbb{C}_{+}$. If $f(z)=0, z \in \mathbb{C}_{+}$, then $z=i k, k>0$, and $-k^{2}$ is an eigenvalue of the operator $\ell$ in $L^{2}(0, \infty)$ with the boundary condition $u(0)=0$.

From the variational principle one can find the negative eigenvalues of the operator $\ell$ in $L^{2}\left(\mathbb{R}_{+}\right)$ with the Dirichlet condition at $x=0$ as consequitive minima of the quadratic functional. The minimal eigenvalue is:

$$
-k^{2}=\inf \int_{0}^{\infty}\left[u^{\prime 2}+q(x) u^{2}\right] d x:=\kappa_{0}, \quad u \in \stackrel{\circ}{H^{1}}\left(\mathbb{R}_{+}\right), \quad\|u\|_{L^{2}\left(\mathbb{R}_{+}\right)}=1,
$$

where $\stackrel{\circ}{H}^{1}\left(\mathbb{R}_{+}\right)$is the Sobolev space of $H^{1}\left(\mathbb{R}_{+}\right)$-functions satisfying the condition $u(0)=0$.

On the other hand, if $J=0$, then

$$
0 \leq \inf \int_{-\infty}^{\infty}\left[u^{\prime 2}+q(x) u^{2}\right] d x:=\kappa_{1}, \quad u \in H^{1}(\mathbb{R}), \quad\|u\|_{L^{2}(\mathbb{R})}=1 .
$$

Since any element $u$ of $\stackrel{\circ}{H}^{1}\left(\mathbb{R}_{+}\right)$can be considered as an element of $H^{1}(\mathbb{R})$ if one extends $u$ to the whole axis by setting $u=0$ for $x<0$, it follows from the variational definitions (11.1.24) and (11.1.25) that $\kappa_{1} \leq \kappa_{0}$. Therefore, if $J=0$, then $\kappa_{1} \geq 0$ and therefore $\kappa_{0} \geq 0$. This means that the operator $\ell$ on $L^{2}\left(\mathbb{R}_{+}\right)$with the Dirichlet condition at $x=0$ has no negative eigenvalues. This means that $f(k)$ does not have zeros in $\mathbb{C}_{+}$, if $J=0$. Thus $J=0$ implies ind $f(k)=0$.

Lemma 11.1.4 is proved. 
Remark 11.1.5. The above argument shows that in general

$$
\text { ind } f \leq J \quad \text { and } \quad \text { ind } g \leq J,
$$

so that (11.1.23) implies

$$
\operatorname{ind} m(k) \geq 0 \text {. }
$$

Therefore the Riemann problem (11.1.19) is always solvable. 


\section{Bibliography}

[AR] De Alfaro, V., Regge, T., Potential Scattering, North Holland, Amsterdam, 1965.

[ARS] Airapetyan, R., Ramm, A. G., Smirnova, A., "Example of two different potentials which have practically the same fixed-energy phase shifts", Phys. Lett. A, 254 (3-4), (1999), 141-148.

[B] Borg, G. [1946] "Eine Umkehrung der Sturm-Liouvilleschen Eigenwertaufgabe", Acta Math., 78, N1, (1946), pp. 1-96.

[CS] Chadan K., Sabatier P., Inverse Problems in Quantum Scattering Theory, Springer, New York, 1989 .

[CT] Cox, J. and Thompson, K., "Note on the uniqueness of the solution of an equation of interest in inverse scattering problem", J. Math. Phys., 11 (3), (1970), 815-817.

[DT] Deift, P., Trubowitz, E., "Inverse scattering on the line", Comm. Pure Appl. Math., 32, (1979), 121-251.

[E] Evgrafov, M., Analytic functions, Saunders, Philadelphia, 1966.

[G] Gakhov, F., Boundary value problems, Pergamon Press, New York, 1996.

[Ge] Gel'fand, I., Levitan, B., "On the determination of a differential equation from its spectral function," Izvestiya Akad. Nauk SSSR. Ser. Mat. 15, (1951). 309-360. (in Russian)

[GR] Gradshteyn I., Ryzhik I., Table of integrals, series and products, Acad. Press, Boston, 1994.

[GRS] Gel'fand, I., Raikov, D., Shilov, G., Commutative normed rings, Chelsea, New York, 1964.

[GS] Gesztesy, F., Simon, B., "A new approach to inverse spectral theory II. General real potentials and the connection to the spectral measure", Ann. of Math. 152, (2000), 593-643.

[GS1] Gesztesy, F., Simon, B., "On local Borg-Marchenko uniqueness result", Comm. Math. Phys., 218, (2000), 273-287.

[K1] M.G. Krein, "Theory of accelerants and $S$-matrices of canonical differential systems", Doklady Acad. Sci. USSR, 111, N6, (1956), 1167-1170.

[K2] Topics in differential and integral equations and operator theory, Birkhäuser, Basel, 1983.

[Leb] Lebedev, N., Special functions and their applications, Dover, New York, 1972.

[Lev] Levin, B., Distribution of Zeros of Entire Functions, Amer. Math. Soc., Providence RI, 1980,

[L] Levitan, B., Inverse Sturm-Liouville problems, VNU Press, Utrecht, 1987. 

Generalized translation operators, Jerusalem, 1964.

[L2] - "On the completeness of the products of solutions of two Sturm-Liouville equations", Diff. and Integr. Eq., 7, N1, pp.1-14.

[M] V.A. Marchenko, Sturm-Liouville operators and applications, Birchäuser, Basel, 1986.

[MP] S.Mikhlin, S.Prösdorf, Singular integral operators, Springer Verlag, Berlin, 1986.

[Nai] Naimark, M.A., Linear differential operators, Frederick Ungar Publishing Co., New York, 1968.

[N] Newton R., Scattering Theory of Waves and Particles, Springer, New York, 1982.

[N2] _ "Construction of potentials from phase shifts at fixed energy", J. Math. Phys., 3, 1, (1962), 75-82.

[N3] - "Connection between complex angular momenta and the inverse scattering problem", J. Math. Phys., 8, 8, (1967), 1566-1570.

[Ra] Ramm, A. G., Random fields estimation theory, Longman Scientific and Wiley, New York, 1990.

[R] Multidimensional inverse scattering problems, Longman Scientific \& Wiley, New York, (1992). Expanded Russian edition, Mir, Moscow, (1994).

[R1] - "Conditions under which the scattering matrix is analytic", Soviet Phys. Doklady, 157, (1964), 1073-1075. Math. Rev. 32 \#2049.

[R2] - Symmetry properties for scattering amplitudes and applications to inverse problems, J. Math. Anal. Appl., 156, (1991), 333-340.

[R3] _ "An inverse problem for the heat equation", Proc.Roy.Soc. Edinburgh, 123, N6, (1993), 973-976.

[R4] - "Formula for the radius of the support of the potential in terms of the scattering data", (with J.H.Arredondo and B.G.Izquierdo) Jour. of Phys. A, 31, N1, (1998), L39-L44.

[R5] - "Recovery of compactly supported spherically symmetric potentials from the phase shift of s-wave", In the book: Spectral and scattering theory, Plenum Publishers, New York, 1998 (ed. A.G.Ramm), pp.111-130.

[R6] , "Recovery of a quarkonium system from experimental data", Jour. of Phys. A, 31, N15, (1998), L295-L299.

[R7] - "Recovery of the potential from fixed energy scattering data", Inverse Problems, 4, (1988), 877-886.

[R8] - "Compactly supported spherically symmetric potentials are uniquely determined by the phase shift of s-wave", Phys. Lett. A, 242, N4-5, (1998), 215-219.

[R9] - "Property C for ODE and applications to inverse problems", in the book Operator Theory and Its Applications, Amer. Math. Soc., Fields Institute Communications, Providence, RI, 25, (2000), 15-75.

[R10] _ "Inverse scattering with part of the fixed-energy phase shifts", Comm. Math. Phys., 207 (1), (1999), 231-247.

[R11] — "A counterexample to a uniqueness result", Applic. Anal., 81, N4, (2002), 833-836. 
[R12] _ "Analysis of the Newton-Sabatier scheme for inverting fixed-energy phase shifts", Applic. Analysis, 81, N4, (2002), 965-975.

[R13] _ "A new approach to the inverse scattering and spectral problems for the Sturm-Liouville equation", Ann. der Phys., 7, N4, (1998), 321-338.

[R14] _ "An approximate method for solving inverse scattering problem with fixed-energy data", Jour. of Inverse and Ill-Posed Problems, 7, N6, (1999), 561-571. (with W.Scheid)

[R15] _ "A numerical method for solving the inverse scattering problem with fixed-energy phase shifts", Jour. of Inverse and Ill-Posed Problems, 8, N3, (2000), 307-322. (with A.Smirnova)

[R16] — "Krein's method in inverse scattering", in the book Operator Theory and Its Applications, Amer. Math. Soc., Fields Institute Communications vol.25, pp.441-456, Providence, RI, 2000 .

[R17] — "New proof of Weyl's theorem", IJDEA (Intern. J of Diff. Eq. and Appl.), 3, N1, (2001), $31-37$.

[R18] — "Piecewise-constant positive potentials with practically the same fixed-energy phase shifts", Applicable Analysis, 78, N1-2, (2001), 207-217. (with S.Gutman)

[R19] _ "An inverse problem for the heat equation", Jour. of Math. Anal. Appl., 264, N2, (2001), 691-697.

[R20] - "Stability of solutions to inverse scattering problems with fixed-energy data", Milan Jour. of Math., 70, (2002), 97-161.

[R21] - "Stable identification of piecewise-constant potentials from fixed-energy phase shifts", Jour. of Inverse and Ill-Posed Probl., 10, N4, (2002), 345-360. (with S.Gutman)

[R22] — "An inverse problem for the heat equation II", Applic. Analysis, 81, N4, (2002), 929-937.

[R23] _ "Theory of ground-penetrating radars II", Jour. of Inverse and Ill-Posed Probl.,6, N6, (1998), 619-624.

[R24] _ "Reconstruction of the potential from I-function", Jour. of Inverse and Ill-Posed Probl., 10, N4, (2002), 385-395.

[R25] _ "A new approach to inverse spectral theory III". Short range potentials, J. d'Analyse Math., 80, (2000), 319-334. (with B.Simon)

[R26] _ "Property C for ODE and applications to inverse scattering", Zeit. für Angew. Analysis, 18, N2, (1999), 331-348.

[R27] _ "Inverse scattering by the stability index method", Jour. of Inverse and Ill-Posed Probl., 10, N5, (2002), 487-502. (with S.Gutman and W.Scheid)

[R28] — "Inverse scattering on half-line", J. Math. Anal. App. 133, 2, (1988), 543-572.

[R29] _ "Example of a potential in one-dimensional scattering problem for which there are infinitely many purely imaginary resonances", Phys. Lett. A. 124, (1987), 313-319. (with B.A. Taylor).

[RK] Ramm, A .G., Katsevich, A. I., The Radon Transform and Local Tomography, CRC Press, Boca Raton, 1996

[RS] Reed, M., Simon, B., Methods of modern mathematical physics, Acad. Press, New York, 1982. 
[TQR] H. Thacker, C. Quigg, J. Rosner, "Inverse scattering problem for quarkonium systems", Phys. Rev. D, 181 (1978), 274-295. 